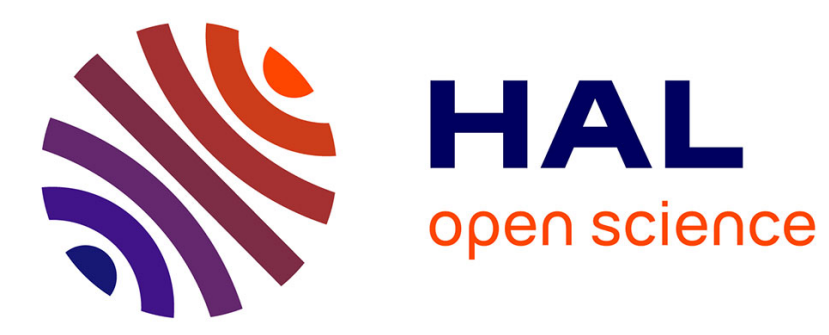

\title{
Les rapports communautaires aux espaces forestiers entre politiques du dehors et stratégies du dedans: les montagnes-forêts de Sasaguri
}

\author{
Anne Bouchy
}

\section{- To cite this version:}

Anne Bouchy. Les rapports communautaires aux espaces forestiers entre politiques du dehors et stratégies du dedans: les montagnes-forêts de Sasaguri. Cahiers d'Extrême-Asie, 2013, no. 22, p. 115-202. halshs-03134684

\section{HAL Id: halshs-03134684 \\ https://shs.hal.science/halshs-03134684}

Submitted on 8 Feb 2021

HAL is a multi-disciplinary open access archive for the deposit and dissemination of scientific research documents, whether they are published or not. The documents may come from teaching and research institutions in France or abroad, or from public or private research centers.
L'archive ouverte pluridisciplinaire HAL, est destinée au dépôt et à la diffusion de documents scientifiques de niveau recherche, publiés ou non, émanant des établissements d'enseignement et de recherche français ou étrangers, des laboratoires publics ou privés. 


\section{Les rapports communautaires aux espaces forestiers entre} politiques du dehors et stratégies du dedans : les montagnes-forêts de Sasaguri

Anne Bouchy

\section{Citer ce document / Cite this document :}

Bouchy Anne. Les rapports communautaires aux espaces forestiers entre politiques du dehors et stratégies du dedans : les montagnes-forêts de Sasaguri. In: Cahiers d'Extrême-Asie, vol. 22, 2013. Le vivre ensemble à Sasaguri, une communauté de Kyūshū. Dans l'entrelacs des dynamiques du dedans et du dehors. pp. 115-202;

doi : https://doi.org/10.3406/asie.2013.1417

https://www.persee.fr/doc/asie_0766-1177_2013_num_22_1_1417

Fichier pdf généré le 06/02/2019 


\begin{abstract}
In the context of the present research program, this essay focuses on the relation and interrelation between the local society of Sasaguri and the forest and mountain spaces which occupy more than 60 $\%$ of the municipality's surface. In examining how the modalities of this interrelation were constructed and continue to be constructed, the author attempts to stress the complex dynamics that presided over the transformations of the natural environment and the local community, in the middle of political, social and economic evolution on the national and international levels, that informed and overwhelmed its "internal" and "external" dimensions. This survey allows us also to better understand how and why forest in Sasaguri could be labelled as "nature" and "cultural heritage" at the same time.

For this purpose, the author first situates Sasaguri's land in the topographic and social context of the area, in order to show how it was open and prone to circulation. In a short presentation, she describes also the conceptual and disciplinary framework of Japanese ethnology and social anthropology, in which this approach takes its place. After having showed the diversity of the local vegetal environment, in the second section, she summarizes the usage of the term "forest-mountain" (sanrin), as folk resources which were overwhelmed by various policies and recent economical breakdowns, and points out the structural impact of the transformation of property systems. The main issues are at the same time the collective dispositions of managements, exploitation, and also the relation toward this "heritage" which is at the same time material, cultural and social, known as the "village-mountain" (sato-yama); and in a more general perspective, the eco-system constituted by these forested spaces. In the third section, several types of communal managements are analyzed, among which forest cooperatives, and the "forest of which the benefit is shared" (bunshū-rin) between several administrative and social entities. These analysis allow us to shed light on the modern evolution of the practice of "common lands" (iri.ai-chi) and their contemporary reevaluation, in local context as well as in the human sciences. The emblematic case of Mount Wakasugi and its village will let us integrate all these dynamics and to situate it in regard to the problem of the role of religious elements as a determinant operator in the relation of society with the forest. The national label of "therapy forest" that Sasaguri succeeded in obtaining in 2009 seems to be the most recent form of the interrelations around which the local identity is constructed.
\end{abstract}




\title{
LES RAPPORTS COMMUNAUTAIRES AUX ESPACES FORESTIERS ENTRE POLITIQUES DU DEHORS ET STRATÉGIES DU DEDANS : LES MONTAGNES-FORÊTS DE SASAGURI
}

\author{
Anne Bouchy
}

In the context of the present research program, this essay focuses on the relation and interrelation between the local society of Sasaguri and the forest and mountain spaces which occupy more than $60 \%$ of the municipality's surface. In examining how the modalities of this interrelation were constructed and continue to be constructed, the author attempts to stress the complex dynamics that presided over the transformations of the natural environment and the local community, in the middle of political, social and economic evolution on the national and international levels, that informed and overwhelmed its "internal" and "external" dimensions. This survey allows us also to better understand bow and why forest in Sasaguri could be labelled as "nature" and "cultural beritage" at the same time.

For this purpose, the author first situates Sasaguri's land in the topographic and social context of the area, in order to show how it was open and prone to circulation. In a short presentation, she describes also the conceptual and disciplinary framework of Japanese ethnology and social antbropology, in which this approach takes its place. After baving showed the diversity of the local vegetal environment, in the second section, she summarizes the usage of the term "forest-mountain" (sanrin 山林), as folk resources which were overwhelmed by various policies and recent economical breakdowns, and points out the structural impact of the transformation of property systems. The main issues are at the same time the collective dispositions of managements, exploitation, and also the relation toward this "beritage" which is at the same time material, cultural and social, known as the "village-mountain" (sato-yama 里山); and in a more general perspective, the eco-system constituted by these forested spaces. In the third section, several types of communal managements are analyzed, among which forest cooperatives, and the "forest of which the benefit is shared" (bunshū-rin 分収林) between several administrative and social entities. These analysis allow us to shed light on the modern evolution of the practice of "common lands" (iri.ai-chi 入会地) and their contemporary reevaluation, in local context as well as in the buman sciences. The emblematic case of Mount Wakasugi 若杉山 and its village will let us integrate all these dynamics and to situate it in regard to the problem of the role of religious elements as a determinant operator in the relation of society with the forest. The national label of "therapy forest" that Sasaguri succeeded in obtaining in 2009 seems to be the most recent form of the interrelations around which the local identity is constructed. 
Thus the distinction between environment and nature corresponds to the difference in perspective between seeing ourselves as beings within a world and as beings without it.

Tim Ingold

The Perception of the Environment ${ }^{1}$

En 2009, la commune de Sasaguri 簙栗町 a acquis le label national de «Thérapie forestière » (Shinrin Serapī 森林セラピー), par lequel est officiellement reconnue aux denses forêts communales leur qualité " thérapeutique ». L'espace forestier est désormais proposé aux visiteurs et aux touristes comme lieu privilégié pour des 《bains de forêts » (shinrin yoku 森林浴), reconnus bénéfiques dans la lutte contre les effets néfastes du stress citadin. Pourtant, il y a presque trois cents ans, en 1737 , affligé par l'état de déforestation des montagnes « sur lesquelles ne se dressaient plus que quelques vieux cryptomères épars ${ }^{2}$, Nagahama Shichirō Dayū 長浜七郎 太夫, guerrier résidant à Gō-no-haru 郷之原, utilisa ses propres fonds pour planter 65200 cryptomères et pins. Entreprise de reforestation qui, comme nous le verrons, fut continuée trente ans plus tard sur une plus vaste échelle. Aujourd'hui, les pentes des montagnes communales sont en grande partie plantées, et le cryptomère (Cryptomeria japonica, sugi 杉, 椙) est devenu l'arbre symbole de la commune. Mais peu d'habitants savent encore que l'apparence de leur milieu de vie a changé du tout au tout au cours des dernières huit ou neuf générations de leurs familles. Il leur est difficile d'imaginer un paysage qui ne soit pas coloré du vert profond des cryptomères, alors qu'ils habitent au pied du « mont du Jeune cryptomère » (Wakasugi yama 若杉山) $)^{3}$. Vu du ciel, l'espace communal de Sasaguri est aujourd'hui en effet majoritairement recouvert de forêts (fig. I). Mais, sous cette uniformité verte se cache une grande variété de réalités, non seulement des peuplements sylvestres, mais aussi des relations - matérielles, sociales, administratives, économiques et symboliques - à ces forêts et à ces bois.

La fluctuation du rapport de la société locale au milieu forestier est l'angle d'approche de ma contribution à ce programme de recherche collectif, qui se propose d'examiner les dynamiques traversant et reliant ce qui est pensé et donné comme l'« intérieur » et l'« extérieur » des groupes sociaux constitutifs de la commune de Sasaguri $i^{4}$. Mon travail s'inscrit dans une réflexion portant sur les modalités de la relation communautaire à l'environnement dans la longue durée. Cette démarche

I. Tim Ingold, The Perception of the Environment: Essays on Livelibood, Dwelling and Skill, 2000 , p. 20.

2. Sasagurichō bunkazai senmon iinkai 篠栗町文化財尃門委員会 (éd.), Sasaguri chōshi. Rekishiben 篠栗町誌: 歴史編, 1982, p. 220 (abrégé ci-après en Sasaguri chōshi).

3. Sur l'origine de ce toponyme, voir dans ce même volume Suzuki Masataka 鈴木正崇, "Continuités et transformations de la société locale. Le fait coutumier dans le village de Wakasugi ", p. 52.

4. Voir, au début du volume, la présentation de ce programme et de son déroulement, Anne Bouchy, "Sasaguri. Dedans, dehors. Une approche ethnologique collective », p. I-9. 


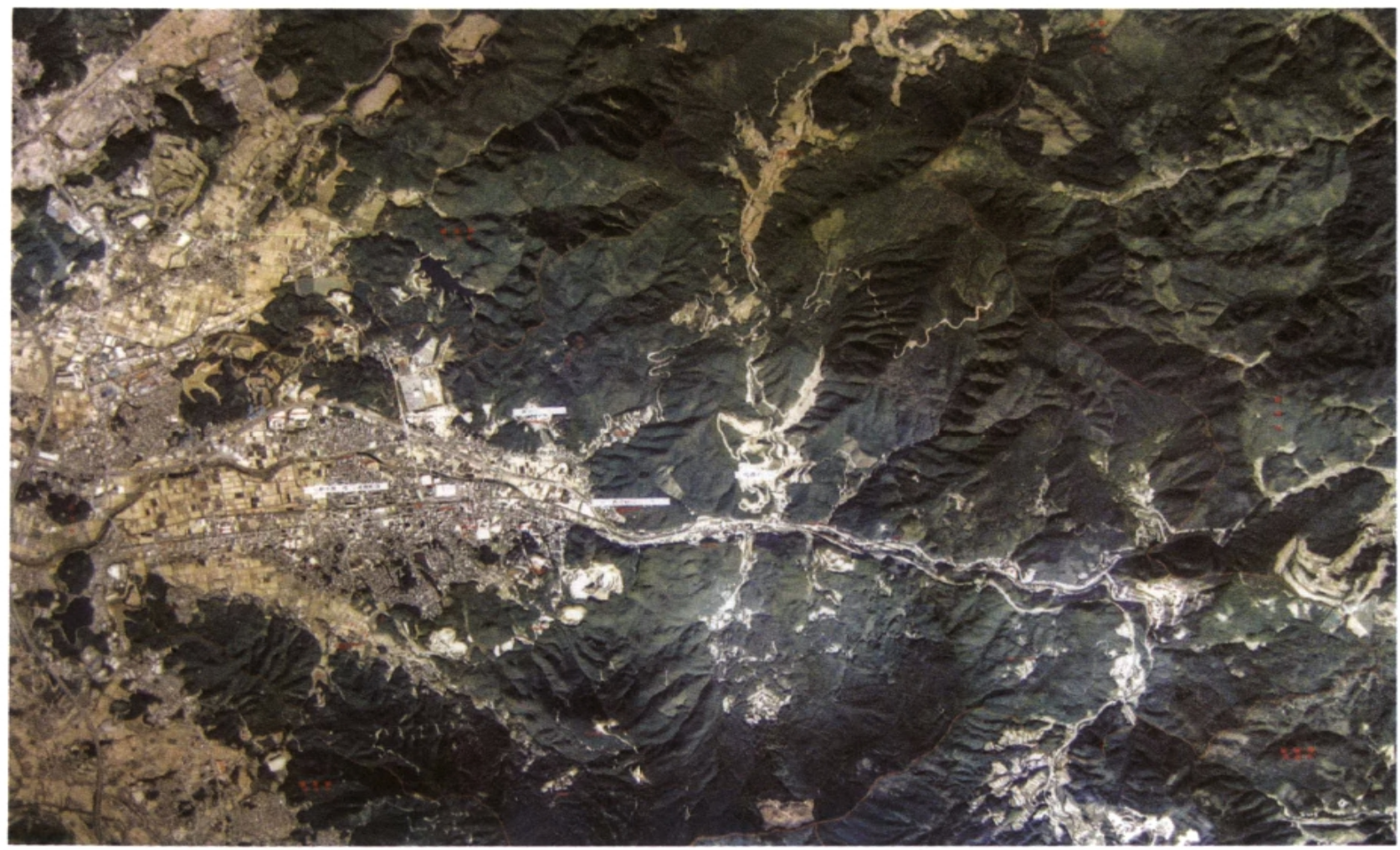

Fig. I : Vue aérienne de Sasaguri.

(Centre de documents historiques et de matériaux ethnographiques de Sasaguri.)

combine une investigation sur les réalités historiques et contemporaines du milieu naturel, et un questionnement sur les contraintes pesant sur les divers modes d'action adoptés pour agir sur ce milieu naturel ${ }^{5}$, telles qu'elles sont imposées à la fois par cet environnement et par le contexte social. L'ensemble de ces données permet de faire apparaître un certain nombre de caractéristiques des modalités et des effets physiques, sociaux et culturels de cette complexe interaction.

L'objectif de mon propos est pluriel. Il est d'abord de mettre en lumière, par le biais de l'exemple de Sasaguri, la façon dont, à travers le temps, les imbrications et les fluctuations politiques, sociales, économiques formatent et bouleversent les dimensions « internes » et « externes » d'une communauté locale, dont les « traditions » et les entités collectives se révèlent fluides, osmotiques et sans cesse modifiées, par-delà même les discours de fixation et de continuité. Les réalités historiques et actuelles nous offrent ainsi des éléments pour proposer une alternative au, ou un

5. J'utilise le terme milieu pour désigner la physicalité du monde, et celui d'environnement, la relation qu'une société et sa culture entretiennent avec un milieu donné (cf. les travaux de Patrick Pérez, par exemple : "Elements of an American Landscape: The Arizona Hopi ", dans Arnar Àrnason et al., éd., Landscapes beyond Land: New Ethnographies of Landscape and Environment, 2012, p. 83-97 ; et "Ce que les Hopi m’ont appris sur le paysage », Les Annales de Géographie, 691 (juin 2013), p. 243-265). 


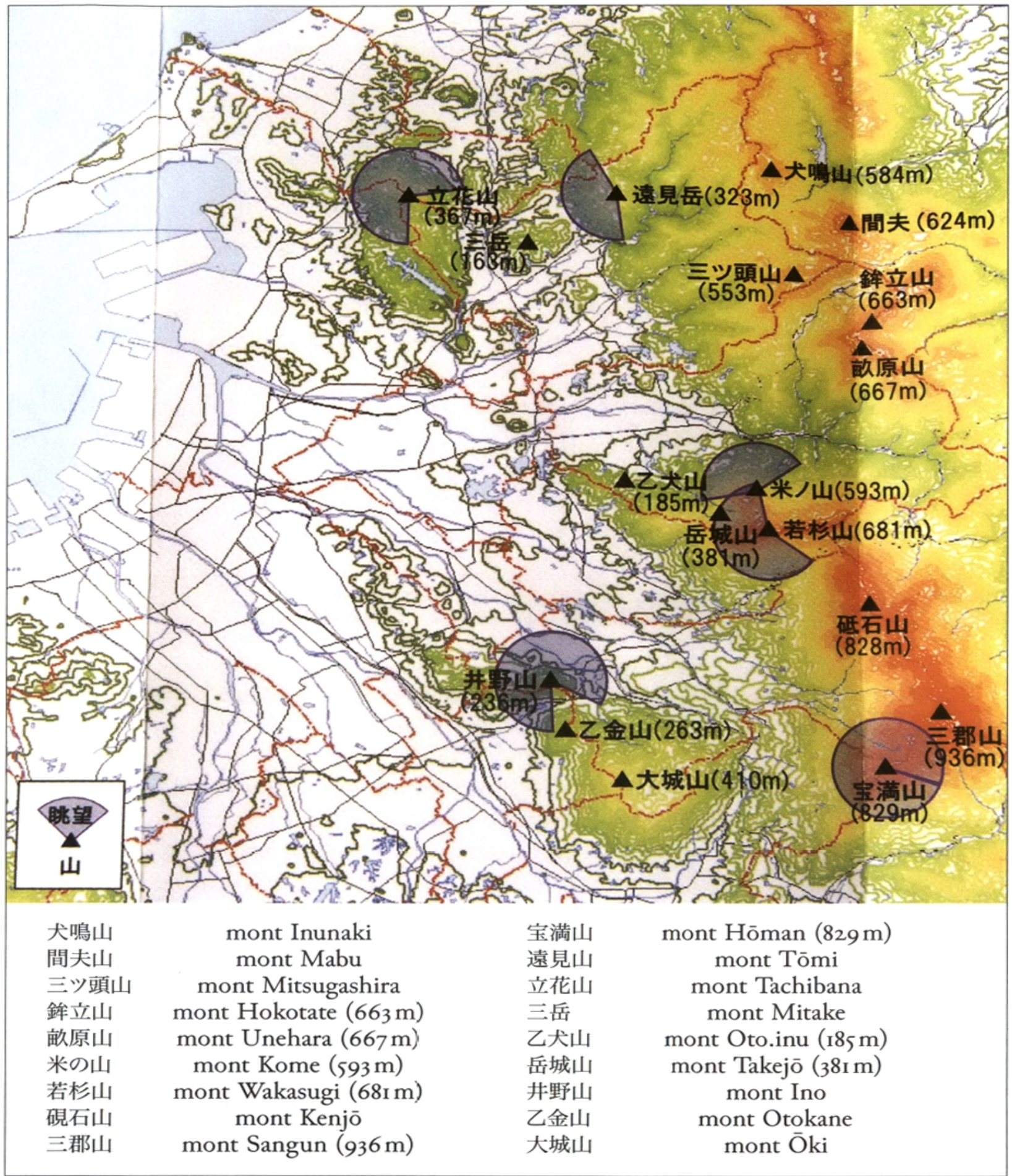

Carte I : Le massif montagneux de Sangun. (D'après une carte de la brochure Kasuya no midori かすやの緑 (Kasuya vert), édité par le canton de Kasuya). 
dépassement du modèle statique et dualiste « intérieur-extérieur » souvent privilégié par les études des spécialistes japonais de l'ethnologie du Japon.

Ensuite, il s'agit également de contribuer à une réflexion sur la question, aujourd'hui plus que jamais d'actualité, des rapports de la société, des humains, au milieu naturel, et par là de réfléchir à la construction de la notion de " nature ». Sur ce sujet, la société japonaise offre des éléments originaux et parfois dérangeants. En effet, tout en étant héritière d'une conception ancienne du monde selon laquelle l'opposition nature-culture n'est pas pertinente et toujours vivante de nos jours, elle a également intégré cette opposition sous l'influence des sciences occidentales à partir de la fin du XIX siècle. Ceci est à l'origine d'un entremêlement (riche mais perturbateur) des registres conceptuel et linguistique reflété dans les représentations, les modèles et les modalités d'action. Ce travail est donc aussi une tentative pour apporter quelques clés de décryptage permettant d'entrevoir ce qui sous-tend souvent l'ambiguiité des discours comme des politiques publiques et des comportements individuels contemporains, notamment sur les questions d'environnement.

\section{Cadre général de l'étude : les espaces montagneux et forestiers dans le contexte local et national, et dans les études d'ethnologie du Japon}

\section{Sasaguri : territoire fermé et ouvert}

Née en I955 (Shōwa 昭和 30) de la fusion administrative du village de Seto 勢門村 et de la commune de Sasaguri (eux-mêmes constitués en 1889, Meiji 明治 22, de la réunion de six villages pour le premier et de quatre pour la seconde ${ }^{6}$ ), la commune de Sasaguri, située au centre du département de Fukuoka, s'étend sur une superficie de $38,90 \mathrm{~km}^{2}$ (E-O : $8 \mathrm{~km}, \mathrm{~N}-\mathrm{S}: 7 \mathrm{~km}$ ) (carte I de la présentation, p. IO) ${ }^{7}$. Géographiquement, elle constitue la partie médiane du côté occidental du massif montagneux entièrement boisé de Sangun 三郡山地 (« des Trois cantons $»^{8}$ - carte I), où se rejoignent les frontières des trois cantons de Kasuya 糟屋郡, Chikushi 筑紫郡 et Kaho 嘉穂郡. Le territoire communal a la forme d'un éventail ouvert, orienté est-ouest, dont le bord large se relève au nord avec le sommet du mont Mitsugashira 三ツ頭山 (« Trois têtes », $553 \mathrm{~m}$ ), à l'est avec les monts Hokotate 鉾立山 (《Dresse-hallebarde », 663,2 m) et Unebaru 畧原山 (« du Champ de sillons », $667 \mathrm{~m}$ ), au sud avec les monts Wakasugi

6. En I889, les quatre villages de Sasaguri 篠栗, Kana.ide 金出, Takata 高田 et Haginoo 秋尾 se regroupent pour former le village de Sasaguri 筗栗村, et les six villages d'Onaka 尾仲, Wakasugi 若杉, Oto.inu 乙犬, Wada 和田, Tsubakuro 津波黒 et Tanaka 田中 se regroupent pour constituer celui de Seto 勢門村. Cette réorganisation commencée dès Meiji 6 (1873) fut imposée par une succession de mesures et contre-mesures nationales, par lesquelles le gouvernement cherchait à affirmer sa volonté de changement social, politique et administratif. Le nom des anciens villages est celui des quartiers $(k u$ 区) actuels.

7. Voir les précisions dans l'introduction générale du présent volume, cf. Anne Bouchy, "Sasaguri. Dedans, dehors ", p. 9-14.

8. Le plus haut sommet de ce massif montagneux est l'éponyme mont Sangun - - 郡山 (des Trois cantons), 936 mètres d'altitude, situé au sud-est de Sasaguri. 
若杉山 («Jeune cryptomère », 681 m) et Kome 米の山 (« du Riz », 593,7 m), et dont la poignée correspond à l'extrémité occidentale qui s'abaisse en une plaine communiquant avec les arrondissements urbanisés de l'est de Fukuoka 福岡, métropole de Kyūshū (Higashi-ku 東区 et Hakata-ku 博多区), et les communes de Kasuya 粕谷町 et Hisayama 久山町 (carte 2 de la présentation, p. 12). Cet éventail est traversé dans sa partie médiane par la rivière Tatara (多々良川, $17,4 \mathrm{~km}$ ) qui prend sa source à Naijū 内住峡, au fond des montagnes du sud-est de la commune, et se jette à l'ouest dans la baie de Hakata 博多湾. La partie orientale de la commune (ancien village de Seto) a un double caractère. C'est un petit bassin qui est la principale zone agricole/rizicole, vers laquelle converge l'abondante eau des montagnes qui l'entourent sur trois côtés, et c'est le centre extrêmement urbanisé de la municipalité. Ces deux aspects résultent d'une occupation très ancienne des lieux confirmée par l'archéologie'. Les autres espaces habités sont concentrés le long de la vallée transversale de la rivière Tatara et dans les dix vallées à plus ou moins fortes pentes qui descendent vers elle. Tout le reste de la commune est majoritairement montagneux et forestier, avec de petites extensions agricoles au cœur des zones boisées des extrémités sud, vallée de Wakasugi, nord-est, Haginoo 萩尾, et sud-est, Naijū 内住, de cet éventail. Notons d'emblée que montagnes et collines coïncident topographiquement avec l'espace forestier.

L'utilisation des sols sur les 3890 hectares de la commune est la suivante ${ }^{10}$ :

- superficie totale de l'espace de montagnes-forêts : 2395 ha soit $61,56 \%$ du territoire

communal ;

- espaces non habités : 2395 ha $(61,56 \%)$;

- surfaces agricoles : 265 ha (rizières 227 ha, champs 38 ha) $(6 \%)^{11}$;

- superficie totale des espaces habités : 333 ha $(8,6 \%)$;

- voies terrestres : 199 ha $(5,1 \%)$;

- surfaces aquatiques : 90, I ha (fleuve, rivières, étangs, retenues d'eau) $(2,3 \%)$.

Premiers constats : la commune, en tant qu'entité administrative, est une formation récente composée d'une mosaïque d'anciens villages réunis (en six étapes à Meiji, puis au xxe siècle dans la forme actuelle), du fait de leur proximité géographique, pour des questions politiques et institutionnelles; l'espace communal est caractérisé par un fort contraste résultant, d'une part, d'une concentration de

9. Les plus anciens vestiges remontent aux périodes Jōmon 縄文 (de 14000 ans à I O0o ans avant notre ère), Yayoi 弥生 (après la période Jōmon jusque vers III ${ }^{e}$ siècle), Kofun 古墳 (après la période Yayoi jusque vers la fin du vil' siècle) et sont regroupés dans cette partie ouest autour de la rivière (Sasaguri chōshi, p. 7-40). Le toponyme Seto 勢門 (en man.yō gana : 世歫) apparaît dans le premier dictionnaire encyclopédique en langue japonaise Wamyō ruijushō 和名類聚抄 (938) dans la liste des neuf villages du canton de Kasuya du pays de Chikuzen 筑前 (ancien nom de la région correspondant à la partie nord-ouest de l'actuel département de Fukuoka).

10. D’après les chiffres publiés en 2008 par la municipalité dans Sasaguri-chō kikaku kōhōka 篗栗町企画広報課, éd., Green Oasis Sasaguri, 2008-12 Dai yoji Sasaguri-chō sōgō keikaku. Köki kihon $k e i k a k u$ 第 4 次策栗町総合計画, 後期基本計画.

II. Ces surfaces agricoles ne cessent aujourd'hui de diminuer : 1992:313 ha, dont rizières 26I ha, champs 52 ha (Sasaguri-chō kikaku kōhōka 篠栗町企画広報課, éd., Green Oasis Sasaguri. 2003 Dai niji Sasaguri-chō kokudo riyō keikaku 第二次篠栗町国土利用計画, p. 5). 
la population dans l'espace limité du bassin occidental (où réside la majorité des 31532 habitants - chiffre de 2012) et d'autre part, de l'importance des zones montagneuses forestières, non habitées ou à habitat dispersé et peu dense, qui constituent la majorité du territoire communal.

Comme la topographie le laisse supposer, le côté occidental de Sasaguri est bien la « porte $»^{12}$ des échanges que ces communautés locales ont entretenus tout au long de l'histoire avec les centres urbains de Fukuoka et Hakata. Aujourd'hui, cette partie de Sasaguri est de fait l'une des villes-dortoirs (beddo-taun ベッドタウン, «bed-town ) satellites de Fukuoka, où habite mais ne travaille pas, une partie non négligeable de la population de la commune. À cette extrémité ouest s'entrecroisent toutes les grandes voies de circulation; d'ouest en est : la nationale 20I, la départementale 607 et la ligne de chemin de fer de Sasaguri, avec la gare centrale de Hakata à is minutes de train de celle de Sasaguri ; et du nord au sud : l'autoroute de Kyūshū avec sa sortie vers Fukuoka, les départementales 35 et 24, et la ligne de chemin de fer de Kashii 香椎線, sans oublier l'aéroport international de Fukuoka à vingt minutes du centre de Sasaguri par la départementale 607.

Cette entrée pourrait sembler un goulot d'étranglement, au-delà duquel, on butte au nord, au sud et à l'est, contre un espace montagneux qui, par ses pentes raides et ses vallées étroites, paraît en fermer les trois autres côtés et faire ainsi obstacle à toute circulation. Mais contrairement aux apparences, tel n'est pas le cas. Il est vrai que le massif des Trois cantons, Sangun sanchi 三郡山地, dans son ensemble, constitue bien une barrière entre la bordure côtière, la baie de Fukuoka à l'ouest, d'une part, et le nord et l'est de Kyūshū, d'autre part, c'est-à-dire l'accès à la mer intérieure, à Shikoku et à Honshū. Espace difficile d'accès, les vallées et forêts reculées de ce massif des Trois cantons ont de fait représenté un lieu favorable pour ceux qui, au cours des sic̀clcs, fuyant ennemis ou poursuites de toutes sortes, cherchaient un refuge hors d'atteinte. Ainsi, la légende locale (que l'on retrouve en de nombreux autres lieux du Japon) veut que la Roche Heike 平家岩, à côté de l'oratoire de Fudō 不動堂 $\left(\mathrm{n}^{\circ} 45\right)^{13}$ de Kido 城戸 soit l'endroit où, au Moyen Âge (fin du XII siècle), se cachèrent et s'implantèrent les guerriers Heike vaincus par les Genji 源氏 ${ }^{14}$. Néanmoins, cette inaccessibilité est aussi la raison pour laquelle les autorités locales n'ont eu de cesse d'ouvrir des passages permettant de traverser ce massif montagneux.

12. Seto 勢門, écrit aussi avec les caractères 迫門 au Moyen Âge, c'est-à-dire la " porte étroite ", désigne le passage terrestre et fluvial ouvert entre l'est et l'ouest, à l'endroit où se resserrent les montagnes, et dont le mont Iimori 飯盛山 (du Riz entassé, $356 \mathrm{~m}$ ) au nord et le mont de la Citadelle (Jōyama 城山 ou Takejō yama 岳城山, de la Citadelle de montagne, 381 m) au sud seraient les deux montants du portail (Aoyagi Tanenobu 青柳種信 (éd.), Chikuzen no kuni zoku fudoki shūi 筑前国 続風土記拾遺, 1993 ( $\mathrm{r}^{\mathrm{re}}$ éd. 1814-64), p. 40r. Sasaguri chöshi, p. 6r).

13. Les numéros entre parenthèses après le nom d'un temple ou d'un oratoire correspondent au numéro que ceux-ci portent dans le circuit de pèlerinage de Sasaguri.

14. Voir dans ce même volume Nakayama Kazuhisa 中山和久, «La dynamique de création, réplication et déclin des lieux de pèlerinage : le nouveau pèlerinage de Shikoku à Sasaguri ", p. 291-293. 


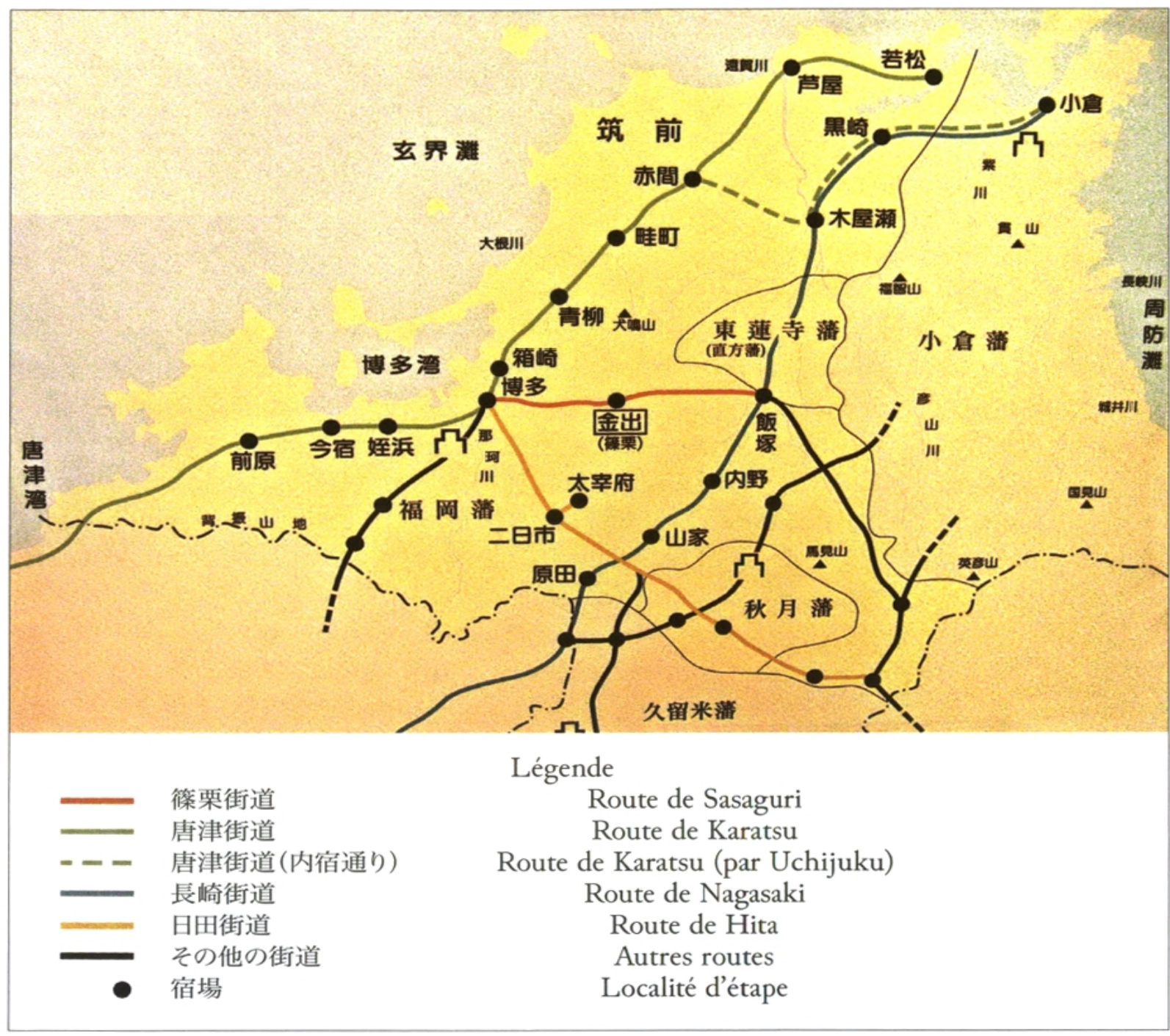

若松

小倉

筑前

玄界灘

福智山

博多湾

箱先

博多

東葉寺藩

Wakamatsu
Kokura
Chikuzen
mer Genkai
mont Fukuchi
baie de Hakata
Hakosaki
Hakata
fief de Tōren-ji

小倉藩
金出(篠栗)
飯塚
太幸府
唐津湾
福岡藩
秋月潘
英彦山
久留米藩

fief de Kokura

Kana.ide (Sasaguri)

Iizuka

Dazaifu

baie de Karatsu

fief de Fukuoka

fief d'Akizuki

mont Hiko

fief de Kurume

Carte 2 : Voies de circulation du nord-ouest de Kyūshū à l'époque d'Edo

(Panneau de la salle d'exposition du Centre de documents historiques et de matériaux ethnographiques de Sasaguri). (Ne sont indiqués que les principaux toponymes et ceux qui apparaissent dans les textes du volume.) 
L'extrémité escarpée de la vallée orientale, dite «du ou des yamabushi 山伏 $»^{15}$, a été percée dès le début du XvII ${ }^{e}$ siècle pour permettre les allées et venues entre l'ouest et l'est du fief de Fukuoka (ou de Chikuzen), dit aussi fief des Kuroda 黒田藩, du nom des daimyō 大名 qui le gouvernèrent tout au long d'Edo (de I600 à 1871). Cette voie de circulation appelée Route de Sasaguri, Sasaguri kaidō 筗栗街道, était l'un des six grands axes de circulation du fief (carte 2). Par Iizuka 飯塚, elle reliait directement la ville-sous-le-château (jōka-machi 城下町) de Fukuoka et la route côtière occidentale à la grande voie intérieure nord-sud qui, en passant par Kokura 小倉, gagnait Honshū. Sa position médiane sur le Sasaguri kaidō valut à Sasaguri d'en devenir la localité-étape (sbukuba machi 缩場町) officielle, avec ses structures d'hébergement et d'accueil des voyageurs. Le daimyó y construisit une résidence secondaire. Il empruntait cette route, notamment lors des allers et retours bisannuels entre Fukuoka et Edo pour aller rendre ses devoirs au shōgun 将軍. Le passage de montagne de cette route à Kido était périlleux et ne cessa d'être amélioré. En 189I, la route fut élargie, et tout au long des Sept tournants de la montée furent plantés mille cerisiers pour évoquer le célèbre paysage des mille cerisiers de Yoshino 吉野山 du département de Nara 奈良県. Ce lieu devint ainsi un espace touristique qui, depuis, attire les citadins de Fukuoka et du nord-est du département au moment de la floraison. Devenue route départementale en 1945, elle fut agrandie en 1947, puis devint une nationale (N 201) en 1968. En 1990, elle fut doublée d'une déviation qui traverse le massif montagneux par un tunnel (carte 2 de la présentation, p. 12). Le trafic routier global est évalué aujourd'hui à plus de quarante mille véhicules par jour ${ }^{16}$. Par ailleurs, l'ouverture en 1904 (Meiji 37) de la ligne de chemin de fer de Sasaguri 䉓栗線, ouverture concomitante de l'essor des mines de charbon locales, intensifia le trafic côté Fukuoka. En outre, en 1968, suite au percement du tunnel de Sasaguri sous cet escarpement de Kido, la voie ferrée, doublant la route, rejoignit à son tour le côté oriental du massif des Trois cantons ${ }^{17}$. Toute cette circulation séculière fut augmentée d'une dimension religieuse à partir du début du xix ${ }^{e}$ siècle, avec la création puis la vogue du nouveau pèlerinage de Shikoku dans la commune, vogue qui s'est prolongée jusqu'au XXI ${ }^{\mathrm{e}}$ siècle $^{18}$. Des foules de pèlerins venus de l'extérieur se

15. La légende veut que les lieux soient hantés par l'âme d'un yamabushi (pratiquant shugen; voir ci-après note 19) qui y aurait été tué par des bandits voulant lui prendre l'or qu'il transportait. Mais c'est aussi le lieu où, jusqu'à l'époque contemporaine, tous les yamabushi participant à l'entrée dans la montagne de printemps du groupe shugen 修験 du mont Höman 宝满|| stationnaient pendant sept jours, et où se trouvaient les tombes des yamabushi morts pendant ces pratiques (Mori Hiroko 森弘子, Höman-zan no kankyō rekishi gaku teki kenkyū 宝満山の環境歴史学的研究, 2008, p. 548).

16. En $2010: 35235$ véhicules par vingt-quatre heures sur la nationale 201. Sur la départementale, 13480 véhicules par vingt-quatre heures (2005). Chiffres communiqués en 2012 par les bureaux des routes nationales et départementales (Recensement du trafic routier de 2010). Ces évaluations ne tiennent pas compte du trafic sur les routes secondaires.

17. Aujourd'hui le nombre des voyageurs utilisant quotidiennement la gare de Sasaguri s'élève environ à 8000 (article du Nishi Nibon shinbun 西日本新聞 du 04/04/2008). Tous ces chiffres (routiers et ferroviaires) ne concernent que le trafic ordinaire, et doivent donc être largement majorés en période de pèlerinage à Sasaguri.

18. Voir la contribution de Nakayama Kazuhisa, "La dynamique de création, réplication et déclin des lieux de pèlerinage ", dans ce volume, p. 274-276. 
sont mises à parcourir à pied, puis en voiture ou en car, les quarante-six kilomètres de son circuit, qui pour l'essentiel se situe au cœur des forêts de Sasaguri.

Ainsi, loin d'être un cul de sac, la partie montagneuse et forestière orientale, Kido et Naijū, est un couloir de circulation dense qui relie la commune à l'extérieur sur l'axe est-ouest. Vers le nord et Munakata 宗像, l'autre axe de circulation important suit la vallée qui part de Kana.ide et traverse Haginoo et Nomiyama 呑山. Seul le côté sud, avec la barrière du mont Wakasugi, reste aujourd'hui non franchi par les voies routières ou ferroviaires. Depuis les années 1930, une route d'abord forestière, aujourd'hui asphaltée, accède au flanc nord de la montagne depuis Sasaguri, mais ne va pas jusqu'au sommet. Cependant, telle est la situation relative aux échanges avec l'extérieur sur de moyennes et longues distances, et ce, notamment, pour l'époque contemporaine. Il en va autrement en ce qui concerne les relations avec les localités limitrophes, en particulier à une époque un peu plus ancienne.

\section{Communautés par-delà les montagnes}

Les montagnes-forêts sont en effet perçues différemment selon les époques et notamment dès qu'elles sont envisagées, non plus seulement comme un cadre topographiquement et administrativement délimité, mais comme un espace de vie soustendant et sous-tendu par de multiples dynamiques socioculturelles (les activités de subsistance, le religieux, les échanges matrimoniaux). Ainsi, sous l'angle du religieux et du politique, le mont Wakasugi, siège d'une importante communauté bouddhique tendai 天台 et shingon 真言 ainsi que shugen ${ }^{19}$ dès l'Antiquitée ${ }^{20}$, loin de représenter

19. Shugen est utilisé d'une part comme nom commun et équivalent du terme yamabushi («celui qui couche dans la montagne ") pour désigner un adepte du shugendō ou l'une de ses organisations (ainsi, shugen du mont Hōman), et d'autre part comme adjectif. Dans ce dernier cas seulement il est en italiques. Shugendō : « voie (de l'acquisition) des pouvoirs par l'ascèse ", l'ascèse étant prise ici dans le sens premier d'ascesis, c'est-à-dire la pratique, l'entraînement pour acquérir la maîtrise d'un art, d'une technique, etc. Récemment les chercheurs utilisent fréquemment shugen à la place de shugendō. Le shugendō est l'un des courants historiques du fait religieux japonais. Né dans l'Antiquité au Japon de la fusion de croyances autochtones centrées sur les divinités des montagnes, de pratiques qui leur étaient associées, et d'éléments du bouddhisme et du taoïsme venus de Chine, il est originellement constitué de lignées initiatiques. Il s'est structuré progressivement en organisations, d'une part, territoriales, et, d'autre part, centralisées autour de deux pôles principaux formant les deux branches shugen majeures, qui sont le Honzan 本山派 (ayant à sa tête le Shōgo-in 聖護院, affilié au Tendai) et le Tōzan 当山派 (ayant à sa tête le Daigo-ji Sanbō-in 醍醐寺 deux situés à Kyōto. Forme cultuelle ayant fait fusionner et combinant quantités d'éléments de divers courants religieux, dont le bouddhisme et le shintō, il fut objet d'une interdiction en I872, suite à la Restauration de Meiji qui imposa la séparation des cultes shintō et bouddhiques. Néanmoins, il s'est perpétué jusqu'à nos jours, notamment après sa réorganisation officielle rendue possible par la garantie de la liberté des cultes et des pratiques religieuses instaurée par la constitution de 1946. L'histoire et les multiples dimensions du shugendō n'ont été progressivement mises au jour qu'à partir de la seconde moitié du $\mathrm{xx}^{e}$ siècle par les historiens et les ethnologues japonais.

2o. Voir dans ce même volume Suzuki Masataka, "Continuités et transformations de la société locale ", p. 34-36, 45-58, et Mori Hiroko, "Le mont Wakasugi, son sanctuaire et le shugen dans l'histoire de Kyūshū et de l'Asie de l'Est ", p. 209-215. 
un obstacle, a toujours été conçu comme un ensemble, dont le centre - pôle cultuel de toute la région jusqu'à l'époque moderne - est le sanctuaire situé à son sommet, Taiso gongen 太祖権現 ou Taiso-gū 太祖宮, soutenu par les diverses structures religieuses situées sur ses deux côtés. Le côté nord, dit "vallée de Droite " (Utani 右谷) était organisé autour du Kenshō-ji 建正寺 Seinan-in 西南院 (ermitage du Sud-ouest), et le côté sud, dit originellement «vallée de Gauche » (Satani 左谷 aujourd'hui 佐谷) autour du Sekisen(sui)-ji 石泉 (水) 寺 Tōhoku-in 東北院 (ermitage du nord-est ${ }^{21}$. Le côté septentrional fait partie du village de Wakasugi, composante actuelle de la commune de Sasaguri. Le côté méridional se trouve dans la commune de Sue 須恵町. Au Moyen Âge, cet ensemble religieux compta jusqu'à trois cents ermitages sur ces deux flancs montagneux, disparus dans les incendies des guerres médiévales locales. Néanmoins, par son affiliation au shugen du mont Hōman, et par l'importance stratégique que la montagne remplissait dans les pratiques d'entrée dans la montagne (mine.iri 峰入り ou nyübu 入峰), ce pôle shugen du mont Wakasugi fut entretenu jusqu’à Meiji par la maison des Ishii (dite ermitage Ishii 石井坊) de Wakasugi, où ceux-ci résident encore aujourd'hui. Les Ishii sont les descendants d'un yamabushi qui avait été délégué par le groupe shugen du mont Hōman au début d'Edo, après les destructions médiévales, pour assurer la continuité de la gestion du sanctuaire en tant que bettō (別当 administrateur) et l'entretien des chemins de montagnes. En effet, Satani et le mont Wakasugi étaient des étapes cruciales du deuxième jour d'un parcours rituel dit "sommet du printemps ( $3^{\mathrm{c}}$ et $4^{\mathrm{e}}$ mois) » (haru no mine 春の峯) de ce groupe shugen. Ce rite initiatique, appelé « entrée dans la montagne » et commun à toutes les branches du shugendō 修験道, a lieu annuellement sur le modèle d'une dramaturgie de la mort et de la renaissance rituelles.

Au cours de l'époque d'Edo, ce parcours rituel passant par le mont Wakasugi était organisé de façon grandiose par le shugen du mont Hōman pour l'intronisation de son nouveau dignitaire (zasu 座主). Pour ce dernier, c'était un événement capital, qui fut consigné par les acteurs eux-mêmes, et dont les traces écrites sont ainsi parvenues jusqu’à nous ${ }^{22}$. Mais, selon le supérieur du temple Hōzan-ji $\left(n^{\circ}{ }^{3} 4\right)$ qui domine cette vallée, il semble qu'indépendamment de cette entrée dans la montagne cérémonielle qui avait lieu tous les 20 ou 30 ans, il en y avait également d'autres, annuelles celles-ci, centrées sur les pratiques d'ascèse des yamabushi ${ }^{23}$. Les interdictions de Meiji en provoquèrent l'arrêt en I869 (Meiji 2). D'une longueur totale de 175 kilomètres et comportant 50 étapes rituelles, ce trajet part du mont Hōman et se déploie en boucle à travers l'ensemble du massif des Trois cantons. Il en suit la ligne de crêtes sur quelque 80 kilomètres, avant de redescendre par la côte pour retourner au mont Hōman. Il monte ainsi d'abord vers le nord en passant

21. Kaibara Ekiken 貝原益軒 (éd.), Cbikuzen no kuni zoku fudoki 筑前国続風土記, 1977 ( ${ }^{\mathrm{e}}$ éd. 1688-1710), p. 40I.

22. Notamment le Katsuragi bucbū banaku annai 葛城篻中花供案内, Bunka, 9 (1812) rédigé par Ishii-bō 不井坊, dans Mori Hiroko 森弘子, Hōman-zan no kankyō rekishi gaku teki kenḱkȳ p. 542-599; et Anne Bouchy, "'Initiatic landscape' and Shugendō mountain-entry », Proceedings I48 (2012).

23. Minami.ishi Takeshi 南石武 (éd.), Kasuya yōroku 粕屋要録, 1968, p. 401. 
par le mont Sangun et Satani, puis traverse la commune actuelle de Sasaguri en suivant sa ligne de crêtes en demi-cercle depuis le sud par le mont Wakasugi, puis la Crête de l'Ours 熊ヶ尾 au-dessus d'Arata 荒用 en direction de l'est, pour descendre, toujours en suivant la crête, vers la vallée du/des Yamabushi (où avait lieu un arrêtretraite de sept jours) ${ }^{24}$, avant de remonter par Gō-no-haru par la crête Shira.ishi ウ́ 石 (de la Pierre blanche), ligne frontière des trois cantons, vers les monts Unebaru et Hokotate. De là, il descend vers le sanctuaire de Haginoo (où se déroulait un rituel), il traverse la vallée pour gagner au nord-ouest le mont Katsuragi 娄城। (actuel mont Mitsugashira) en passant devant l'actuel Raion-ji 雷音寺 ( $\left.n^{\circ} 49\right)$ pour atteindre le col. Quittant Sasaguri, il passe ensuite par les sommets Shura 首羅獄, Kumano 熊野獄, Inunaki 犬鳴山, Hoko 鉾之峰, Nabiki 㦄獄, Shaka 釈迦獤, Toda 戸田獄 avant d'arriver au mont Kudai-ji 孔大寺山, où aboutit ce parcours de crêtes vers le nord et commence le chemin de retour de 95 kilomètres, vers le sud et le mont Hōman, en traversant villes et villages ${ }^{25}$. On voit donc que le parcours shugen dépasse les limites micro-locales. Bien plus, le long de son trajet perpétué dans la longue durée, l'acte rituel de l'entrée dans la montagne du groupe Hōman n'a cessé d'unir en une communauté régionale transfrontalière, géographiquement éclatée, mais rituellement solidaire, les nombreux et divers groupes sociaux résidant sur le territoire qui, aujourd'hui, correspond aux onze villes et communes actuelles de Dazaifu 太幸府, Umi 宇美, Sue, Sasaguri, Hisayama, Miyawaka 宮若, Koga 古賀， Munakata, Shingū 新宮, Fukuoka, Kasuga 春日.

Ce parcours rituel circulaire, emprunté à intervalles réguliers par les yamabushi, et plus couramment par les chasseurs et tous les usagers du territoire montagnardforestier de cette région, est un exemple, parmi d'autres, des dynamiques de circulation qui traversent les espaces forestiers. Il montre à quel point les montagnes, loin d'être des barrières, sont, par leurs lignes de crêtes où se rejoignent les territoires limitrophes, des espaces de passage et de lien, du moins tant que la marche a été le moyen ordinaire de déplacement. Ce fut le cas jusqu'à notre époque. Tous les adultes actuels des villages les plus éloignés du centre de Sasaguri nous ont dit que, dans leur enfance, ils allaient tous les jours à l'école à pied, depuis Nomiyama au nord à $6 \mathrm{~km}$ (par exemple, Ino.ue Haruo 猪上春雄, né en I9II), comme depuis Wakasugi au sud à $4 \mathrm{~km}$ (ainsi, Takase Kakumyō 高瀬覚明, né en 1952). Le transport de produits alimentaires, de construction, ou autres a continué à se faire à dos d'homme (et de femme) jusque dans les années 1950, partout où les seules voies de circulation étaient des chemins de terre. Franchir les crêtes est plus rapide que de contourner le pied des montagnes. Bien plus, seuls les chemins de lignes de crêtes permettent de ne pas se perdre. Telle est l'une des règles encore en vigueur aujourd'hui dans

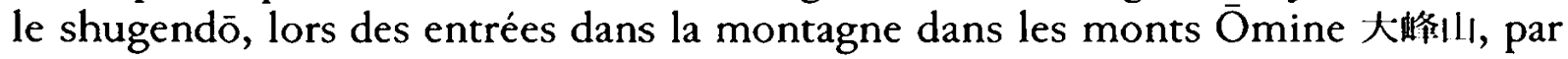
exemple.

24. Voir Mori Hiroko, Hōman-zan no kankyō rekishi gaku teki kenkyū, zoo8 et dans ce même volume, "Le mont Wakasugi, son sanctuaire et le shugen dans l'histoire de Kyūshū et de l'Asie de l'Est ", p. 207-208.

25. Mori Hiroko, op. cit., p. 566. 
En outre, la conception même de la montagne diffusée par le shugendō a contribué à construire une vision autre de l'univers montagneux recouvert de forêts, qui intègre celui-ci dans un vaste maillage signifiant du monde. En effet, le parcours d'entrée dans la montagne du shugen du mont Hōman a été assimilé à une circulation dans les mandala de la Matrice et du Diamant qui sont les montagnes elles-mêmes ${ }^{26}$. Les lieux des cinquante étapes, dont vingt-deux en montagne, ont été identifiés un à un à une entité des mandala. L'ensemble étant donné à voir, à expérimenter et à penser comme étant aussi autre chose que la montagne-forêt physique parcourue ordinairement. Pour les initiés (qui étaient aussi des usagers coutumiers de ce même espace), cette vision grandiose de l'espace double ainsi celle de l'usage quotidien et topographique. Elle vient enrichir la conception coutumière communément partagée par les habitants - dans laquelle elle est ancrée - , conception voulant que la montagne soit le domaine des dieux, des bouddhas et des morts, et donc objet d'interdits, de règles et de ritualités. Ainsi, les pratiques sbugen, qui font emprunter régulièrement ces trajets aux adeptes de la région et leur font rencontrer sur leur passage les populations locales villageoises et citadines hors des limites microlocales, englobent dans des dynamiques socioreligieuses, politiques et économiques à l'échelle de la région tous les petits groupes locaux rattachés au shugen du mont Hōman et les relient eux-mêmes entre eux. Par de tels biais, se sont transmises dans les communautés locales des pratiques et une conception de l'espace familier qui ne réduit pas celui-ci à sa seule délimitation territoriale et administrative. Par là, cet espace a en effet pu être considéré comme étant également le tissu de trajets réunissant entre eux des points, des pôles terrestres, et, par-delà toutes les limites matérielles, ceux-ci à des points idéels (mandala) qui donnent à penser l'univers, visible et invisible, dans sa globalité.

Il est intéressant de noter, tout d'abord, que cette circulation au cour des montagnes du massif des Trois cantons a été entretenue tout au long de l'époque d'Edo, époque au cours de laquelle les communautés villageoises étaient administrativement closes et soumises à de sévères règlementations à la fois internes et externes. Ces dernières étaient imposées par le fief, notamment en ce qui concerne les déplacements des habitants entre l'intérieur et l'extérieur, ce qui contribua certes à construire une forte conscience du dedans et du dehors. Ensuite, il faut mentionner, qu'à cette même époque, la circulation au-delà des limites villageoises et montagneuses n'était pas limitée aux spécialistes du religieux (qui étaient aussi des habitants de ces villages) ou à ceux des activités forestières (charbonniers, bûcherons, collecteurs de végétaux sauvages, chasseurs, travailleurs du bois, itinérants de toutes sortes). Il semble qu'elle ait été également une composante des pratiques matrimoniales locales. Ainsi, à Wakasugi, les épouses venaient préférentiellement de « l'autre côté de la montagne », c'est-à-dire de Satani. Et vice versa. C'est ce qui s'est passé pour un certain nombre des maîtresses de maison actuelles. Il en a été de même pour certains des époux venus en « gendres adoptés ». Les liens de parenté ainsi tissés au cours des âges lient les deux communautés villageoises par-delà la montagne. De

26. Voir Mori Hiroko, Hōman-zan no kankyō rekishi gaku teki kenkyū, 2008 et Anne Bouchy, "'Initiatic landscape' and Shugendō mountain-entry », 2012. 
fait, les gens de Wakasugi (Sasaguri) disent se sentir plus proches des habitants de Satani (Sue) auxquels ils sont " liés par un même sang ${ }^{27}$, que de ceux de Kido ou de Haginoo (Sasaguri), car il y avait « échange de femmes » entre les deux villages reconnaissent certains ${ }^{28}$. Ce fait n'est pas limité à Wakasugi. Nombre de familles de divers quartiers de Sasaguri nous ont dit que les épouses venaient traditionnellement d'autres villages, communes ou villes limitrophes, avec lesquels étaient ainsi établis des liens de parenté préférentiels. "Elles venaient de l'extérieur", nous dit-on un peu partout, «aussi ne connaissaient-elles pas la situation (jijō 事情) de Sasaguri », c'est-à-dire les coutumes et les réseaux de la société locale. Il en était de même pour les gendres adoptés. Tous ces «venus d'ailleurs » devaient donc apprendre les usages locaux pour s'y conformer, mais, à chaque génération, ils apportaient aussi leur part d'innovation ${ }^{29}$. Aujourd'hui, la mobilité individuelle a brisé l'aspect récurrent de ce schéma matrimonial, qui néanmoins subsiste comme une mémoire dans certaines familles.

Les aspects évoqués précédemment permettent de dire, à ce stade de nos investigations, que la circulation est une constante de Sasaguri ; que, par-delà les expressions diverses d'un fort sentiment d'appartenance à la communauté locale restreinte (coïncidant le plus souvent avec les anciens villages), les faits montrent que le «dedans » et le «dehors » doivent y être pris comme des notions relatives, car ils ont fait l'objet de nombreux remaniements successifs en moins d'un siècle d'histoire moderne et contemporaine (ceci a pour corollaire la question : à quelle communauté le sentiment d'appartenance individuel et collectif fait-il référence ?) ; et que l'apparente fermeture topographique du territoire n'a pas conduit la communauté locale à être une enclave socioculturelle. Les montagnes-forêts, principale composante de ce territoire communal, sont ainsi envisagées ici comme des espaces de la vie locale construits par le cheminement de ceux qui les traversent et y circulent pour leur usage, et en font un milieu ouvert vers d'autres espaces qui leur sont contigus et avec lesquels, par le biais de ces trajectoires, ils sont reliés et en interaction. Comme tout espace social, les montagnes-forêts sont des "tissus maillés », ou des "maillages ", terme par lequel je traduis la notion "meshwork » de Tim Ingold, qui lui-même rend ainsi le concept « texture », proposé par Henri Lefebvre dans sa réflexion sur la production de l'espace ${ }^{30}$. Loin d'être des lignes reliant seulement un point à un autre, les trajectoires sont conçues comme des possibilités d'interactions multiples, par lesquelles sont générés à la fois ceux qui en sont les acteurs en tant qu'êtres sensibles et sociaux, et l'espace où ces trajectoires se

27. «Chisuji ga tsunagatteiru»血筋がつながつている.

28. Cf. Suzuki Masataka, "Continuités et transformations de la société locale " dans ce volume, p. 4I, note 43 : « les Gōya 合屋 sont nombreux à Utani et à Satani [...]. Les Yasugōchi adoptent des gendres de Satani. On dit que là où le réseau des eaux est commun, comme à Wakasugi et à Kasuya, on pratiquait autrefois les échanges matrimoniaux pour devenir parents. »

29. Cf. Ishikawa Toshiko, «Les rites annuels de la maison et des communautés locales en transformation. Intérieur et extérieur des lieux de vie communautaires », dans ce volume, p. 486-497.

30. Tim Ingold, Lines. A brief bistory, 2007 et Tim Ingold, Being alive: Essays on Movement, Knowledge and Description, 20II, p. 84. Henri Lefebvre, La production de l'espace, 2000 ( $\mathrm{r}^{\mathrm{e}}$ éd. 1974), p. 132-195. 
déploient comme autant de lignes de vie. Il s'agit donc de comprendre les propriétés et les modalités d'interférence de ces maillages dans le processus d'élaboration continue des nombreux espaces sociaux, et la façon dont sont ainsi définis des intérieurs et des extérieurs fluctuants selon les positions.

La montagne-forêt : réalités et concepts - les recherches japonaises

- Quelques chiffres

Les 2395 hectares des forêts actuelles de Sasaguri représentent aujourd'hui (2010) $61,56 \%$ du territoire communal ( 3890 ha). Jusqu'à une date récente ce chiffre était de 67 ou $70 \%{ }^{31}$, ce qui correspond aux $67 \%$ du pourcentage national moyen d'occupation des sols par les forêts $\left(25100000 \mathrm{ha}^{32}\right.$ de forêts pour un territoire d'une superficie totale de $37800000 \mathrm{ha})^{33}$ et dépasse de $16 \%$ l'étendue forestière moyenne dans le département de Fukuoka (45\%:222369ha de forêts sur $497617 \mathrm{ha})^{34}$.

Il est important de souligner que, physiquement, montagnes et forêts sont quasiment coextensives dans la commune comme à l'échelle du pays. Dans la langue, ceci se traduit par une proximité conceptuelle et terminologique des deux espaces. S'il existe bien des termes distincts pour désigner la montagne — yama 山 —, et la forêt, 一 mori 森 ou sbinrin 森林, ou les bois, bayashi 林 —, l'appellation usuelle sanrin 山林, c'est-à-dire " montagne-forêt ", " forêt de montagne ", est préférentiellement employée pour parler des forêts. L'accent y est plutôt mis sur la montagne, et c'est très généralement «yama » W, que l'on utilise dans le langage courant pour parler de ces territoires, conçus comme une seule et même entité. L'expression « aller, être dans la montagne » est employée pour dire aussi que l'on va ou que l'on est en forêt. Inversement, lorsqu'on parle de la forêt, c'est aussi de la montagne dont il est question. On note également que la « montagne » est une notion qui recouvre l'ensemble des espaces qui sont hors des plaines agricoles et urbaines. Aussi cette notion n'est-elle pas exclusivement liée aux hauts sommets, mais englobe aussi les collines de faible élévation. Le mont Wakasugi $(655 \mathrm{~m})$ et les collines de Kana.ide, Takata ou Oto.inu (une centaine de mètres) sont semblablement appelés « montagnes ", ainsi que, par exemple, le mont Hiko 英彦山 ( $1200 \mathrm{~m}$, Soeda 添田町, département de Fukuoka). On verra néanmoins que, dans les pratiques locales, il existe toute une gamme de termes, notions, règlementations visant à distinguer très finement ces espaces relativement aux systèmes de propriété, de gestion et d'usage. Ces notions relatives aux espaces physiques sont essentielles, car elles sous-tendent

3I. Sasaguri-chō bunkazai senmon-iinkai 篠栗町文化財専門委員会 (éd.), Sasaguri chōshi. Minzoku-ben 篠栗町誌·民俗編, 1990, p. 369.

32. 25096987,45 ha en 20 Ir (d'après les relevés disponibles sur le site internet officiel de l'Office de la sylviculture, Rin.yachō 林野庁 [Forestry Agency], <http://www.rinya.maff.go.jp> [date du dernier accès II/08/2012]).

33. $377944 \mathrm{~km}^{2}$.

34. Superficie forestière départementalc : $29^{\circ}$ rang national. Tous les chiffres conccrnant le département de Fukuoka viennent du site internet du département de Fukuoka (<http://www. pref.fukuoka.lg.jp>), 20II [date du dernier accès 05/08/2012] et du site Rin.yachō, 2011 (voir ci-dessus note 32 ). 
et reflètent une vision du monde et des qualifications de l'espace qui, à leur tour, légitiment des formes de propriétés, des systèmes d'usage, des rapports de pouvoirs et des conflits. Ces éléments conceptuels et pratiques se situent à l'articulation de multiples dimensions socioéconomiques et de leurs expressions culturelles (religieuses, symboliques, littéraires, légendaires) et, plus largement, de l'imaginaire.

L'époque récente voit la diminution drastique des activités liées à la montagne et à la terre en général. Si, entre 1990 et 2000 le nombre d'agriculteurs de Sasaguri est passé de 496 à 339 (soit de 2,13\% à I,I5\% de la population, qui, dans le même temps, s'élève de 23267 à 29389 habitants) ${ }^{35}$, en 1963, les II 48 agriculteurs représentaient $21,5 \%$ des 5342 habitants, et $22,8 \%$ si on ajoute ceux (69) qui faisaient des travaux spécialisés dans les forêts ${ }^{36}$. Mais les proportions sont encore bien différentes pour les époques antérieures. Au début de Meiji (des années I870 à la fin du XIX ${ }^{c}$ siècle), la majorité des 3802 habitants des villages formant l'actuel Sasaguri, possédaient et exploitaient 495,85 ha de terres et 1378,46 ha de forêts villageoises ${ }^{37}$, le reste des forêts étant devenu majoritairement propriété de l'État à Meiji. Une enquête faite en 1907 et en 1908 (Meiji 40 et 4I) par les responsables des villages, pour un recensement national, nous donne des précisions sur Sasaguri (Sasaguri, Kana.ide, Takata, Haginoo) et Seto (Onaka, Wakasugi, Oto.inu, Wada, Tsubakuro) qui à l'époque ne formaient pas encore une entité communale unique ${ }^{38}$.

$50 \%$ des 2692 habitants du village de Sasaguri vivaient de l'exploitation des terres et de l'agriculture, et l'exploitation des forêts représentait alors $50 \%$ du chiffre total des productions locales; tandis que $63 \%$ des 2459 habitants du village de Seto vivaient principalement ( $25 \%$ exclusivement) de l'agriculture (rizières et champs secs), les produits agricoles représentant la majorité de la production locale, devant le produit des mines de charbon, et les forêts y étaient principalement exploitées par des coopératives de villages ou de particuliers (nous y reviendrons).

Il faut noter d'abord que, dans ce contexte, ce sont majoritairement les villageois, pratiquant aussi l'agriculture, qui s'occupent des espaces forestiers. Il y a donc cumul des activités agricoles et sylvicoles.

Ensuite, ces chiffres reflètent bien les caractères des bouleversements qui ont touché les activités agricoles et sylvicoles dans l'ensemble du pays depuis Meiji. Si la population du pays a augmenté régulièrement depuis 1868 , avec une légère inflexion

35. Chiffres de l'état des lieux fait par la mairie en 2006 (Sasaguri-chō kikaku kōhōka 篠栗 町企画広報課 (éd.), Sasaguri-chō. Chösei yōran, shiryöben 篠栗町:町势要覧·資料編, 2006, p. 3). Dans le même temps, le nombre de foyers vivant de l'agriculture passe de 392 (I815 personnes) à 349 (990 personnes), et les espaces agricoles diminuent de 313 ha (rizières $26 \mathrm{I}$ ha, champs $52 \mathrm{ha}$ ) à 265 ha (rizières 227 ha, champs $38 \mathrm{ha}$ ) soit environ $6 \%$ de l'occupation des sols.

36. Chiffres de l'état des lieux fait par la mairie en 1964 (Sasaguri-chō 策柴町 (éd.), Sasaguri. Chōsei yōran ささぐり:町勢要覧, 1964, p. 9).

37. Nishi Nihon bunka kyôkai 西日本文化協会 (éd.), Fukuoka-ken chiri zenshi 福岡県地理 全誌, vol. 6 de la section documents historiques (cités également dans Sasaguri chōshi, p. 218).

38. Archives du Centre de documents historiques et matériaux ethnographiques (Sasaguri rekishi minzoku shiryō shitsu 策栗歴史民俗資料室, abrégé ci-après en Sasaguri shiryō shitsu) : Tō Kihachirō 藤喜八郎 (éd.), Kasuyagun Sasaguri-son sonze, kan 糟屋郡策栗村々是·完, 1907, et Takahashi Katsubee 高橋勝兵衛 (éd.), Kasuyagun Seto-son sonze, kan 糟尾郡勢門村々是·完, 1908. 
due à la guerre en 1945 (34806000 d'habitants en 1872, 50577000 en 1912, 72147000 en 1945,80002000 en 1948 , 100196000 en 1967 et 128056000 en $2010^{39}$, soit une multiplication par trois en un siècle) et une baisse ces deux dernières années (127610000 en 2012), dans le même temps les courbes des activités par secteurs professionnels se sont quasiment inversées. Le secteur secondaire, qui représentait Io\% de la totalité des emplois au début $\mathrm{du} \mathrm{xx}^{\mathrm{e}}$ siècle, a été en légère augmentation jusqu'aux années I980-1990 (36,6\%), à partir desquelles il se réduit progressivement ( $24,8 \%$ en 2010 ). Cet affaiblissement est dû à l'explosion du secteur tertiaire, des services, qui, dès la fin du $\mathrm{XIX}^{e}$ et au début du $\mathrm{XX}^{e}$ dépassait déjà le secteur secondaire avec $20 \%$ des emplois, mais est passé à $40 \%$ dans les années 1960 pour atteindre aujourd'hui plus de $70 \%$. Durant cette même période, le secteur primaire (agriculture, sylviculture, pêche, aquaculture) qui occupait plus de $80 \%$ de la population active début Meiji, descend à $70 \%$ au début du $\mathrm{xx}^{e}$ siècle, connaît une remontée ( $55 \%$ ) entre l'après-guerre et 1950 , pour ensuite ne cesser de diminuer. Il atteint le niveau du secteur tertiaire à la fin des années 1950 (40\%), puis celui du secteur secondaire au milieu des années 1960 (25\%), pour ne plus représenter aujourd'hui que $4 \%$ des activités. Dans le département de Fukuoka, en 20I0, les actifs du secteur agricole constituent 3,21 \% de la population du département, ceux du secteur sylvicole pas même I\%.

\section{- Les travaux sur les montagnes-forêts en etbnologie du Japon}

Pourtant, étant donné l'importance de l'étendue des montagnes-forêts sur le territoire national, tant que l'économie de subsistance est restée ancrée dans le secteur primaire, la majorité de la population vivant de l'agriculture (polyculture et riziculture irriguée) s'est occupée également, pour une part plus ou moins grande et selon des techniques diverses, de la gestion et de l'exploitation de ces espaces montagneux au milieu ou à proximité desquels sont situés les villages. Et c'est sur ces communautés villageoises que s'est focalisée l'ethnologie du Japon jusque dans les années 1970-1980. Née au début du $\mathrm{XX}^{\mathrm{e}}$ siècle, cette discipline a en partie pour origine un questionnement sur les difficultés rencontrées à cette époque par les habitants des villages, notamment des zones montagneuses et reculées ${ }^{40}$. Sur cet arrière-plan, la question des montagnes-forêts et des arbres a bien sûr été l'un des axes de recherche importants de l'ethnologie du Japon. Elle a d'abord été traitée dans le cadre d'études portant sur les activités de subsistance et de production, et abordant principalement les montagnes-forêts et les arbres sous leur aspect de ressources matérielles, culturelles et symboliques. Les recherches se sont intéressées ainsi à eux en tant qu'objets de techniques traditionnelles et de pratiques sociales coutumières formant systèmes, d'une part $^{41}$ et, de l'autre, en tant que lieux privilégiés et éléments du religieux et

39. Tous les chiffres de ce paragraphe proviennent des recensements nationaux (kokusei chōsa 国勢調査 [2007]) disponibles en version papier et sur internet sur le site de l'Agence des statistiques du ministère de l'Intérieur [Sōmu-shō tōkei-kyoku 総務省統計局].

40. Sur l'historique, les domaines et les concepts de l'ethnologie du Japon, voir Anne Bouchy, "De l'ethnologie du Japon : par qui, où, comment ? ", Ateliers 30 (2006), p. 63-99.

41. Le cadre de cette contribution ne permettant pas une présentation bibliographique étendue, seuls les principaux axes d'approche et quelques travaux majeurs sont ici mentionnés. 
du symbolique. Les premières ont fourni d'innombrables études sur l'agriculture irriguée, sèche et sur brûlis, la pêche, les activités cynégétiques, les systèmes des communaux et les utilisations coutumières de ces espaces pour le combustible, le fourrage et les engrais ; ainsi que sur la sylviculture et les usages de la forêt en relation avec les différents modes d'exploitation des milieux. Les secondes se sont centrées sur les rites, les pratiques cultuelles et les systèmes religieux liés à la montagne. Elles ont ainsi permis de mettre en lumière l'éventail impressionnant des pratiques funéraires, le panthéon et les croyances, les représentations et leurs diverses expressions, notamment la littérature orale ; le dieu de la montagne, au cœur des constructions rituelles et symboliques, faisant pendant ici à l'omniprésence de la montagne-territoire. C'est aussi ce qui a conduit, ensuite, à « (re)découvrir » le shugend $\bar{o}^{42}$. Les travaux les plus anciens ont eu tendance à démanteler, dans l'observation et l'analyse, les pratiques, les techniques, les objets en éléments distincts, et séparés de leurs milieux, alors que les recherches actuelles abordent ces questions à partir de problématiques d'ensemble. À partir de la fin des années 1960 et du début des années 1970, grâce aux travaux innovants sur la « culture de la zone asiatique de la laurisylve (shoyojojurin 照葉樹林) $)^{43}$, sur les populations des montagnes qui pratiquent les cultures sur brûlis et donnent rituellement la prééminence aux tubercules et aux produits de la chasse, est remise en question la centralité de la riziculture ${ }^{44}$. $\mathrm{Par}$ là, sont aussi réévalués les liens complexes entre l'univers des montagnes-forêts et celui des riziculteurs. Avec ces nouvelles orientations et parallèlement à l'essor de l'ethnologie urbaine, à partir des années 1970-1980 se dégagent d'autres lignes de recherches, appréhendant la montagne-forêt en tant qu'élément constitutif majeur de la conception de l'espace (social, rituel, cultuel, symbolique). Puis, au début des années 1990, les questions de l'environnement et de la nature émergent au premier plan et contribuent encore à orienter l'intérêt vers les milieux eux-mêmes, et donc les montagnes-forêts, considérés non plus seulement comme objets (des actions humaines), mais comme milieux vivants à partir desquels la société et ses pratiques coutumières sont observées.

En ce domaine, la transformation des approches en ethnologie du Japon a suivi une courbe qui s'est infléchie au cours du $\mathrm{Xx}^{e}$ siècle de façon aussi importante que celle des trois grands secteurs d'activités économiques. Mais l'intérêt pour l'environnement s'est développé à l'inverse de la courbe descendante du secteur primaire, et parallèlement à l'émergence des problèmes environnementaux. Le changement vaut pour les objets et les méthodes, mais concerne aussi l'attitude des chercheurs. Au début du siècle dernier, la plupart d'entre eux, même s'ils habitaient en ville, avaient une connaissance et une expérience directes des milieux montagneux et forestiers, alors qu'aujourd'hui ils sont en majorité natifs et habitants de mégalopoles. Trois lignes majeures de travaux sont

42. Voir Anne Bouchy, « La cascade et l'écritoire. Dynamique de l'histoire du fait religieux et de l'ethnologie du Japon : le cas du shugendō », BEFEO 87 (2000).

43. Littéralement «forêt à feuilles luisantes ", c'est-à-dire sempervirente ; désigne la laurisylve.

44. Notamment les pionniers : Sasaki Kōmei 任々 木高明, Inasaku izen 稻作以前, 1971 ; Sasaki Kōmei, Shōyōjurin bunka no michi 照集樹林文化の道, 1982. Tsuboi Hirofumi 坪井洋文, Imo to Nibonjin イモと日本人, 1979 ; Tsuboi Hirofumi, Ine wo eranda Nibonjin 桴を選んだ月本人, 1982. 
actuellement en plein essor. Elles se situent au confluent de l'ethnologie du Japon, de l'anthropologie et de l'écologie, et intègrent des problématiques des sciences naturelles,

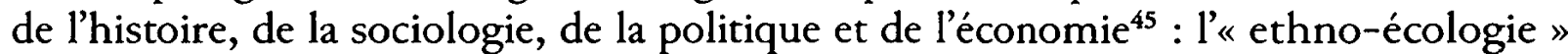
(seitai minzokugaku生態民俗学) ou étude, dans la longue durée, des savoirs et usages coutumiers en relation avec les différents milieux de vie ${ }^{46}$, l'ethnoscience (minzoku shizenshi 民俗自然誌) ou « ethnographie des savoirs et usages coutumiers de la nature 》, qui combine ethnographie et approches naturaliste et économique des pratiques et connaissances coutumières ${ }^{47}$, et l'ethnologie/sociologie de l'environnement (kankyō minzokugaku/shakaigaku環境民俗学・社会学) qui appréhende les rapports à l'environnement par le biais des systèmes sociaux coutumiers ${ }^{48}$. Dans ce contexte, les travaux sur les milieux forestiers ont contribué à renouveler toutes les approches antérieures, en fournissant à la fois une ethnographie détaillée et une réflexion qui ouvre des pistes nouvelles, notamment sur toutes les pratiques communautaires, l'état actuel et les possibilités futures de la gescion et de l'usage des forêts. Ces thématiques sont aujourd'hui abordées en tenant compte de l'apport conjugué des travaux japonais et occidentaux sur la question. Tous ces débats actuels sont la trame de fond de mon interrogation sur les rapports communautaires aux milieux forestiers de Sasaguri. Partant de l'examen des relations pratiques et coutumières aux milieux de vie, et pour mieux faire apparaitre la complexité du rapport socio-environnemental réel aux forêts, ma démarche a été de tenter de faire s'entrecroiser les approches et les problématiques relatives à l'« environnement » et celles portant sur le symbolique et le fait religieux. Certaines de ces problématiques appartiennent à des champs d'études distincts, qui ne se rencontrent pas nécessairement autour de cet objet commun. Par-delà les difficultés et les risques inhérents à ce rapprochement, ce qui a motivé ma tentative est la richesse de ces convergences, dont les limites de cette contribution permettent seulement de donner un aperçu.

- La question $d u$ sato-yama 里山

Jusqu'au $\mathrm{XX}^{e}$ siècle, les forêts en tant que milieu ont surtout été étudiées par les géographes, les agronomes, les économistes, ainsi que par les historiens et les légistes pour les communaux. Mais à partir de la fin des années 90 , une thématique a réuni non seulement tous ces chercheurs, les ethnologues et les sociologues, mais aussi les acteurs de la vie publique, politique et économique, et, plus largement, les citoyens, le grand public. Il s'agit de la question de la montagne-forêt villageoise, le

45. Pour une synthèse de ces trois lignes d'approche, voir Suga Yutaka 营豊, "Shizen : shizen wo meguru minzoku kenkyū no mittsu no chōryū » 自然:自然をめぐる民俗研究の三つの 潮流, Nibon minzokugaku 日本民俗学, 227 (2001), p. 14-29. Nomoto Kan.ichi 野本筧一, Fukuta Ajio 福田アジオ et al., éd. Kankyō no minzoku 環境の民俗, Kōza Nihon minzokugaku 講座:日本 の民俗学 4, 1996, vol. 4 (première compilation sur les questions de l'environnement dans les collections d'ethnologie du Japon).

46. Nomoto Kan.ichi 野本筧一, Seitai minzokugaku josetsu 生悲民份学序説, 1987.

47. Shinohara Tōru 筗原微, Shizen to minzoku 自然と氏俗, 1990.

48. Torigoe Hiroyuki 息越船之, éd. Kokoromi toshite no kankyō minzokugaku 試みとしての㻴 境民俗学, 1994. 
sato-yama 里山 ( montagne du village $»^{49}$ ). Le terme et le concept sont devenus des référents incontournables dans les discours et les pratiques actuelles concernant les milieux forestiers. En outre, c'est une notion-clef pour comprendre les dynamiques qui n'ont cessé de construire les montagnes-forêts de Sasaguri. Aussi je présenterai ici brièvement cette question avant d'entrer dans le monde forestier de Sasaguri.

Désignant l'espace de montagnes et de collines proches des villages où se trouvent les bois, les rizières et les champs, les landes à usages coutumiers, c'est-à-dire les écosystèmes placés directement sous l'influence des actions anthropiques, le sato-yama est un terme ancien qui a été "(ré-)inventé » au milieu du $\mathrm{xx}^{\mathrm{e}}$ siècle ${ }^{50}$. L'invention en question serait en fait plutôt celle de l'acception actuelle du terme dans la conjoncture particulière de notre époque. Car, selon les lieux, cet espace, qui a en effet pu être désigné anciennement par divers noms, tient un rôle essentiel à l'échelle du pays. Partout on retrouve l'existence d'une classification des catégories spatiales qui en tient compte ${ }^{51}$. Cette qualification spatiale est établie en fonction de trois paramètres : les types de ressources (végétales, animales, aquatiques, minérales, etc.) et donc les types d'usages liés aux différents milieux montagneux, ensuite le type et l'élévation du terrain, et enfin la distance par rapport aux habitants-usagers. Il faut noter que cette distance, ici en l'occurrence la proximité du sato-yama, n'est pas seulement physique, mais qu'il s'agit surtout d'une proximité de relations. C'est la fréquence de l'usage de cet espace (qui peut être plus ou moins éloigné physiquement des localités, mais n'en est jamais très loin) qui caractérise et définit le satoyama. C'est, de fait, un espace à usage quotidien, coutumier, auquel les habitants ont recours dans la vie de tous les jours. Ceci a permis de définir aussi le sato-yama comme «bois à usage agricole » (nôyō-rin 農用林). Mais c'est là une acception limitée du terme ainsi centré sur la consommation de la forêt, et qui ne comprend pas les dimensions géographiques, paysagères, multi-relationnelles entre les habitants et cette forêt. Comme c'est le cas à Sasaguri, cet usage et ce mode relationnel avec

49. On note ici l'usage du terme "montagne » (yama), et non de celui de "forêt ", pour désigner une entité principalement définie par sa couverture végétale (forêts, bois, champs, etc.).

50. Bien que l'on retrouve ce terme utilisé dans les textes anciens, l'« invention » en est revendiquée par le spécialiste d'agronomie Shidei Tsunahide (Shidei Tsunahide 四手井網英, Shinrin 森林, coll. “Mono to ningen no bunkashi »ものと人問の文化进, 53, vol. 1, 1985; Shidei, Sbinrin II 森林 2, coll. “Mono to ningen no bunkashi »; 53, vol. 2, 1998 ; Shidei, Shinrin III 森林 3, coll. "Mono to ningen no bunkashi " 53, vol. 3, 2000 ; Shidei, Shinrin wa mori ya hayashi dewanai. Watashi no shinrinron 森林はモリやハヤシではない——私の森林論, 2006), ce qui a été remis en cause par d'autres chercheurs.

51. Par exemple à Yoshino (département de Nara), sato-yama, la " montagne du village », ce sont les champs secs et les bois de feuillus sur les pentes autour des habitations. Cet espace ainsi clairement distingué de trois autres catégories spatiales : uchi-yama 內山, la « montagne de chez nous/intérieure ", c'est-à-dire les terres pour brûlis, les champs de muriers, les forêts à charbon de bois ; oku-yama 奥l山, le « fond de la montagne/la montagne profonde ", c'est-à-dire la forêt sur les pentes des montagnes dont les arbres sont utilisés pour faire, notamment, le bois de charpente, et où est pratiquée la chasse ; et take 获, la "montagne élevée/les sommets ", c'est-à-dire les plus grandes hauteurs où pousse la forêt auto-régénérée. Sasaki Kōmei 传々本高明, Nibon bunka no tayösei H本文化の多㥞性:, 2009, p. 126-127. 
le milieu forestier proche, répétés au cours des siècles, ont constamment modifié l'écosystème dont dépendent les groupes humains eux-mêmes.

L'émergence sur le devant de la scène de la question du sato-yama tient à plusieurs facteurs, dont les problèmes environnementaux que suscitent les crises dans la gestion des espaces montagneux à partir de la seconde moitié du $\mathrm{Xx}^{\mathrm{e}}$ siècle. En effet, à partir des années 1960, pour des questions économiques locales et globales (révolution du combustible, montée des coûts du bois, etc.), ont succédé à la surexploitation et à la déforestation massive soit l'abandon et l'« ensauvagement » des montagnes-forêts, soit leur destruction pour urbanisation intensive, avec tous leurs effets néfastes subséquents sur l'environnement villageois ${ }^{52}$. Les prises de parole publiques sur ces problèmes se sont faites de plus en plus contestataires dans les années 1990, notamment avec la montée des mouvements de défense de l'environnement ${ }^{53}$. L'urgence d'une revalorisation de la petite montagne (dite nature "secondaire ») pour sauvegarder les biotopes et les écosystèmes s'est manifestée au moment même où cet espace s'est trouvé menacé de destruction complète par l'urbanisation, la pollution ou l'abandon aux friches. Face à la dégradation des milieux naturels proches, des actions ont ainsi été menées pour attirer l'attention sur la nécessité de préserver non pas des lieux ou des espèces isolés, mais des ensembles naturels, et de revaloriser ces espaces « communs », " ordinaires » délaissés au profit des hautes montagnes ou autres lieux remarquables (qualifiés de nature "primaire », spontanée). Car, dans le même temps, ces derniers devenaient, eux, des objets de patrimonialisation pour leur rareté ou leur caractère d'exception.

Ainsi le sato-yama émerge-t-il comme notion partagée, mais transformée, hors du cadre de ses usages traditionnels au moment même de sa mise en danger, voire de sa disparition, et ce, non seulement à l'intérieur du Japon mais aussi à l'extérieur. Dans ce contexte, ont joué un rôle majeur l'exposition internationale de Nagoya en 2005 organisée autour du thème "la sagesse de la nature " ${ }^{54}$ sur les lieux même d'une forêt villageoise ${ }^{55}$, et la dixième conférence internationale sur la biodiversité

52. Voir notamment Arioka Toshiyuki 有岡利幸, Sato-yama II 里ll 2, coll. « Mono to ningen no bunkashi ", 118 , vol. 2, 2004, p. 67-98.

53. Ces mouvements ne sont pas nés à cette époque. Ils perpétuent et renouvellent notamment ceux qui avaient été engagés dès les années 1950, lors des combats contre les grandes pollutions du $\mathrm{Xx}^{\mathrm{e}}$ siècle, dont celle de Minamata 水俣 (1932-68) contre les effets de laquelle la lutte et les procédures judiciaires sont toujours en cours en 2012.

54. «Shizen no eichi 》自然の知 (25.3-25. 9. 2005). The 2005 World Exposition, Aichi, Japan est la traduction anglaise du nom officiel : « 2005 nen Nihon kokusai hakuran-kai 件日本国際博 覧会》 (《Exposition universelle du Japon, 2005»).

55. Sur les débats et enjeux intellectuels, le contenu et les divers arrière-plans de l'exposition internationale de Nagoya 2005, voir la série d'articles de Sophie Houdart, "L'image ou sa dissolution au moment de la préparation de l'Exposition internationale japonaise de 2005 ", Autrepart 24 (2002) ; Houdart, « Paysages et jardins : un autre regard » Ebisu 28 (2002); Houdart, "Exposer la nature d'hier, construire la nature de demain. L'Exposition internationale japonaise de 2005 ", $1^{\text {er }}$ Congrès du Réseau Asie, 2003 ; Houdart, "Le sato-yama comme arène politique au moment de la préparation de l'Exposition internationale japonaise, Aichi 2005 ", Japon pluriel, 2004 ; Houdart, "Un chaos savamment ordonné..." : l'élaboration conceptuelle de l'Exposition internationale japonaise de 2005 ", Ateliers 30 (2006). 
de $2010(\mathrm{COP} \text { IO })^{56}$, qui s'est déroulée elle aussi à Nagoya et à laquelle a été adjointe «L'initiative satoyama ${ }^{57}$. L'action des divers médias fut, elle aussi, déterminante pour la diffusion de l'image et du terme de sato-yama ${ }^{58}$. Alors que le sato-yama reste d'abord une question locale, ces manifestations l'ont fait accéder à une dimension internationale. Ceci a également eu un impact sur les travaux d'ethnologie du Japon qui aujourd'hui ont intégré cette notion dans leurs thématiques de recherche.

Mise au cœur de polémiques dans lesquelles s'opposent les points de vue « anthropocentrés » (le sato-yama comme modèle du développement durable) et « naturocentrés » (contre le sato-yama, nature anthropisée, obstacle à la régénération naturelle), la montagne villageoise est devenue un champ privilégié pour débattre de questions de fond comme celle de la conception de la "nature », ou celle de la gestion politique et économique de l'environnement. Or les positions, les choix et les décisions adoptés sont souvent ambigus. Car ils sont pris en tenailles entre les argumentaires de la conservation, de la préservation, de la protection et ceux de la mutation en espaces récréatifs à l'usage des citadins. Proposé comme un modèle idéal et universel de relations (un mode de vie « ancestral ») entre les groupes humains et leur milieu (la "nature ») lors de COP ıo, le sato-yama est le lieu d'où la société japonaise peut certes établir un dialogue et des liens avec les autres pays de la planète. Mais donné pour essentiel à l'identité japonaise en tant que " paysage » matriciel, il peut aussi être instrumentalisé par le nationalisme culturel. Pris entre l'idéel, l'idéal, et les réalités des enjeux et des constructions locales ou planétaires, la « montagne du village » est donc plus que jamais au cœur de dynamiques traversant de multiples « dedans » et « dehors », qui l'englobent, la construisent ou la détruisent. C'est aussi dans ce contexte que prend tout son sens une investigation de réalités locales précises, afin de voir comment est aujourd'hui envisagée et gérée concrètement cette question au confluent de l'héritage, des exigences du présent et des nécessaires transformations pour l'avenir.

56. "COP IO " : $10^{\circ}$ Conférence des parties à la Convention sur la diversité biologique de l'ONU (Kokuren seibutsu tayō-sei jōyaku dai jukkai teiyaku-koku kaigi 国連生物多様性条約 第 10 回締約因会議) 18-29 octobre 2010, qui a adopté le Protocole de Nagoya sur l'accès aux ressources génétiques et le partage juste et équitable des avantages découlant de leur utilisation relatif à la convention sur la diversité biologique.

57. «COP 1o Side Event: Satoyama Governors' Summit»(CBD/COPıо サイドイベント里山 知事サミット) centrée sur la thématique : «The Satoyama Initiative: Toward a Rural Society in Harmony with Nature " (Satoyama, shizen to tomoni ikiru chie, inochi wo hagukumu basho 里山一自然と共に生きる知恵·命を育む場所), 19-25, octobre 2010.

58. Outre la télévision, le cinéma et la photographie, il faut noter l'influence internationale de films d'animation comme «Mon voisin Totoro " (Tonari no Totoro となりのトトロ), réalisé en 1988 par Miyazaki Hayao 宮崎駿 ( ${ }^{\text {re }}$ présentation en France en 1992). 
Forêts, bois et hommes : aujourd'hui, l'héritage historique de Sasaguri

\section{L'bétérogénéité des forêts de Sasaguri}

Physiquement, de quoi sont constitués ces espaces forestiers ? Aujourd'hui, les 2395 ha boisés du territoire communal sont en majeure partie $(2359$ ha, soit $98,49 \%)$ intégrés au parc naturel départemental de Daizaifu ${ }^{59}$, qui s'étend de Haginoo et Yamate 山手 au nord, à Kido, Sannō 山王, Naijū et Wakasugi au sud (carte 3). Ils représentent les plus proches espaces verts de récréation pour les citadins de Fukuoka. Mais cette intégration dans un même ensemble administratif est loin d'être synonyme d'unité végétale. C'est plutôt la pluralité des peuplements forestiers qui en est la caractéristique. La classification des forêts faite par l'Office des forêts (Rin.yachō 林野伃) est organisée selon plusieurs critères : la formation, les types de peuplements, les types de propriété et de gestion, la fonctionnalité. La commune de Sasaguri dispose de classifications et de cartes forestières établies à partir de la propriété, de la gestion et de l'usage, mais les indications sur la formation et la nature des peuplements boisés sont absentes. Il existe une carte à l'échelle du département, sur laquelle seuls quatre types de végétation sont indiqués pour Sasaguri : les rizières (partie orientale), les plantations de conifères (la presque totalité de la surface forestière), à l'intérieur desquelles sont situés deux petits secteurs de chênes à feuilles persistantes (Kido, Naijū), spécifiques de la zone tempérée chaude du Japon oriental et méridional. Mais avec ces seules données officielles, il est impossible de se faire une idée exacte de la végétation au niveau micro-local.

Seule l'enquête de terrain fournit des données qui permettent d'élaborer différentes classifications beaucoup plus détaillées des espaces forestiers actuels de Sasaguri. Tout d'abord, à partir des données recueillies sur place, on peut établir un tableau de l'état physique des zones boisées, en retenant conjointement comme critères : les types de formation, la situation géographique et les peuplements. On peut voir ainsi se dessiner quatre grandes catégories : les forêts auto-régénérées, les forêts plantées, les repousses sauvages et les espaces déforestés.

\section{- Les deux sortes de forêts auto-régénérées}

La plus ancienne zone boisée de la commune se trouve à Kana.ide, sur la colline en bordure des habitations et dans l'enceinte du sanctuaire Hōman-gũ 宝満宮 (fig. 2). Simplement appelée par les habitants « la montagne du dessus 》(Ue no yama 上の山), elle surplombe le sanctuaire Hōman-gū et le temple Shūrin-ji 珠林寺 qui la possèdent à part égale. Cette petite forêt dite spontanée (tennen seirin 天然生林60) et auto-régénérée, est pour l'essentiel constituée d'arbres sempervirents de la laurisylve, dont les

59. Dazaifu kenritsu shizen kōen 太莘府県立自然公園 : $16568 \mathrm{ha}$, soit 3,34\% du territoire départemental. Classé en 1950, il s'étend sur les terres de neuf villes et communes.

6o. Forêt de formation spontanée et auto-régénérée appelée couramment forêt " primaire » (genseirin 原生林). Selon Ino.ue Susumu 井上曋 (professeur à l'Institut de recherche sur les forêts de l'université de Kyūshū, Kyūshū daigaku nōgakubu fuzoku enshū-rin 九州大学農学部附属演 習林 situé à Sasaguri), cette forêt se serait conservée «telle quelle, par auto-régénération, depuis l'époque Jōmon (Néolithique)». 


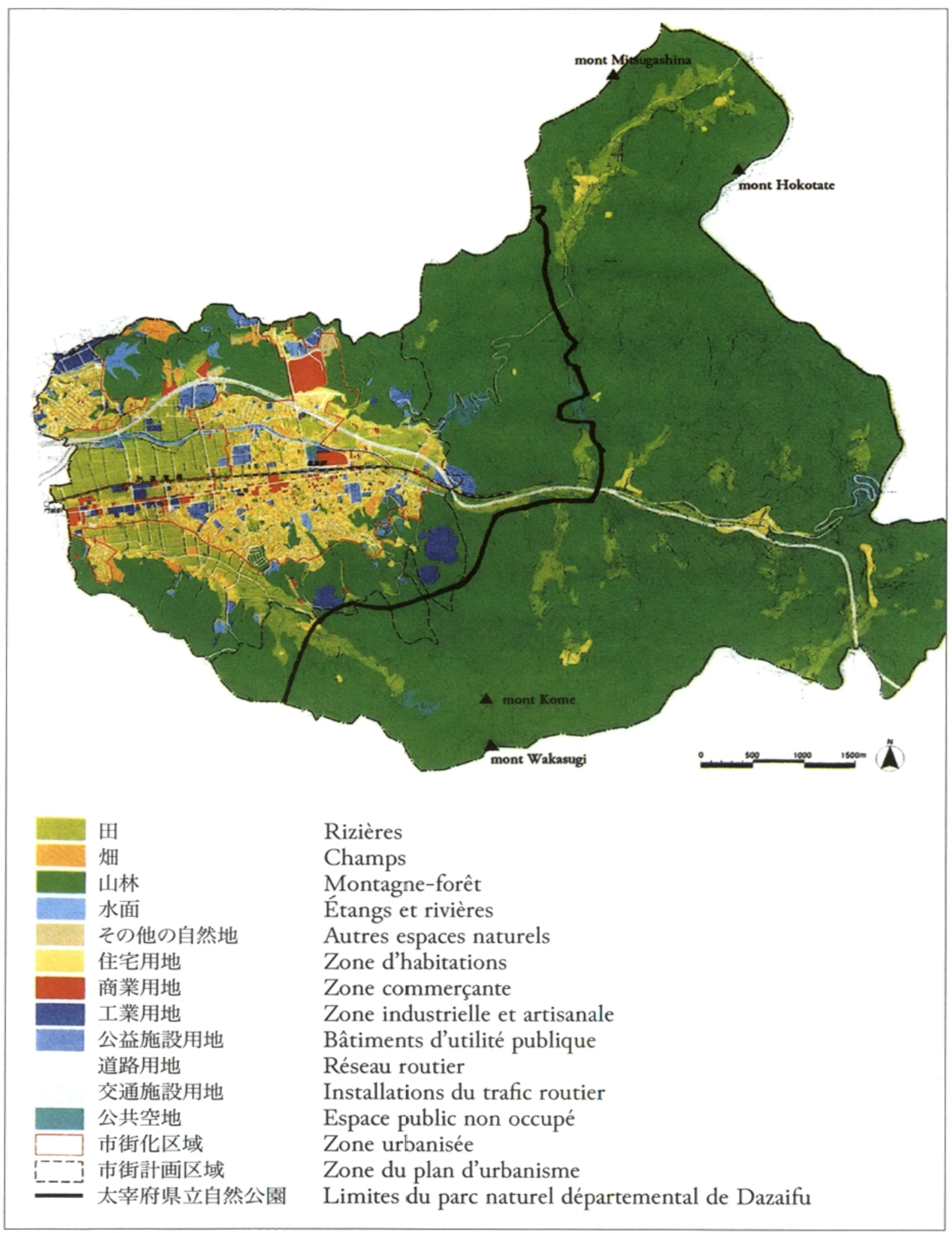

Carte 3 : Types d'occupation des sols. (Sasaguri-chō kikaku kōhōka 策栗町芥旧/伭 鈼課, 2003. Green Oasis Sasaguri. 2003 Dai niji Sasaguri-chō kokudo riyō keikaku 第. 次策栗町国 $\mathrm{t}$ 利用計曲, Sasaguri：19.) 


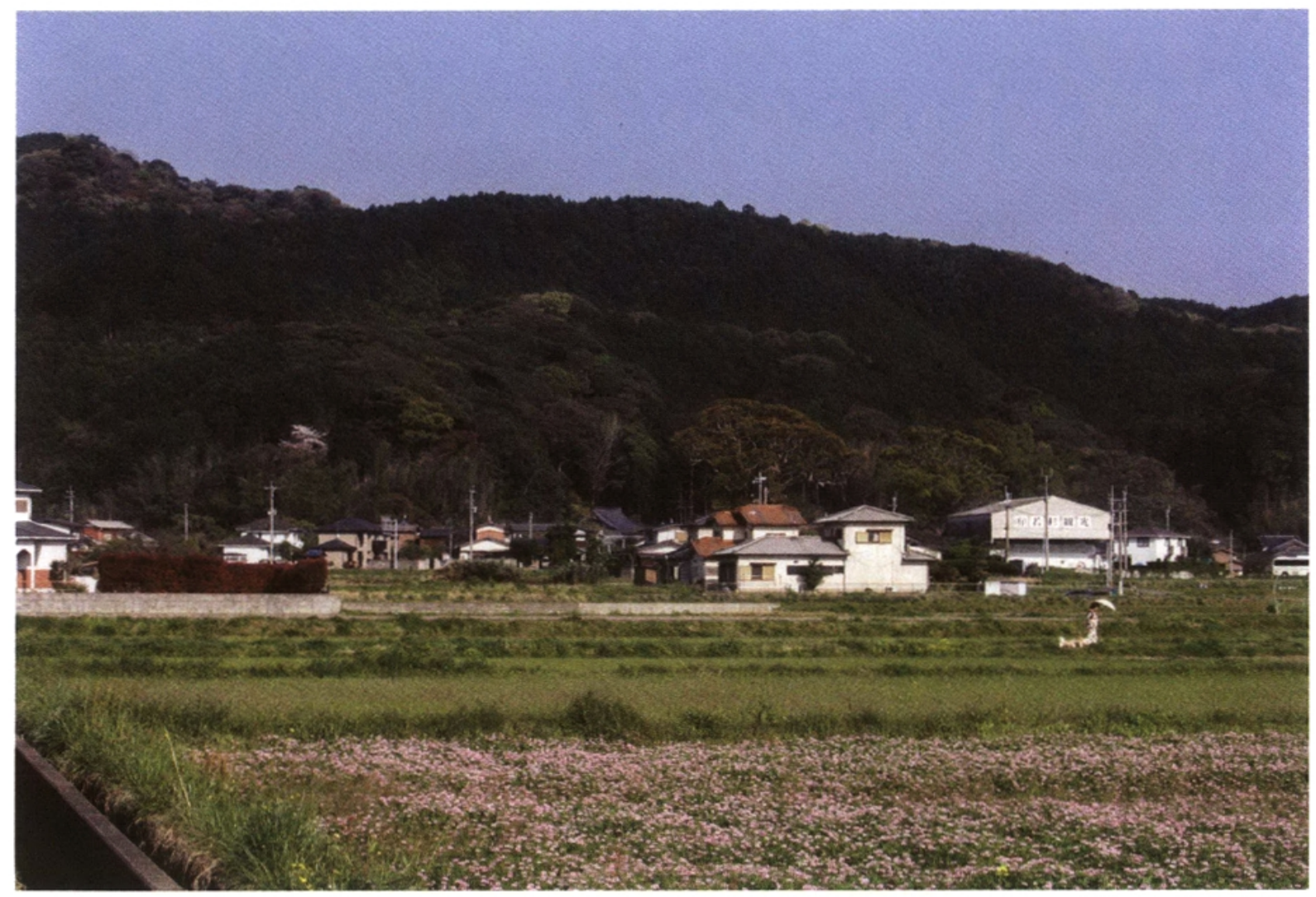

Fig. 2 : Forêt auto-régénérée la plus ancienne de Sasaguri - arbres sempervirents de la laurisylve à Kana.ide : massif forestier à gauche sur la $\mathrm{I}^{\mathrm{re}}$ ligne de colline ; surplombée par le sommet de la Montagne du dessus plantée en cryptomères ; au fond à gauche forêt de feuillus sur le mont Iimori. En bas à droite : la cime des deux camphriers de Tenjin. (Tous les clichés sont de l'auteur.)

principaux sont les châtaigniers à feuilles luisantes — sudajii スダジイ (Castanopsis sieboldii), tsuburajii ツブラジイ (Castanopsis cuspidata) (fig. 3), et de monumentaux camélias (yabutsubaki ヤブツバキ, Camellia japonica) et chênes à feuillage persistant (arakasbi アラカシ, Quercus glauca) (300 à 400 ans pour les plus anciens), associés au bas des pentes à des lauracées — tabunoki タブノキ (Machilus thunbergii). Dans l'enceinte du sanctuaire Hagio jinja 萩尾神社 à Haginoo poussent d'autres peuplements spontanés dont l'âge est évalué entre 400 et 250 ans : des persistants - isunoki イスノキ (Distylium racemosum) (fig. 4), mocbinoki モチノキ (Ilex integra), kuroganemochi クロ ガネモチ (Ilex rotunda), urajirogashi ウラジロガシ (Quercus salicina) — et des arbres à feuilles caduques — zelkovas du Japon (keyaki ケヤキ, Zelkova serrata), érables du Japon (irobakaede イロハカエデ, Acer palmatum) 一, qui côtoient quelques arbres plantés plus ou moins récemment. Cet ensemble végétal qui entoure les bâtiments cultuels forme ce que l'on appelle la forêt du sanctuaire (shasōin 社䣘林), liée à l'origine même des lieux de culte dédiés aux divinités. Des arbres peuvent y être plantés, mais ceux qui y poussent ne sont ni coupés ni taillés ${ }^{61}$. Cette " forêt » peut être réduite à quelques

6r. C'est le cas d'une majorité de sanctuaires au Japon. Mais la forêt de certains d'entre eux est exclusivement le résultat de plantations. Ainsi, celle du sanctuaire Meiji (Meiji jingū 


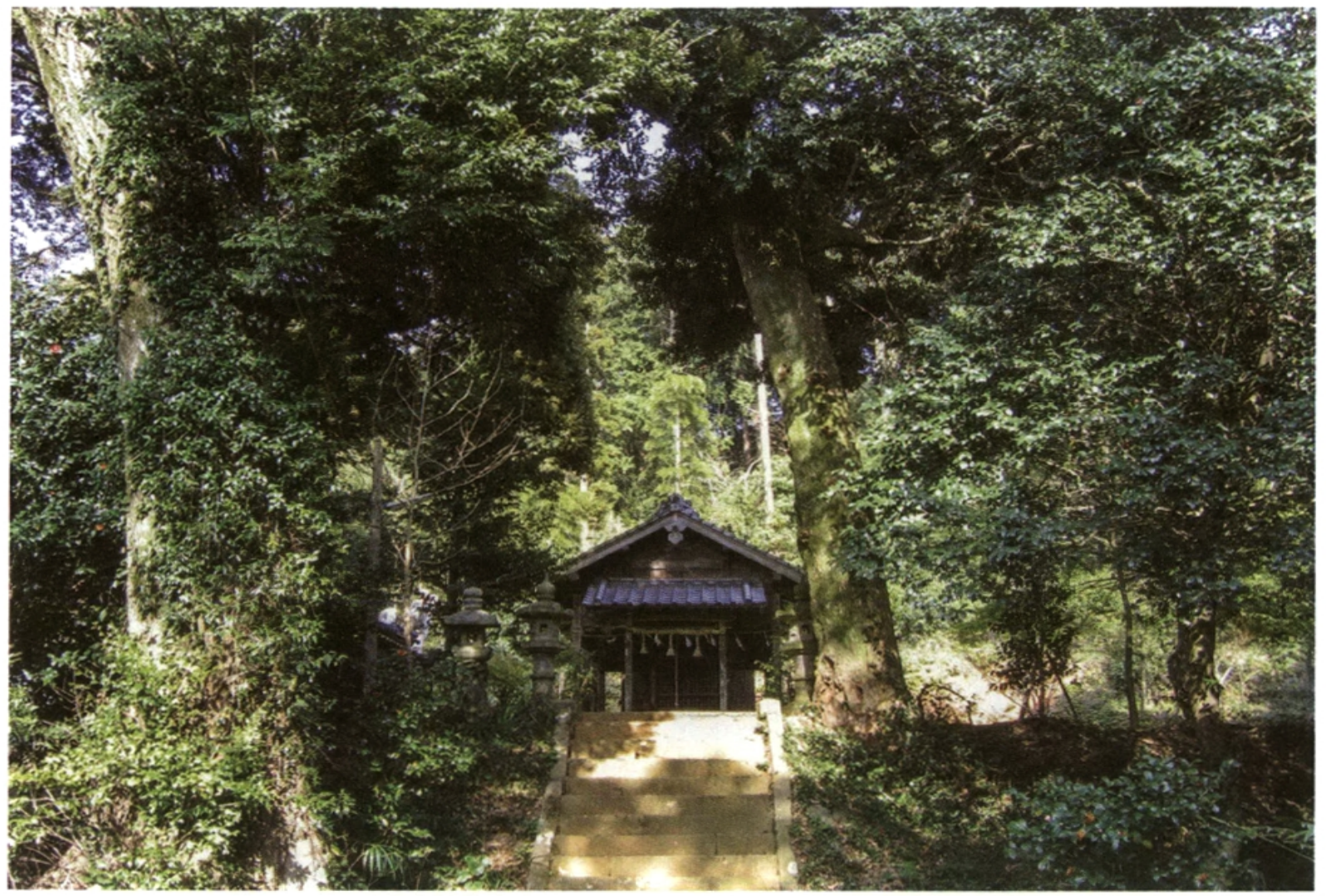

Fig. 3 : Châtaigniers à feuilles luisantes : sudajii (Castanopsis sieboldii) et tsuburajii (Castanopsis cuspidata) dans l'enceinte du sanctuaire Hōman-gū (Kana.ide).

arbres (ainsi la « forêt de Tenjin » de Takata), dont certains sont remarquables pour leur taille et leur âge, comme les deux camphriers géants (Cinnamomum camphora) (plus de $9 \mathrm{~m}$ de circonférence, $30 \mathrm{~m}$ de haut ; âge estimé entre 300 et plus de 400 ans pour l'un, 500 et I 000 ans pour l'autre) (fig. 5) du sanctuaire Oimatsu 老松神社 (Onaka) dont l'enceinte porte le nom de «forêt d'Ikuta » (Ikuta no mori 生田森), et même à un ou deux, comme les deux camphriers géants de l'ancien sanctuaire Tenjin 天神社 de Kana.ide (fig. 6) ${ }^{62}$. Toutes ces forêts de sanctuaires se trouvent aujourd'hui incluses dans les zones urbanisées ${ }^{63}$. En 1954 et 1994, les deux camphriers du sanctuaire Oimatsu ont été classés « monuments naturels » (tennen kinen butsu 天然記念物) de Sasaguri, ainsi que, en 2006, les deux forêts du Hōman-gū et du Hagio jinja (en tant

明治神宮) située en plein cœur de Tōkyō compte aujourd'hui 150000 arbres ( 246 espèces), qui ont été plantés sur les 70 ha de l'enceinte à partir de 1915, et dont beaucoup sont des espèces de la laurisylve. Aujourd'hui cette forêt est dite "artificielle-naturelle ", car elle a atteint un stade d'auto-régénération. Ce sanctuaire central du shintō moderne est particulièrement intéressant, car il est le contre-exemple d'un discours du shintō (actuel) revendiquant l'exemplarité de son rôle d'agent d'une continuité végétale "naturelle " immémoriale, grâce au culte de la « nature ", qui serait à l'opposé de l'évolution des religions ct civilisations occidentales centrées sur l'artificiel.

62. Sanctuaire réuni au Hōman-gū en 1926.

63. L'endroit où se trouve le sanctuaire d'Oto.inu, Hachiman-gū 八幡宮, est appelé «arbre divin " (神木 prononcé Jin-no-ki). 


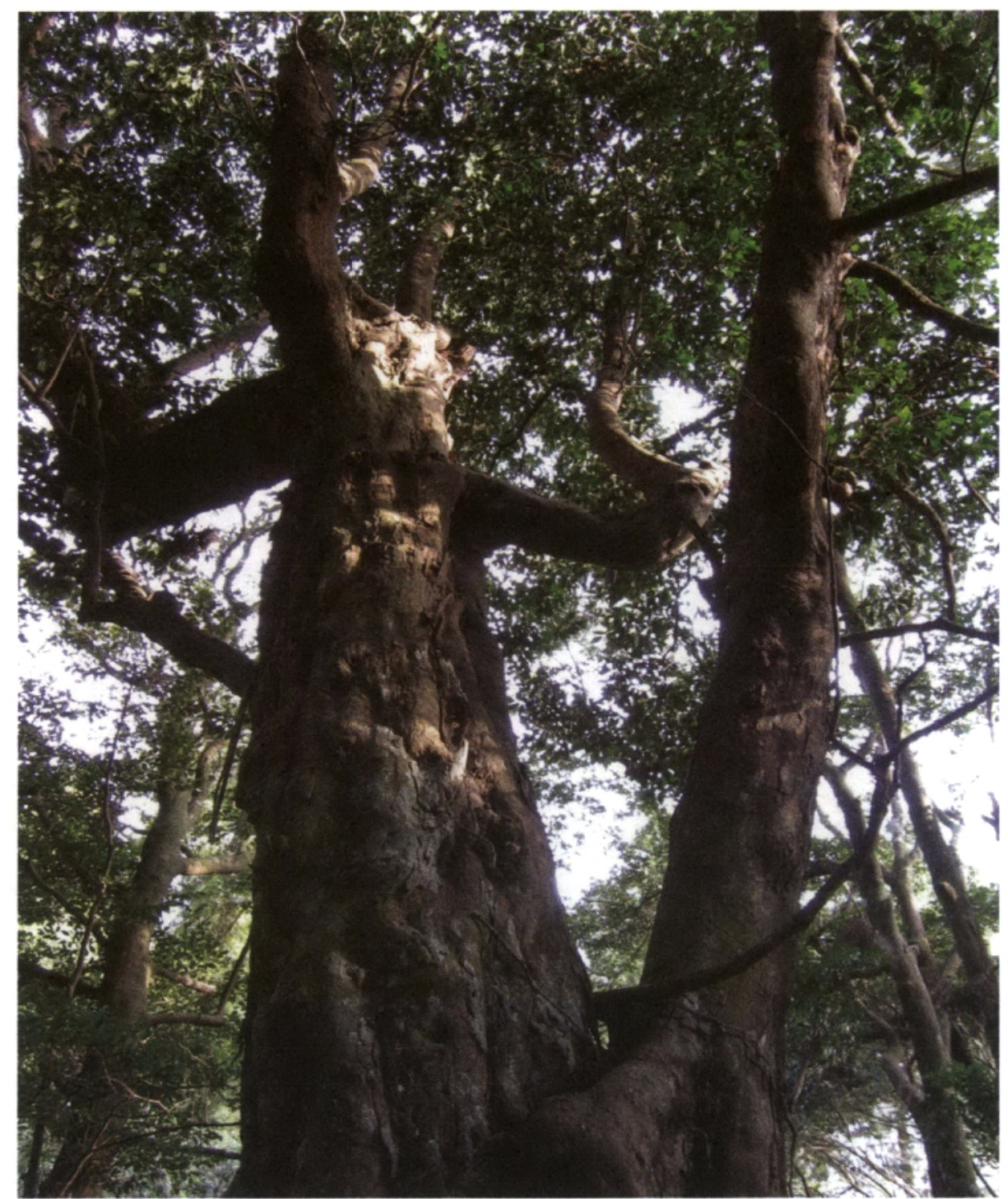

Fig. 4 : Peuplement spontané, isunoki (Distylium racemosum), dans l'enceinte du sanctuaire Hagio.

que milieux sylvestres anciens quasiment disparus du pays) et les deux camphriers de Tenjin pour leur taille et leur âge ${ }^{64}$.

D'autres petits espaces de forêts auto-régénérées de la laurisylve et d'arbres à feuilles caduques existent à l'état dispersé sur le territoire communal. À la différence des forêts de sanctuaires qui n'ont pas été l'objet d'usages coutumiers, ce deuxième type de forêts auto-régénérées est le résultat actuel de coupes régulières anciennes pour les diverses utilisations du bois (combustible, etc.). Ces parties boisées sont dites " naturelles » (tennen rin 天然林), c'est-à-dire non plantées. Elles ont repoussées naturellement après ces coupes et se présentent sous la forme de petits ensembles forestiers disséminés, de

64. «Camphriers-époux » qui auraient « un millier d'années » selon l'inscription posée sur les lieux. 


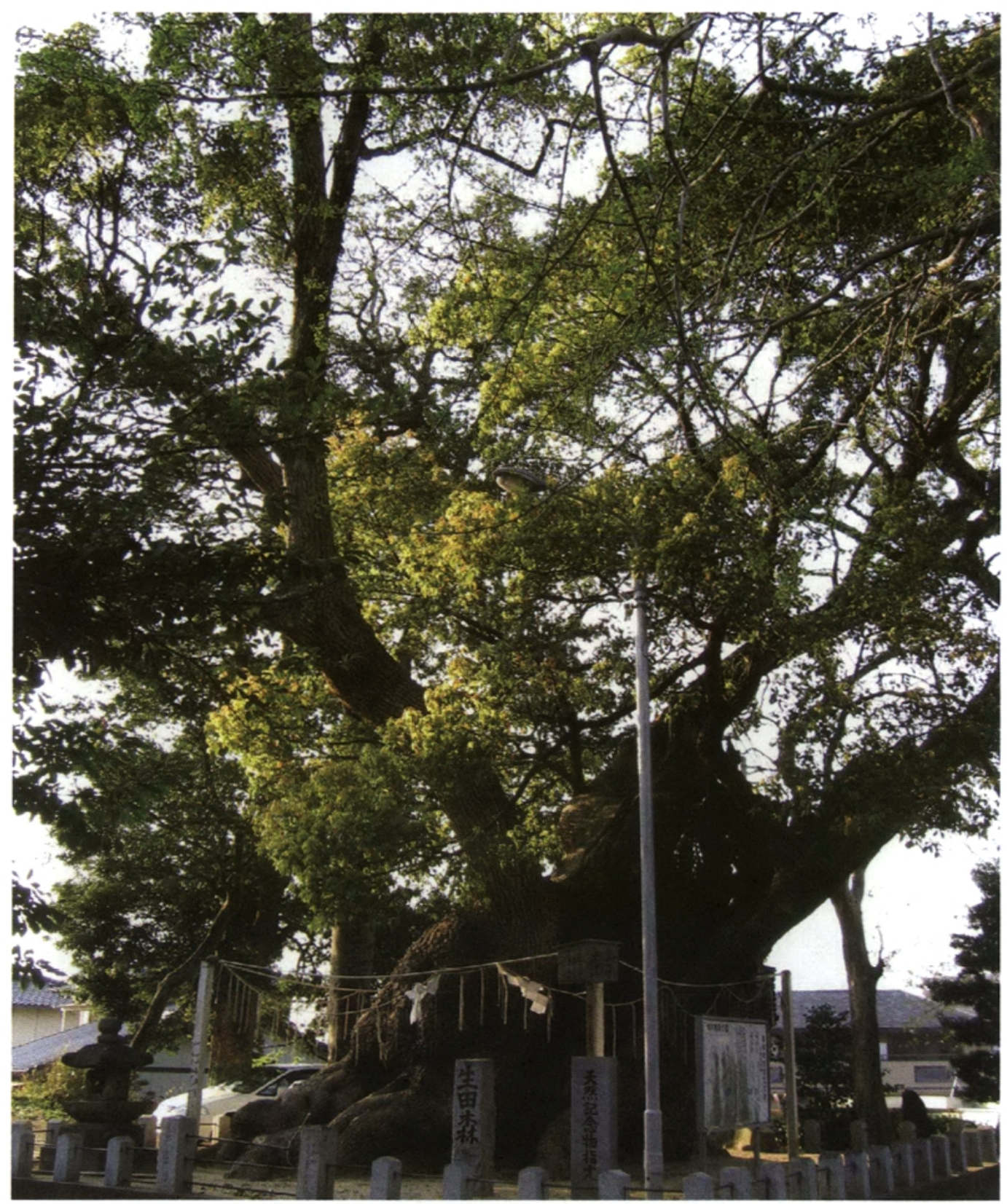

Fig. 5 : L'un des camphriers géants (Cinnamomum camphora) du sanctuaire Oimatsu (Onaka).

groupes de quelques arbres, ou même d'arbres isolés. Ces peuplements se situent soit sur des pentes peu élevées dans la continuité des secteurs habités (c'est le cas notamment d'une partie, environ un tiers, de la forêt ${ }^{65}$ de l'Institut de recherche sur les forêts de l'université de Kyūshū au nord des quartiers de Wada, Tsubakuro, Takata, Kana.ide), soit plus à l'écart le long de la vallée de la rivière Narubuchi 鳴㴊川, ou même au cœur des forêts plantées (Wakasugi, Yamate, Kido). On y retrouve, entre autres :

65. $482 \mathrm{ha}$, entre $50 \mathrm{~m}$ et $500 \mathrm{~m}$ d'altitude, à cheval sur les communes de Sasaguri et Hisayama. 


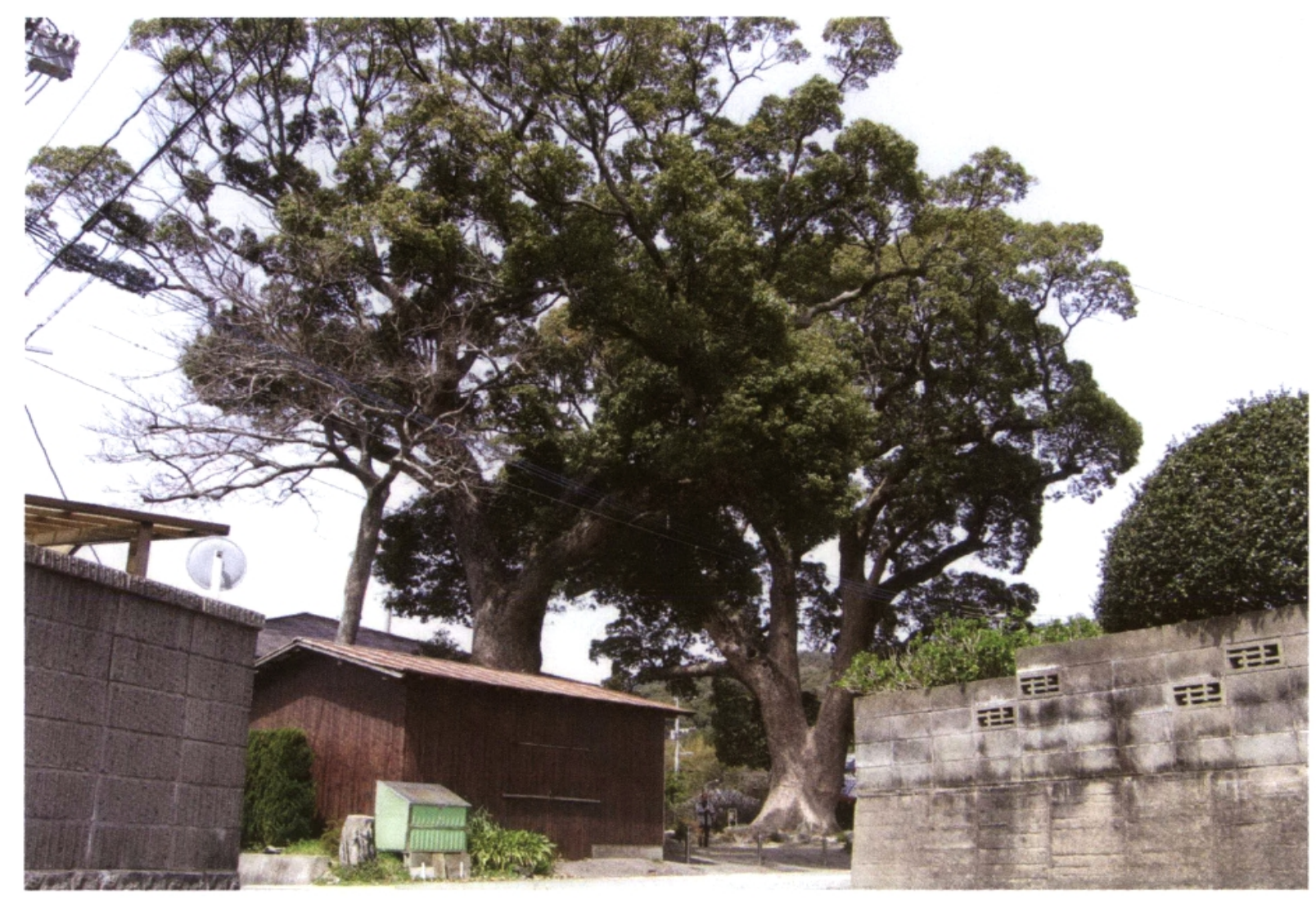

Fig. 6 : Les deux camphriers géants (Cinnamomum camphora) de l'ancien sanctuaire de Tenjin (Kana.ide).

- sur les pentes, les diverses espèces à feuilles persistantes de la laurisylve : châtaigniers (sbii シイ/椎, Castanopsis), chênes (kashi カシ/楅, Quercus), plusieurs espèces des familles des camphriers, des lauracées et des camélias (tsubakiツバキ/椿, Theaceae), des troènes du Japon (nezumimocbi ネズミモチ, Ligustrum japonicum), des aucubas ;

- surtout sur les crêtes, des arbres à feuillage caduque : des familles des Betulaceae (inusbide イヌシデ, Carpinus tschonoskii), des chênes (konara コナラ/小梅, Quercus serrata), des châtaigniers du Japon (Castanea crenata) ;

- et même une végétation de type maritime : taimin tacbibana タイミンタチバナ (Rapanaea neriifolia), pins rouges (akamatsu アカマツ/赤松, Pinus densiflora), pêchers de montagne (yamamomo ヤマモモ, Myrica rubra), plantes de bord de mer et chênes à feuilles caduques (mizunara ミズナラ, Quercus crispula) (Kana.ide, près de l'actuel Centre éducatif départemental $)^{66}$.

- Les plantations forestières ou “forêts artificielles" (jinkōrin 人工林)

Il en existe deux sortes à Sasaguri, de conifères et de feuillus, que l'on peut classer par ancienneté de plantation. Les plantations de conifères occupent actuellement

66. Fukuoka-kenritsu shakai kyōiku sōgō centā 福岡県立社会教育総合センター. Sasaguri-chō bunkazai senmon-iinkai, éd., Sasaguri chōshi. Minzoku-ben, p. 37 I. 
la majorité des surfaces boisées, couvrent surtout les sommets et hauteurs, et sont constituées de cryptomères sugi 杉 (Cryptomeria japonica) et de cyprès du Japon binoki 檜 (Chamaecyparis obtusa) (fig. 7$)^{67}$. Les premiers sont majoritaires dans toutes les zones forestières au sol riche et bien irrigué au sud de la rivière Tatara (Wakasugi, Sannō, sud de Yamate, sud de Kido, Naijū), les seconds sur les pentes moins riches au nord de la rivière (est de Kana.ide, Haginoo, nord de Yamate, nord de Kido).

C'est sur le mont Wakasugi que se dressent les plus anciennes forêts de cryptomères de la commune. Nombreux sont les habitants de Sasaguri pour qui ces arbres sont d'origine «naturelle». Néanmoins, selon les recherches les plus récentes ${ }^{68}$, à Kyūshū, à l'exception de la seule forêt primaire de cryptomères de l'île de Yaku 层久島 (yaku-sugi 屋久杉) ${ }^{69}$, et d'une forêt auto-régénérée de Miyazaki 宮崎, et même dans tout l'archipel japonais, il n'en existerait quasiment aucune qui ne doive rien à la main de l'homme ${ }^{70}$. La plantation se fait par bouturage des branches que l'on a mises préalablement en terre pour qu'elles fassent des racines. L'ancienneté de cette pratique trouve un écho dans la légende fondatrice éponyme du mont Wakasugi. Selon celle-ci, dans l'Antiquité, au retour de sa victoire sur les Trois royaumes de Corée, l'impératrice Jingū 神功皇后71 aurait transplanté sur cette montagne, en signe de reconnaissance envers les dieux, une branche de cryptomère du sanctuaire de Kashii香椎神宮 (Fukuoka) ${ }^{72}$.

67. Pour comparaison : $53 \%(13383383,04 \mathrm{ha})$ de la superficie forestière totale japonaise (25096947,48 ha) sont des forêts auto-régénérées, et $41 \%$ (ro346672,50 ha) des forêts plantées, qui sont constituées pour $44 \%$ d'entre elles de cryptomères et pour $25 \%$ de cyprès (Shinrin shigen no genkyō 森林資源の現況, 2007, site Rin.yachō.

68. Notamment celles de Ino.ue Susumu, professeur à l'institut de recherche de la faculté d'agronomie de l'université de Kyūshū à Sasaguri, qui a mené de nombreuses enquêtes à Kyūshū et dans toute la commune.

69. Unique au Japon, et inscrite au patrimoine mondial dcpuis 1993 pour ses arbres de plus de 2000 ans.

70. Miyajima Hiroshi 宮島筧, Nibon no tennen sugi 日本の天然スギ, texte non publié de la conférence au Symposium “Yakushima de katarō “Nihon no sugi” 》屋久島で語ろう:日本の杉 (26 novembre 1994), Yaku-chōritsu Yaku-sugi shizen-kan 屋久町立屋久杉自然館, p. II.

71. Jingū est mentionnée dans les Annales du viIr siècle (le Kojiki $i$ 古事記 et le Nibon shoki 日本㫪紀) comme épouse du quatorzième empereur Chūai 伸衰天皇, et mère du quinzième Ōjin 応神天皇. Mais son existence, située au III ${ }^{\mathrm{c}}$ siècle, a fait l'objet de nombreux débats et controverses entre les historiens.

72. Telle est la version la plus ancienne de l'histoire de fondation de Wakasugi. Elle est contenue dans le Hachiman Usagū On-takusenshū 八幡宇佐宫御託官集 du XII' siècle (Shigematsu Akihisa 重松 明久, éd., Hachiman Usagū On-takusenshū, Tōkyō Gendai shinchōsha 現代思潮社, 1986, p. 89), le document le plus ancien relatant ce partage : "Le bodhisattva Sainte mère [Jingū] ... partagea et planta le cryptomère du sanctuaire de Kashii (lieu où Jingū est vénérée) sur une haute montagne, elle y installa le Grand Ancêtre [Taiso, c'est-à-dire Izanagi 伊倠榙]. C'est la montagne appelée aujourd'hui

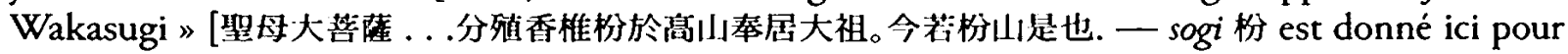
désigner le cryptomère, sugi 杉]. Cette version est aussi retenue au Xvir siècle par Kaibara Ekiken 貝原 益軒 dans le Chikuzen no kuni zoku fudoki (Kaibara Ekiken, éd., 1977, p. 403) et au XIX` par Aoyagi Tanenobu dans le Cbikuzen no kuni zoku fudoki shüi. Mais les autres histoires des origines écrites sous l'autorité du sanctuaire de Wakasugi inversent la légende en faveur de ce dernier : l'impératrice aurait transplanté dans l'enceinte du sanctuaire de Kashii une branche de cryptomère du mont Wakasugi (document le plus ancien : Cbikuzen Kasuyagun Wakasugi Taiso jinja engi 筑前糟屋郡分 


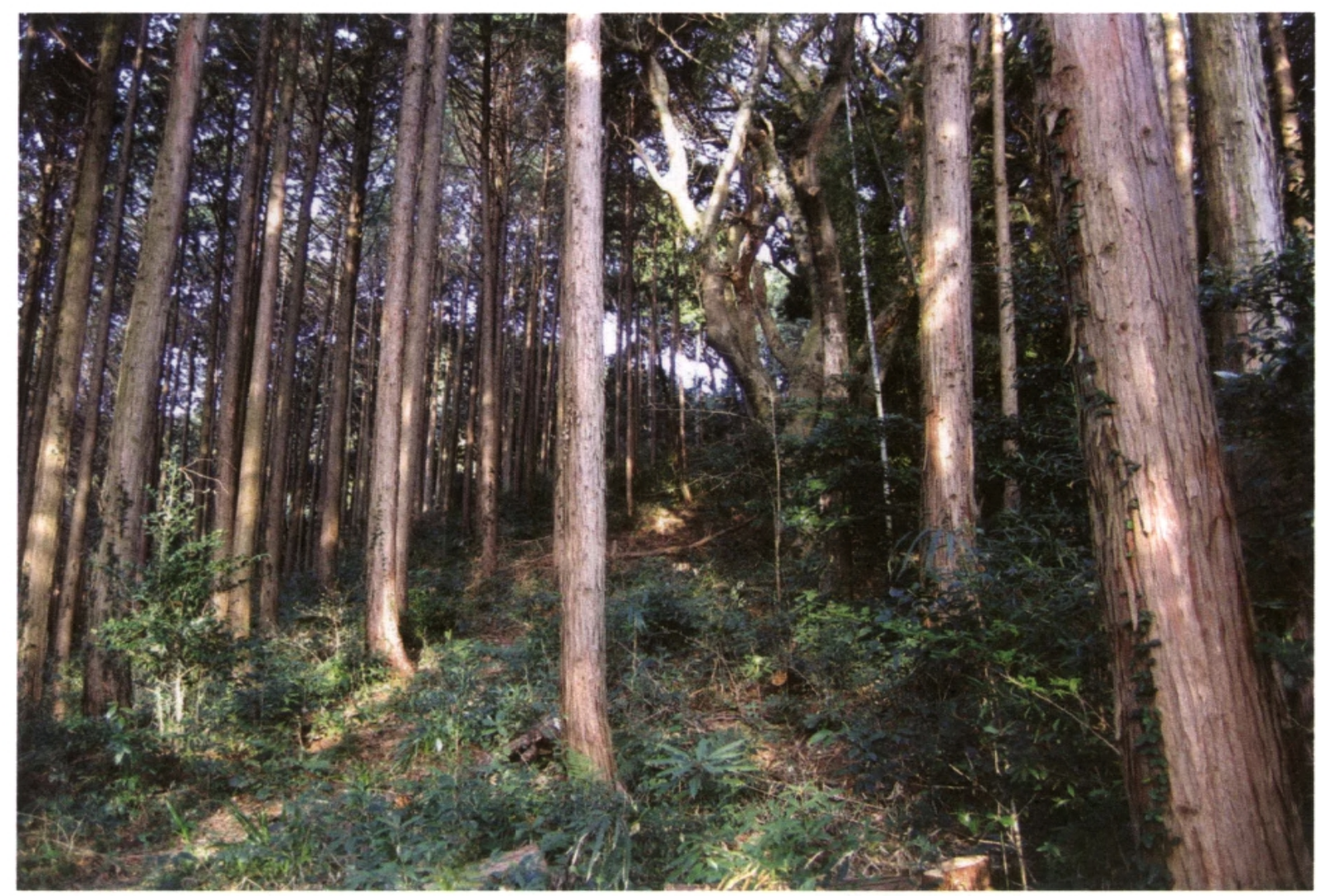

Fig. 7 : Plantation de cyprès du Japon (Chamaecyparis obtusa) (Kana.ide).

Cette bouture ayant donné un bel arbre, elle aurait reçu le nom d'aya-sugi 綾杉73, " cryptomère de belle forme ». De cette branche " partagée », viendrait en fait le nom originel de la montagne, Wake-sugi 分杉 "Cryptomère partagé ", dont l'altération aurait donné ultérieurement le nom actuel Waka-sugi 若杉 “Jeune cryptomère ». Cet

杉大祖神社縁起 [Genroku 元禄 5, 1692]. Kyūshū rekishi shiryōkan 九州歴史資料館, éd., Cbikuzen Kasuya. Wakasugi yama no bukkyô iseki 筑前粕屋:若杉山の仏教遗跡, coll. « Kyūshū no jisha shirīzu ” 九州の寺社シリーズ 8, 1986, vol. 8, p. 38-44). Cette inversion reflète la construction des rapports de pouvoirs entre les sanctuaires. La primauté de l'arbre et la transplantation secondaire sont prises comme un rapport de dépendance entre deux structures de culte. Il s'agit donc pour les auteurs des textes de placer en premier celle dont ils relèvent. Mais dans les deux cas, ce qui retient l'attention ici, c'est la mention de la plantation d'une branche (bouture) du cryptomère d'origine. Des extraits de tous les textes concernant les origines de Wakasugi ont été réunis dans Cbikuzen Kasuya. Wakasugi yama no bukkyô iseki, p. 35-49). Voir à ce sujet, dans ce même volume, les analyses de Mori Hiroko, "Le mont Wakasugi, son sanctuaire et le shugen dans l'histoire de Kyūshū et de l'Asie de l'Est ", p. 211-213, et de Suzuki Masataka, «Continuités et transformations de la société locale », p. 42-43.

73. Cryptomeria japonica var. araucarioides. L'une des espèces de cryptomères de Kyūshū. Aujourd'hui un seul cryptomère appelé « aya-sugi » se dresse à proximité du sanctuaire. Mais il n'appartient pas à cette espèce. Néanmoins, l'endroit où il se trouve est précisément celui que la tradition locale appelle " champ d'aya-sugi » ou «vallée des aya-sugi », là où Jingū aurait planté le premier cryptomère. Nishi Nihon bunka kyōkai 西日本文化協会, éd., Fukuoka-ken chiri zenshi 福岡県地理全誌 1, Fukuoka-ken-shi 福岡県史料, vol. 22, 1988, p. 466. 
acte est capital, car il fonde le culte du mont Wakasugi et l'histoire du sanctuaire, le Taiso-gū 太祖宮 qui se dresse sur son sommet (fig. 8). Cette légende reflète le fait que, antérieurement à toute construction, c'est l'arbre (planté ou préexistant sur les lieux) qui est la résidence première des divinités (ici, Taiso 太祖, le Grand Ancêtre, c'est-à-dire Izanagi, le père de tous les dieux). Et c'est autour de cet arbre que sont d'abord organisées les pratiques. Cet « arbre divin », shinboku 神木, entre dans la catégorie des supports ou reposoirs des dieux, appelée par les ethnologues yorishiro 依代74. C'est l'un des points d'articulation essentiels du végétal et du socio-culturel.

Les arbres de plusieurs centaines d'années sont concentrés dans la partie la plus haute de la montagne, c'est-à-dire dans l'enceinte du sanctuaire $(3,66 \mathrm{ha})$ au sommet (68I $\mathrm{m})$, le long du chemin d'accès et dans l'actuelle forêt domaniale $(87,83 \mathrm{ha})$ qui se situe juste en dessous, sur le côté nord (et se prolonge jusqu'à Satani sur le flanc sud). Les deux «Grands cryptomères » à côté du sanctuaire ont été classés « monuments naturels » de Sasaguri en 1978. Le périmètre de la forêt domaniale a fait l'objet de cinq enquêtes (2003-2009) qui ont permis de répertorier l'ensemble des cryptomères de plusieurs centaines d'années. Cent trente d'entre eux (entre $4 \mathrm{~m}$ et $16 \mathrm{~m}$ de diamètre) ont plus de 300 ans $^{75}$. Les plus remarquables par leur taille et leur âge (qui ont tous un ADN différent) ont reçu des noms, Grand cryptomère du Yamato (Yamato ōsugi 大和 大杉, 16,15 m de circonférence, $45 \mathrm{~m}$ de haut, dont l'âge est évalué à 700 ans) (fig. 9), cryptomère Aya (Aya-sugi, $6 \mathrm{~m}$ de circonférence, $20 \mathrm{~m}$ de haut) (fig. 10), cryptomère Fourche de Tōda (Tōda no futamata sugiトウダの二又杉, 6,91 m de circonférence, $35 \mathrm{~m}$ de haut, classé en 2000 par le Ministère des forêts comme l'un des cent arbres les plus grands du pays), cryptomère Qui joue (Jare sugi じゃれ杉, II m de circonférence, 25 $\mathrm{m}$ de haut), cryptomère aux Sept fourches (Shichimata sugi 七又杉, 9,8 $\mathrm{m}$ de circonférence, $20 \mathrm{~m}$ de haut). Les cryptomères du mont Wakasugi sont considérés comme des « arbres divins ». À ce titre la coupe en est, en principe, interdite. Nous verrons qu'en réalité, même dans cette zone, des coupes ont été effectuées au cours de l'histoire. En dehors de ces grands arbres du sommet, le reste de la montagne est planté de cryptomères (un tiers) et de cyprès (deux tiers), destinés - initialement - à être coupés et vendus.

Comme nous le verrons plus loin, les autres forêts de conifères (cryptomères, cyprès, pins) actuelles résultent de campagnes de plantations locales systématiques faites par les habitants à partir de la fin de XVII ${ }^{\mathrm{c}}$ siècle dans les secteurs montagneux : d'abord à Arata (Sannō) sur les pentes nord-est et sur les basses pentes du mont Wakasugi, sur le mont Kome (Sannō), puis dans la partie nord et nord-est de la commune (Kido, Haginoo, Kana.ide). Ces plantations, exploitées pour la vente du bois, furent régulièrement coupées, mais elles ont été constamment replantées et entretenues jusque dans la seconde moitié du $\mathrm{xx}^{\mathrm{e}}$ siècle. Une grande partie de ces forêts sont classées comme forêts de protection (保㚣林 boan-rin) pour la sauvegarde des ressources en eau et contre les glissements de terrain. Pourtant aujourd'hui, pour cause de dépréciation

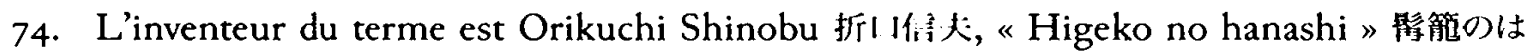
なし, dans Orikuchi Shinobu zenshü 折l.J倍夫全集, 1975, vol. 2 (Ic éd. 1915).

75. Cf. le rapport non publié de la cinquième enquête sur les cryptomères du mont Wakasugi (Kurose Shigefumi 黒瀨茂文, Wakasugi yama ósugi bunpu chōsa, daigoji, 茯杉川火スギ分布調杰. 第 5次, Sasaguri-chō kyōiku-iinkai 策要i町教育委員会, 2009). 


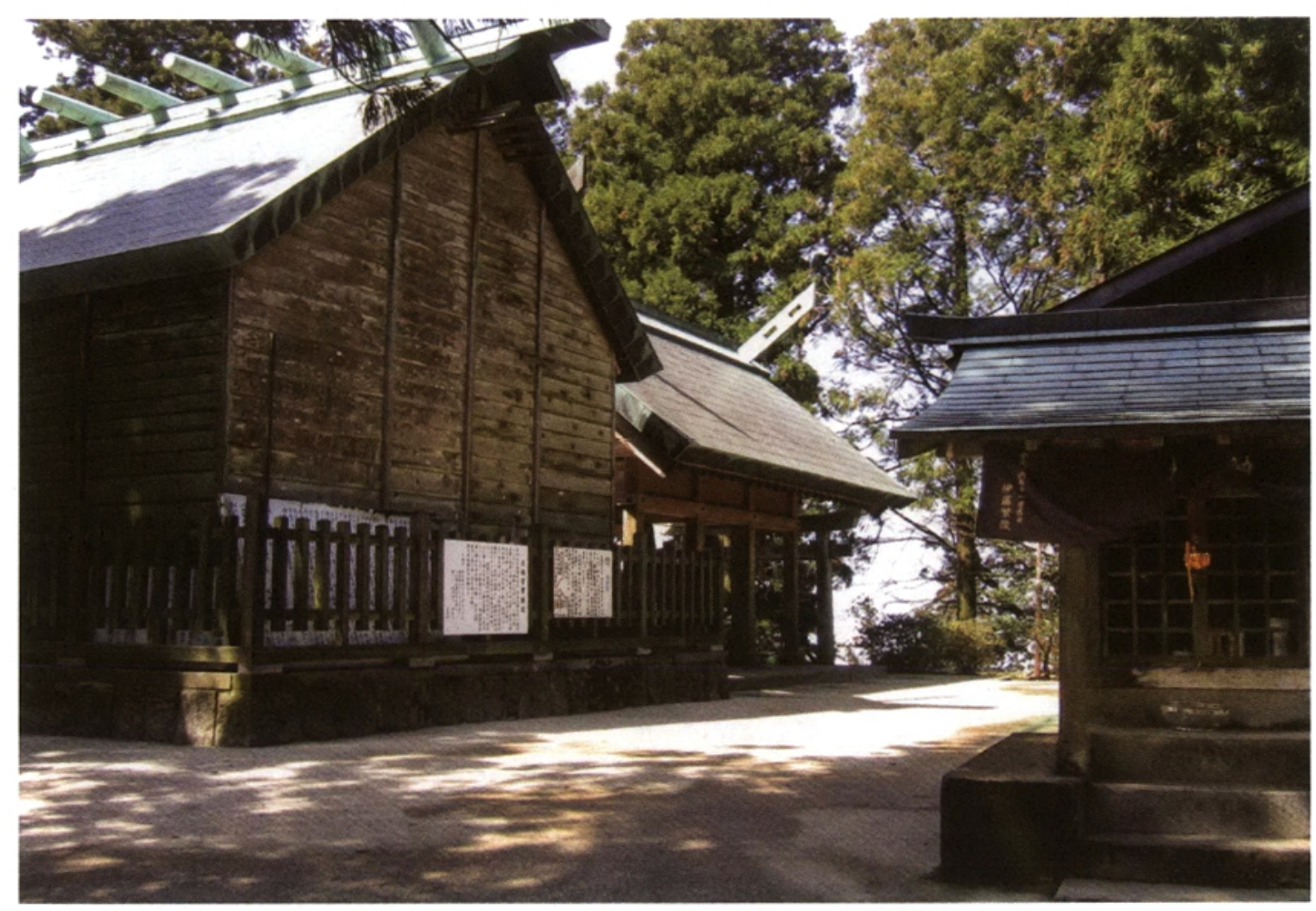

Fig. 8 : Le sanctuaire Taiso-gü sur le sommet du mont Wakasugi et ses cryptomères.

du matériau bois, toutes les forêts de conifères ont dépassé la date régulière de coupe et le manque d'entretien peut générer des nuisances, voire des catastrophes.

En dehors de ces zones, après la guerre, cryptomères et cyprès furent également plantés dans la zone intermédiaire entre le haut des montagnes et les villages, sur les espaces des anciens communaux, où les peuplements sont aujourd'hui de première ou seconde génération.

Le second type d'espaces plantés est celui des forêts de feuillus. Il faut noter un mouvement de replantation récent (depuis 2008) d'arbres à feuilles caduques sur les friches et anciennes coupes, initié par le département, qui reverse aux communes une partie de l'impôt sur l'environnement pour la gestion et la protection des forêts communales. En outre, des « forêts » de feuillus plantés (auxquels sont parfois associés des conifères) d'un type un peu particulier se rencontrent dans les principaux parcs de loisir de la commune. Six d'entre eux sont situés près des zones forestières, en bordure des routes d'accès aux quartiers périphériques : le Nouveau Yoshino (Shin Yoshino 新吉野) à l'est, le long des Sept virages de Gō-no-haru (Kido), où à la fin du XIX siècle (189o) furent plantés trois-cent-cinquante érables et mille cerisiers pour évoquer Yoshino (Nara) et ses mille cerisiers ; ainsi que d'autres parcs ouverts successivement depuis la seconde moitié du $\mathrm{xx}^{e}$ siècle : au nord, sur la route allant de Kana.ide à Haginoo, le parc de Kannon 観音公園 (cent pruniers, deux cents cerisiers, deux cents azalées) ; dans la vallée Narubuchi, le parc au-dessus du barrage du même nom (cerisiers, chênes, 2002) ; au nord-ouest, une partie de la forêt expérimentale de l'Institut de recherche de Kyūshū (Takata, 367 ha à cheval sur les communes de Sasaguri et Hisayama) existant 


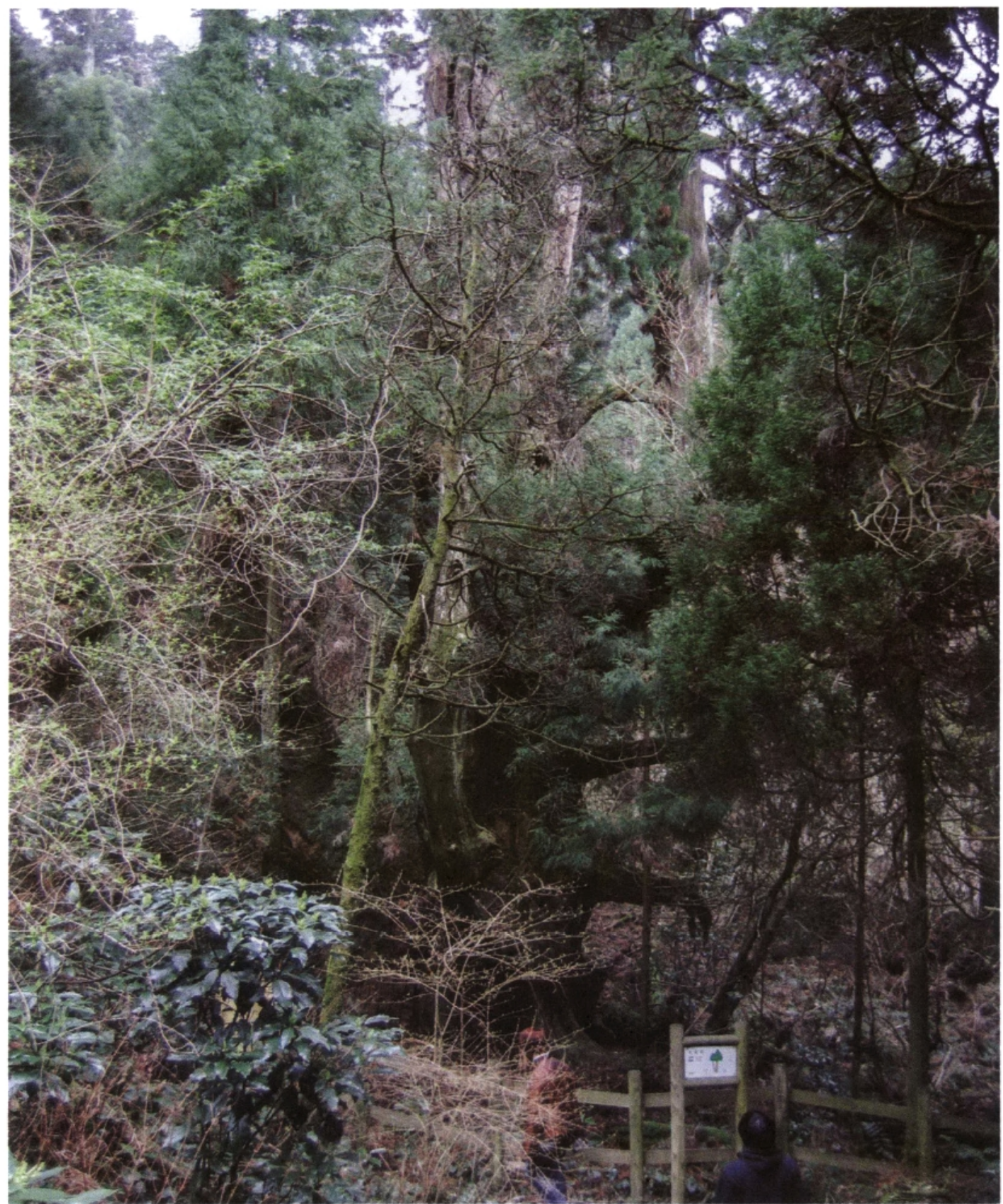

Fig. 9 : Grand cryptomère du Yamato (Yamato ösugi, 16,15 $\mathrm{m}$ de circonférence, $45 \mathrm{~m}$ de haut, dont l'âge est évalué à 700 ans).

depuis 1922 et ouverte au public depuis 2010, cogérée avec la commune ; au sud, le parc de Sasaguri 篠栗公園 autour du Dainichi-ji $\left(\mathrm{n}^{\circ} 28\right)$ où furent plantés des cerisiers, et, le parc des sports (dit Forêt du casque, Kabuto no mori 兒の森) à Wakasugi (cinq cents cerisiers, trois cents chênes, kunugi クヌギ/橡, Quercus acutissima), ouvert en 20Io. Enfin, en zone urbaine, le parc de Nishiura 西浦公園 (Onaka), où furent plantées plusieurs centaines d'azalées et de cerisiers. Dans cette ligne des parcs de feuillus, la Forêt de l'arboriculture (jugei no mori 樹芸の森, 47 ha s'étendant dans la montagne aux confins de Haginoo, Yamate et Kana.ide) créée en 1977 par le département puis reprise par 


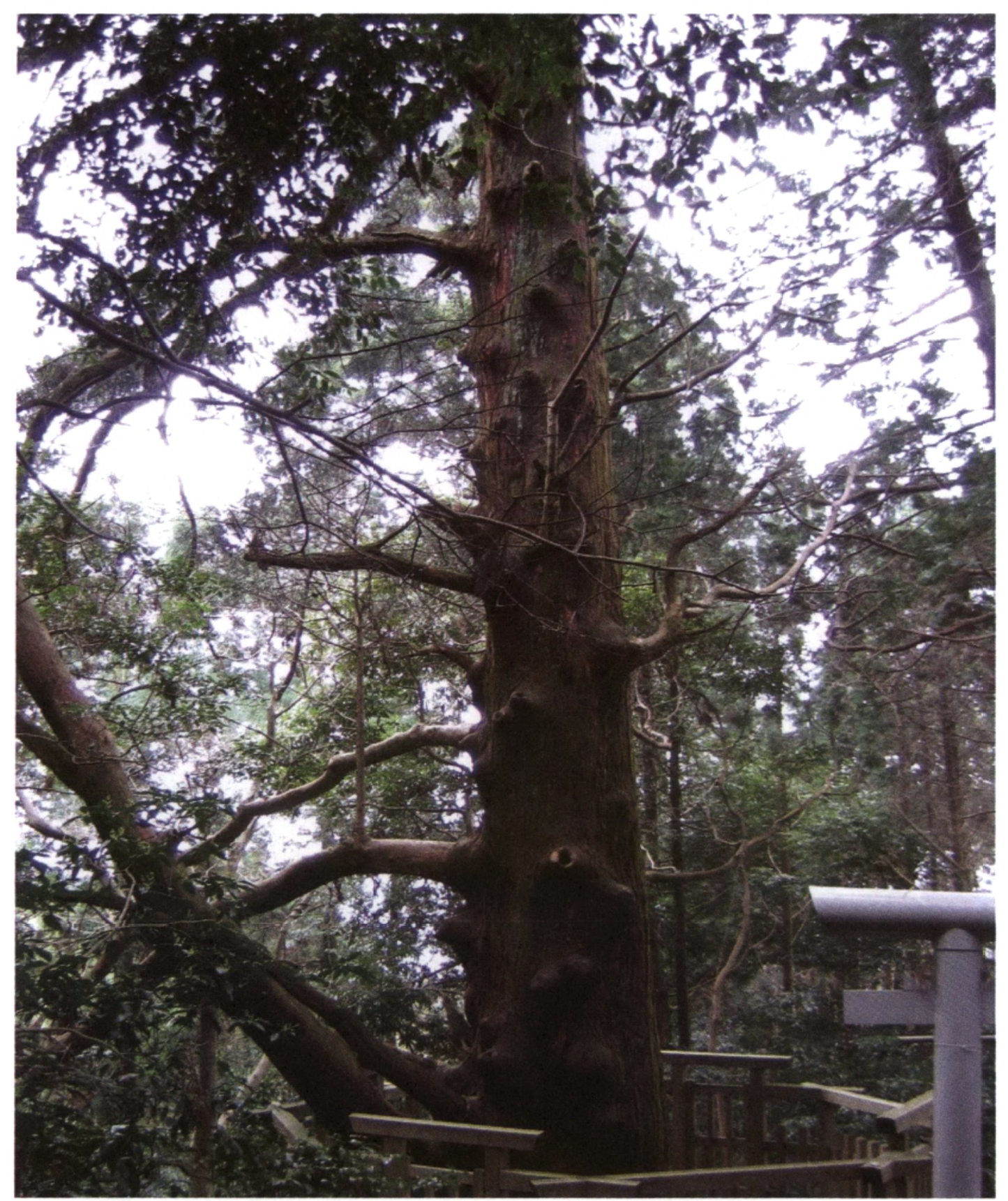

Fig. 10 : Cryptomère Aya (Aya-sugi, $6 \mathrm{~m}$ de circonférence, $20 \mathrm{~m}$ de haut).

la commune, est, à l'inverse, l'ouverture au public de la forêt de protection existante comme parc récréatif, avec structures communales de gestion et d'accueil.

- Les repousses sauvages

Une végétation de feuillus non entretenus et de broussailles s'est développée principalement sur les coupes faites dans les années 1980-1990 dans les montagnes non replantées à cause de la perte de valeur du bois, et sur les terres basses et intermédiaires abandonnées. 
Un autre type de végétation à prolifération rapide et néfaste, les bambous, a envahi les lieux laissés à l'abandon : certaines anciennes coupes non replantées, les bords des chemins (notamment ceux du pèlerinage, qui ne sont plus empruntés à la suite du développement de la pratique pérégrine en voiture) (fig. II) et les parcelles attenantes, ainsi que les anciens cimetières collectifs abandonnés. Il s'agit de bambous géants (mōsō chiku 孟宗竹, Phyllostachys edulis et madake 真竹, Pbyllostachys bambusoides) qui peuvent atteindre 25 mètres de haut et forment des forêts denses, sombres et inhospitalières, qui ne cessent de proliférer mais aussi de se dégrader (chutes des bambous morts, etc.) et d'entraver les végétations environnantes. Ces bambouseraies sont le paradigme même du délaissement et de l'ensauvagement des montagnes villageoises. Ce n'est que tout récemment qu'a été lancée un début de campagne locale de récupération et de nettoyage de ces espaces.

\section{- La déforestation}

Enfin, il faut noter qu'un certain nombre de forêts ont été détruites récemment, essentiellement dans la partie ouest de Sasaguri. C'est le résultat, d'une part, de l'ouverture des grandes voies routières et de l'urbanisation (structures scolaires et leurs stades, grands ensembles comme Bentana Hills ベンタナ・ヒルズ à Takata, grandes structures collectives comme le Centre départemental éducatif de Kana.ide, zones industrielles, artisanales ou commerciales), qui se sont intensifiées surtout entre les années 1980 et 1990, durant la période de prospérité pour ce type de constructions avant l'effondrement de la bulle économique. C'est aussi, d'autre part, la conséquence de l'implantation récente de vastes cimetières sans un arbre, gérés par des entreprises extérieures à la commune, appelés «parcs des âmes 》 (reien 霊園), notamment à Tsubakuro (Sasaguri reien 篠栗霊園, 81 $850 \mathrm{~m}^{2}$, et Gokuraku reien 極楽霊園, $29454 \mathrm{~m}^{2}$ ).

Ce simple panorama permet de voir en quoi les $6 \mathrm{I} \%$ boisés de ce territoire communal exigu ne forment pas « une forêt », mais sont constitués par quantité de milieux forestiers dont l'anthropisation est ancienne, diversifiée et continue, et dans lesquels de profonds changements sont survenus à une époque récente.

\section{Les usages : les montagnes-forêts comme ressources}

Pour les habitants, les milieux forestiers sont d'abord des espaces de ressources multiples. Mais leurs besoins, et donc les modalités d'utilisation, ont été considérablement bouleversés en l'espace d'un siècle et demi.

Actuellement, si l'on parle des «forêts » aux habitants, cela évoque immédiatement pour eux non pas la montagne-forêt du village (sato-yama) proche, mais ce qu'ils nomment la «montagne des terres du fond/reculées » (okucbi no yama 奥地の山) et les plantations de conifères, objet d'exploitation pour le bois de coupe destiné à la vente. Les activités qui sont liées à ces lieux sont aujourd'hui extrêmement réduites.

Ainsi, à Haginoo, presque tous les habitants (43 maisons) sont employés à l'extérieur du village. Seuls quatre d'entre eux continuent à entretenir leurs propriétés forestières de cyprès et de cryptomères, mais sans pratiquer de coupes d'éclaircies ou de détourage, uniquement en élaguant les branches basses et en nettoyant les broussailles autour des arbres. Et ceci, " seulement pour ma propre satisfaction 


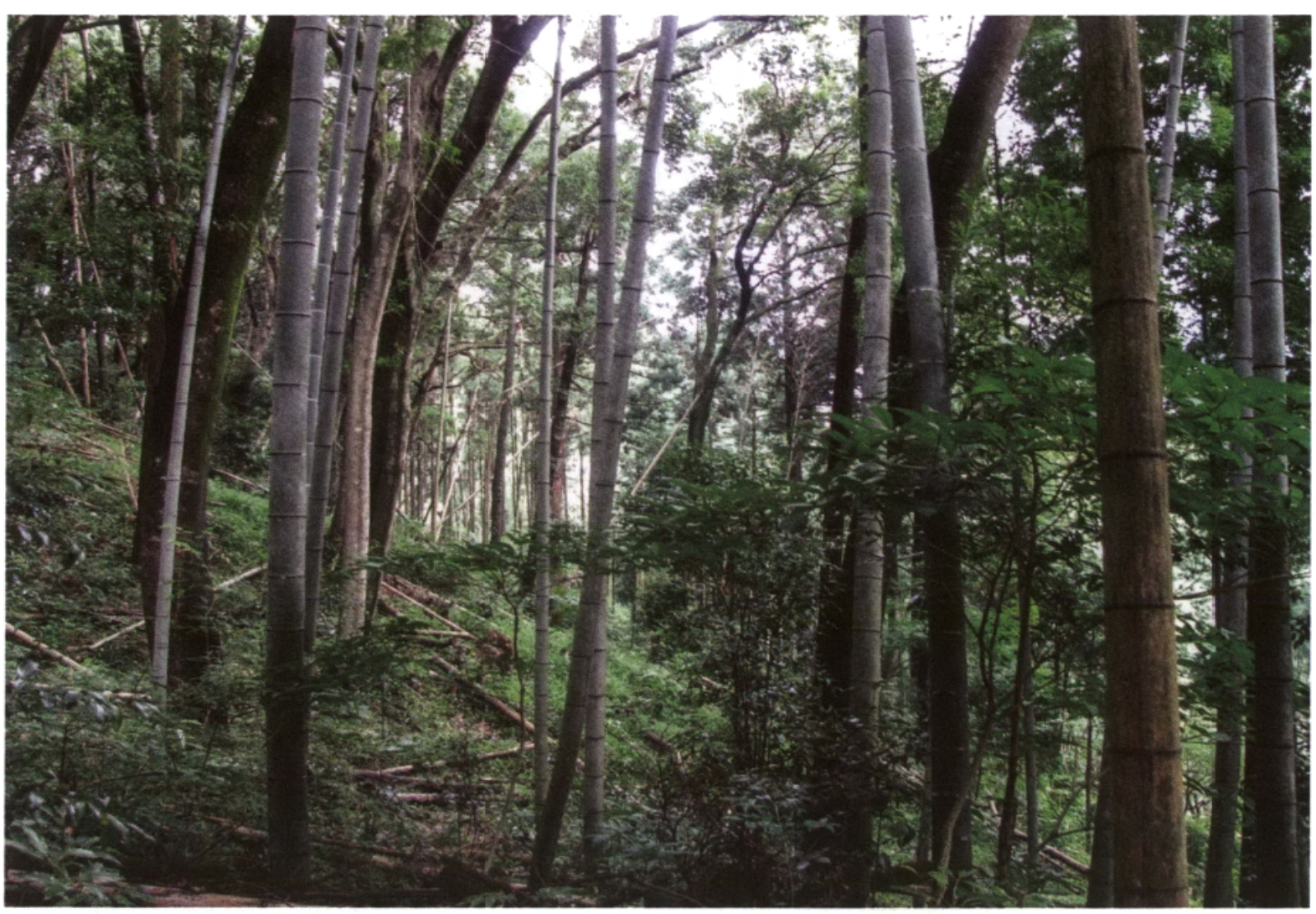

Fig. II : Bambous sur des terres non entretenues le long du chemin de pèlerinage (Naka-no-Kōchi).

esthétique et morale, pas pour l'argent 》, confie l'un d'eux, Matsuo Kenji 松尾健治 (né en 1940) qui possède une plantation de cyprès déjà ancienne (un siècle), «car, depuis les années 1990 le prix du bois étant très bas, personne ne fait de coupes pour la vente. Néanmoins, si un minimum d'entretien n'est pas fait, à l'avenir si les prix remontent, il n'y aura rien à vendre de correct. " Sur certains sommets, comme à Furuno 古野 ou sur le mont Mitsugashira, des repousses sauvages de feuillus ont remplacé les anciens bois de chênes (kunugi) utilisés autrefois pour le charbon de bois.

A Wakasugi également, presque tous les habitants travaillent aujourd'hui à l'extérieur, principalement à Fukuoka, faisant ainsi des allers et retours quotidiens. Mais, c'est collectivement, deux fois par an en été et en automne, à raison d'un membre pour chacune des 47 (anciennement 50) maisons, qu'ils font le nettoyage des herbes sous les plantations de cyprès et de cryptomères de la montagne. Jusque dans les années 1950, les parcelles entièrement coupées étaient replantées dès l'année suivante : un tiers en cyprès, deux tiers en cryptomères. Les arbres abattus entre février et mars pour éclaircir les plantations étaient alors vendus. Aujourd'hui ces coupes d'entretien sont faites par dix hommes du village, mais le bois est abandonné sur place. Non seulement ce bois n'est plus vendu, mais alors que toutes les réparations ou les constructions des bâtiments collectifs (sanctuaire, Oku-no-in 奥の院, Maison communale) étaient effectuées jusqu'au milieu du siècle dernier avec le bois de la montagne, aujourd'hui celui-ci est acheté à l'extérieur. 
Cette situation se retrouve dans tous les autres secteurs de Sasaguri (Arata, Yamate, Kido) où les habitants possèdent des forêts de conifères pour la vente mais ont renoncé à faire des coupes depuis la chute du prix du bois.

En plus de cette exploitation forestière des plantations de conifères (aujourd'hui plus ou moins suspendue), subsistent quelques usages locaux liés à d'autres peuplements végétaux. À Takata, par exemple, outre les plantations de conifères (trois quarts des forêts de ce quartier), une partie du quart forestier restant est constitué de plantations de chênes (kunugi) pour la culture du lentin des chênes (shiitake シイタケ, Lentinula edodes), champignon très valorisé et destiné à la vente. Certains pratiquent encore la collecte des végétaux sauvages comestibles, ou des plantes médicinales et des parties de feuillus à partir desquelles ils fabriquent des remèdes de la pharmacopée coutumière (ainsi les feuilles, branches et fruits des troènes du Japon, nezumimochi, récoltés dans les montagnes de Kana.ide et Yamate par un homme d'Arata).

L'usage des montagnes-forêts en tant que ressources matérielles est donc aujourd'hui extrêmement limité et majoritairement lié à la valeur et à l'exploitation commerciales de celles-ci en relation avec les conditions économiques extra locales. Mais ceci est loin d'avoir toujours été le cas. Si l'on interroge les habitants des villages sur ce qu'ils faisaient des montagnes-forêts au début du $\mathrm{xx}^{e}$ siècle et jusque dans les années 1960 , aussitôt ils insistent sur le fait que, pour eux, la montagne était avant tout le lieu où l'on trouvait alors un grand nombre des produits de première nécessité pour la vie quotidienne. Dans ce cas, ils parlent surtout de la montagne-forêt du village, à proximité des habitations, dont une grande partie est aujourd'hui remplacée par des plantations de conifères. Ils témoignent ainsi de ce que, en quelques décennies, les mêmes espaces ont été considérés et utilisés de façon très différente.

Cinq usages principaux de cette forêt proche apparaissent dans les discours et les documents locaux concernant l'histoire des pratiques. Jusque dans les années 1950, la montagne reste le lieu essentiel de l'accès aux ressources en fourrage, engrais, combustible, bois de construction et produits de subsistance ${ }^{76}$. Fourrage, engrais et combustible méritent une mention particulière pour l'importance des volumes prélevés. Les machines agricoles commencent à être utilisées à partir des années 1955 . Jusqu'à cette date, chaque maison vivant des travaux de la terre et de la forêt abrite au minimum un bovin et/ou un cheval. Les bovins étant utilisés pour tous les travaux agricoles et forestiers, les chevaux davantage pour les transports ${ }^{77}$. Aussi chaque village possède-t-il des « montagnes à herbes/fourrage » (kusa yama 草山 ou magusa yama

76. A titre d'exemple, en 1957 , les $904,5 \tan \left(\mathbb{x}_{\mathbf{x}}, \mathrm{I} \tan =991,7 \mathrm{~m}^{2}\right)$ soit environ 90 ha, de terres de Wakasugi étaient répartis en trois parts à peu près équivalentes entre : rizières 329 tan, champs secs $278 \tan$, forêts et landes $296,5 \tan$ (dont : bambouseraies $70,4 \tan$, forêts de pins $37,5 \tan$, plantations de cryptomères et de cyprès $116,2 \tan$, forêts de feuillus 72,4tan). (Gōya Takeshiro 合屋武城, Chikuzen Wakasugi kyōdoshi 筑前若杉郷土誌, 1957, p. 123).

77. André Leroi-Gourhan (Pages oubliées sur le Japon, 2004, p. 454), qui vécut au Japon de 1937 à 1939, décrit la situation du paysan japonais en des pages mémorables (dont certaines interprétations et prises de position doivent aujourd'hui être nuancées), où il note l'absence de toute machine (tracteur ou faucheuse) mais aussi de charrue en métal, les travaux étant faits avec "...des herses à dents de bois, des araires de bois, des houes, des bêches aux lignes antiques et incommodes, des binettes minuscules. " 
秝山), dites aussi 《lieu de fauchaison des herbes》(kusakariba 草刈り場), 《 montagne où l'on prend le fourrage » (kusatori yama 草取り山), où est coupé le fourrage et qui servent aussi de pâturages pour les animaux. $\mathrm{Si}$, pour une part, les engrais des champs et rizières sont constitués par le fumier animal et les matières de vidange, ils sont aussi faits d'« engrais vert 》 (ryokubi 緑肥), herbes et basses branches coupées provenant de ces mêmes lieux. Ces végétaux étaient foulés par les animaux avant d'être épandus.

Aux époques antérieures où l'autarcie était encore plus accentuée, la dépendance à l'égard de la montagne était identique et encore plus critique. À l'époque d'Edo, avant le repas du matin, chaque homme rapportait deux bottes de fourrage à la maison. Au début de Meiji (r88o), une enquête gouvernementale fait apparaître que les montagnes à fourrage constituaient une part importante des I 200 ha des forêts villageoises de l'époque, notamment dans les villages vivant des montagnes (Wakasugi, Sasaguri, Haginoo) (voir le tableau ci-dessous) ${ }^{78}$. Ces chiffres montrent aussi que plusieurs villages étaient dénués de telles ressources (Tanaka sans montagne du tout, Oto.inu, Onaka et Wada sans sources fourragère).

\begin{tabular}{|c|c|c|c|c|c|c|c|}
\hline 1880 & 总 & 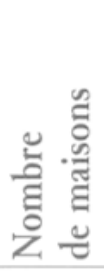 & 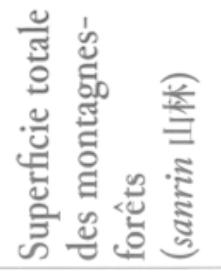 & 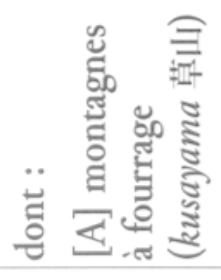 & 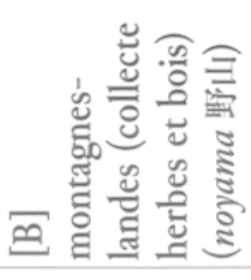 & 希 & : \\
\hline Oto.inu & 329 & 70 & 2 chō, 3 tan & o chō, 9 tan & o & 32 & II \\
\hline Wakasugi* & 324 & 64 & 126,6 & $45 c h o ̄$ & o & 53 & 7 \\
\hline Onaka & 485 & 106 & 5,6 & ० & O chō, I5 tan & 43 & 33 \\
\hline Sasaguri & 1342 & 265 & 704 chō & 228,9 & $2 c h \bar{~}$ & 75 & 53 \\
\hline Kana.ide & 187 & 36 & 31,7 & 6,3 & o & 23 & 6 \\
\hline Haginoo & 210 & 42 & 117,5 & IOO $c h o ̄$ & o & 44 & 4 \\
\hline Tanaka & 152 & 30 & o & o & o & IS & 9 \\
\hline Takata & 227 & 47 & 33,2 & $5 \mathrm{cho}$ & ० & I3 & $2 \mathrm{I}$ \\
\hline Tsubakuro & 206 & 45 & I4I, 6 & 7 chō & o & I4 & I4 \\
\hline Wada & 340 & 74 & 31,9 & ० & o & 15 & 28 \\
\hline
\end{tabular}

'En 1954, on compte 45 bovins mais aucun cheval dans les 45 maisons de Wakasugi ${ }^{79}$.

78. Nishi Nihon Bunka kyōkai, éd., Fukuoka-ken chiri zenshi I, vol. 22, p. 458-501. Je reprends

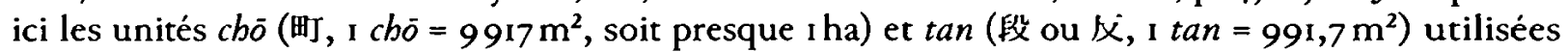
dans le document, en arrondissant aux tan et supprimant les deux plus petites unités se 腩 et $b u$ 步. $C h o ̄$ et hectares étant à peu près équivalents, chō sera utilisé aussi ci-après. Les superficies ainsi calculćcs sont inférieures à celles des forêts actuelles, or les surfaces forestières n'ont pas augmenté depuis Meiji. Aussi, selon les responsables de la mairie de Sasaguri, ces chiffres sont-ils à prendre comme une estimation des proportions plutôt que comme des valeurs exactes.

79. Gōya Takeshiro, Chikuzen Wakasugi kyödoshi, p. 124 . 
C'est dans ces espaces également que l'on prend les graminées (kaya 菅 Poaceae, notamment chigaya 茅 Imperata cylindrica et susuki 薄, le miscantbus) pour recouvrir les toits une fois tous les dix ans. Car jusqu'à Meiji, seuls les toits de chaume ou de planches étaient autorisés pour les habitations paysannes. Toutes ces pratiques concouraient ainsi à la déforestation non seulement des pentes proches des villages qui étaient alors sans arbres, mais aussi de pans entiers de la « montagne des terres reculées ». Jusque dans les années 1920, les monts Takatorii 高鳥居山, Kome, Hokotate, Yamate et la partie nord de Takata sont recouverts de miscanthus. Ceci d'autant plus que, jusque dans la première moitié du $\mathrm{xx}^{\mathrm{e}}$ siècle, les brûlis sont pratiqués sur ces mêmes pentes pour laisser l'espace libre aux herbes à fourrage, ou avant de semer le sarrasin (soba 菜 Fagopyrum esculentum), le millet, le colza, ou d'y planter les tubercules. Début Meiji, ces champs sur brûlis représentaient encore 70 ha sur les 500 ha de cultures ${ }^{80}$.

Situées un peu plus haut, les forêts de feuillus sont elles aussi constamment exploitées pour le combustible : le bois (maki 薪) pour les fourneaux domestiques, et le charbon de bois (sumi 炭, mokutan 木炭) pour les braseros. Fabriqué principalement à partir des chênes (kunugi, Quercus acutissima), le charbon de bois représentait un apport financier non négligeable car il était en partie vendu aux habitants de la partie urbanisée de Sasaguri et dans les villages manquant de forêts. En I880, le village de Sasaguri en déclarait 35000 kin (21 tonnes) ${ }^{81}$, Kana.ide 900 sacs (soit environ 9 tonnes). En 1907, les quatre villages composant Sasaguri déclaraient en produire annuellement une totalité de 4500 sacs (soit environ 45 tonnes) ${ }^{82}$. Jusque dans les années 1950, durant les mois d'hiver, les habitants construisaient des fours à charbon de bois (kama 䉓) dans leurs propres forêts de feuillus, en bordure des chemins pour faciliter le transport, alors que les professionnels du charbon de bois les édifiaient au fond des montagnes. Lorsqu'il n'y avait plus assez de bois sur place, certains partaient en acheter dans d'autres forêts (celles des autres) hors du village et même de la commune, où ils faisaient sur place le charbon de bois qu'ils vendaient, notamment aux citadins de Fukuoka. Tout ceci pose une double question à laquelle il sera répondu plus loin : pourquoi un si petit territoire communal n'a-t-il pas été entièrement dévasté de longue date par une telle surexploitation, ne laissant que des «montagnes chauves » ${ }^{83}$, et que faisait-on dans les villages où il n'y avait pas de montagne-forêt ?

Il faut noter également les coupes régulières faites pour le bois de charpente des habitations locales. Cette demande va exploser avec ce qui a été appelé le «boom des constructions en bois ", dans la période de l'après-guerre. En effet, la brusque montée des besoins massifs en bois pour la reconstruction des habitations détruites dans tout le pays durant la guerre, et à l'instar de ce qui s'est passé partout sur le

80. Sasaguri-chō bunkazai senmon-iinkai, éd., Sasaguri chōshi. Minzoku-ben, p. 8I.

81. $\mathrm{I}$ kin $斤=0,600 \mathrm{~kg}$.

82. Sasaguri-chō bunkazai senmon-iinkai, éd., Sasaguri chōshi. Minzoku-ben, p. 28. Ces chiffres correspondent aux denrées imposables.

83. Chiba Tokuji 下葉徳爾, Hage yama no bunka はげl」の文化, coll. «Nihon no rekishi chiri » U本の糜史地理 4 , 1973, vol. 4 . 
territoire national, on assiste à un spectaculaire développement des plantations et de l'exploitation forestière à Sasaguri. Ceci coïncide avec une période de grande prospérité pour les habitants.

Enfin, d'une façon générale, pour les villages des secteurs montagneux, les produits de l'agriculture (petite riziculture et surtout polyculture de blé et légumes) et ceux de la montagne (bois et produits des collectes : pousses de bambou, châtaignes, fruits et plantes sauvages saisonnières, champignons, herbes médicinales) étaient utilisés non seulement pour l'autoconsommation mais aussi pour la vente. Hommes et femmes pratiquaient la vente ambulante avec une carriole à bras, dans la commune, mais aussi jusqu'à Fukuoka, notamment pour le charbon de bois. Ainsi, toutes les femmes d'Oto.inu pratiquaient cette vente, notamment jusqu'en I965 auprès des mineurs de la commune. Cette activité marchande a permis à de petits groupes de familles d'habiter dans les zones montagneuses isolées. Ainsi, dans les années 1920-1930, quelques maisons installées près de l'étang Ōkura 大倉池 dans la montagne de Kana.ide subvenaient à leurs besoins en consommant et vendant exclusivement les produits de la montagne, avant qu'elles ne descendent s'installer en bas dans la zone des habitations, après la guerre. Il ne faut pas non plus oublier que les ustensiles domestiques et les instruments de travail étaient majoritairement en bois et en bambou jusqu'à une date récente. Les bambous coupés dans la montagne du village ${ }^{84}$, pour l'usage local et pour la vente, avaient de multiples utilisations : renforcement des diguettes et barrages de l'eau des rizières, perches pour le séchage du riz moissonné et aussi celui du linge, etc.

On comprend donc que, pour les habitants de Sasaguri, l'une des conséquences de la continuité de toutes ces pratiques sur ce territoire communal exigu soit la connaissance exacte et extrêmement détaillée des lieux, de la flore et de la faune, des rythmes saisonniers, dc l'histoirc de ces milieux, des techniques et instruments, c'est-à-dire de tout ce qui concerne leur environnement, connaissance détenue par tous ceux qui ont ou avaient à travailler dans les montagnes-forêts. Certains d'entre eux sont intarissables et toujours prêts à servir de guides jusqu'aux lieux les plus reculés dont les moindres détails n'ont aucun secret pour eux. Cette connaissance ancrée dans une expérience coutumière constitue un patrimoine cognitif local - discursif, comportemental, pratique - qui s'est transmis jusqu'à maintenant parce qu'il était ancré dans les usages. Mais il semble se trouver aujourd'hui mis en danger par l'absence de relais de transmission dans le registre des pratiques de subsistance. Pourtant, comme nous le verrons, la transformation du regard vers la montagneforêt et celle de ses utilisations les plus récentes pourraient provoquer un nouveau tournant. L'histoire montre en effet que ce patrimoine a récemment déjà traversé plusieurs fois des changements locaux et globaux assez considérables.

Pour caractériser les bouleversements induits à l'époque contemporaine par l'impact des conditions économiques et politiques extérieures sur les communautés locales, certains ethnologues opposent l'héritage de la «culture du bois » ( $k i$ no bunka 木の文化) à l'avènement moderne de la «culture du fer » (ou du métal) (tetsu

84. Dicton de Wakasugi : «Les arbres, coupe-les à six (c.-à-d. en juin), les bambous à huit

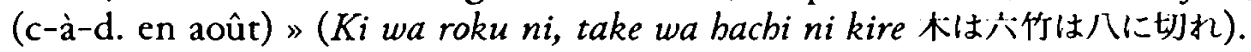


no bunka 鉄の文化) $)^{85}$. Deux facteurs principaux liés à l'extérieur des villages ont en effet bouleversé cette économie micro-locale fondée sur le bois. Ce sont, au début du $\mathrm{Xx}^{e}$ siècle, l'introduction des instruments en métal et les trois crises du bois et du combustible. La première est l'aspect visible de la transformation économique initiée à Meiji. Reposant sur un système d'échanges à une échelle régionale et nationale, et sur la circulation des personnes, des produits, des espèces, la commercialisation des instruments en métal a imposé la nécessité d'acquérir des espèces en sortant des villages pour former ou intégrer des circuits marchands extérieurs. L'expression " après la guerre russo-japonaise » (1904-1905) désigne le ressenti de cette onde de choc dans les localités agricoles, qui se " modernisent ", non pas dès le début de Meiji, mais lentement à partir des années $1910^{86}$. Utilisée à la fin des années 1960 par les paysans âgés qui avaient vécu cette époque, cette expression dénote à la fois l'importance du temps dans les bouleversements socio-environnementaux et le côté drastique des changements provoqués. Selon les localités et les registres de pratiques, il a fallu entre quarante et quatre-vingt ans pour que l'impact de Meiji provoque des modifications déterminantes et durables dans les systèmes locaux. Les secondes, les trois crises du bois et du combustible, constituent l'arrière-plan national et mondial de ces transformations locales ${ }^{87}$.

Il s'agit de crises soit de surexploitation des forêts, soit de leur abandon, qui reposent sur la pénurie ou l'excédent, et le changement de la valeur du bois. La première crise fut la dévastation des forêts engendrée par les coupes sauvages qui suivirent le remaniement du régime des propriétés à Meiji, à la suite de quoi fut élaborée une législation sévère qui permit la reconstitution des forêts villageoises. La seconde surexploitation des forêts à l'origine des « montagnes chauves » date de la guerre du Pacifique et de l'appauvrissement de toute la population. Cette crise fut surmontée, d'une part, par les campagnes de plantations forestières des années I950 pour la reconstruction du pays - mais celles-ci ayant une visée économique de rendement, elles provoquèrent une autre forme de surexploitation, industrielle, et l'invasion de mono-peuplements de conifères ; et, d'autre part, par la révolution du combustible dans les années 1955-1975, au cours desquelles le charbon de bois et le bois furent supplantés par le pétrole et le gaz pour le chauffage et les besoins énergétiques (ce à quoi on peut ajouter aussi le remplacement des engrais naturels par les engrais industriels). La troisième crise remonte aux années 1970-1980, avec l'ouverture du marché du bois étranger qui provoqua la chute des prix et la mévente des bois nationaux. Ceci intensifia la migration vers les villes, et la diminution ou la disparition des populations locales travaillant dans les montagnes. Ces deux dernières crises contribuèrent à accélérer, surtout à partir des années 1990, l'abandon de l'entretien des forêts de feuillus et de conifères, engendrant ainsi de graves dégâts environnementaux. Les enquêtes gouvernementales montrent qu'en $2006,80 \%$ des forêts japonaises ne sont pas entretenues. Et alors que les deux tiers du pays sont

85. Voir les analyses de Sasaki Tetsuya 佳々林折哉 sur l'ethnographie qu'il a conduite dans le département de Fukuoka. No no kioku. Hito to kurashi no genzōo野の嗼憶——人と無らしの原像, 2007.

86. Sasaki Tetsuya, op. cit., p. 174 et sq.

87. Arioka Toshiyuki, Sato-yama II. 
constitués d'espaces forestiers, le Japon est l'un des plus gros importateurs de bois du monde ( $80 \%$ du bois utilisé est importé) ${ }^{88}$. Mais, comme l'indique l'émergence de la question du sato-yama, depuis les années 2009-2oro s'effectue à nouveau une réévaluation de l'importance du capital forestier tant en termes de ressources matérielles que sociales et culturelles. C'est donc dans ce contexte extérieur et intérieur caractérisé par les retournements contemporains extrêmes, que va se jouer l'avenir du patrimoine de connaissances environnementales des populations villageoises.

Espace végétal aux cycles maîtrisés pour les besoins locaux à la fin du XIX ${ }^{e}$ siècle, les montagnes ont vu leur aspect se transformer au siècle suivant sous l'effet des dévastations répétées dénudant leurs pentes, puis suite à une mono-exploitation intensive ou à leur abandon aux repousses sauvages. Ces modifications accélérées du paysage local sont l'expression du changement des usages et des modes de vie, c'est-à-dire aussi des règlementations et des normes qui ont constamment formaté ces usages. Ceci pose la question de la façon dont le système social local a résolu les problèmes de gestion de ces ressources au cours de l'histoire.

\section{Structures et législation : les régimes de propriété dans l'engrenage des réformes nationales}

Ayant comme arrière-plan les transformations considérables des modalités locales du recours aux arbres et au bois, la diversité qui caractérise les zones forestières de Sasaguri est en effet aussi la résultante de l'intrication historique, sur ces espaces, de trois systèmes complexes et respectivement multi-strates. Ces systèmes dépendent à la fois de l'intérieur et de l'extérieur de la commune. Ce sont les régimes de propriété, les dispositifs de gestion et les systèmes d'exploitation et d'usage. Les changements drastiques qui ont affecté la propriété foncière à Meiji, puis au cours du $\mathrm{xx}^{\mathrm{c}}$ siècle, ont eu un impact déterminant sur les mutations des systèmes de gestion et sur les usages des espaces forestiers. En outre, propriété, gestion et exploitation d'un même espace peuvent être aux mains d'organisations distinctes. Avant d'aborder quelques types de modalités concrètes d'articulation de ces systèmes à Sasaguri, j'examinerai la question foncière sous l'angle de l'impact local des dynamiques externes à la commune.

Aujourd'hui ${ }^{89}$, les 2395 hectares de bois de Sasaguri sont répartis entre deux catégories de propriétaires : les propriétaires privés (I $989 \mathrm{ha}$ ) et le ministère des Forêts, c'est-à-dire l'État (406 ha) ${ }^{90}$ (carte 4$)$. Les forêts domaniales (kokuyū-rin 国有林, seulement $17 \%$ de la totalité forestière), constituées entièrement de conifères, sont situées d'une part, au sud, sur la partie nord-ouest du mont Wakasugi et d'autre part, au nord, dans les parties montagneuses les plus hautes au nord-est de Yamate et de Kido, c'est-à-dire, dans les deux cas, dans les endroits les plus éloignés des zones habitées. Toutes les autres sont des forêts privées (min.yū-rin 民有林, littéralement

88. Shinrin shigen no genkyō genjō : voir ci-dessus, n. 67.

89. Chiffres communiqués par la mairie de Sasaguri, en février 2012.

90. Tous les chiffres ci-joints proviennent du recensement national de 2009. Voir la répartition sur la carte 4 des différentes propriétés forestières. 
《du peuple $\left.{ }^{91}\right)$, largement majoritaires à Sasaguri $\left(83 \%\right.$ de l'ensemble ${ }^{92}$, et sont partagées entre les propriétés des particuliers (shiyū-rin 私有林, $\mathrm{I} 376 \mathrm{ha}$ ), les propriétés des collectivités territoriales, dites " publiques 》 (kōyü-rin 公有林) : départementales (Fukuoka, 132 ha), communales et de collectivité de communes (283 ha), et les propriétés collectives (kyōyu-rin 共有林, ici, les secteurs patrimoniaux ${ }^{93}$ zaisanku 財産区 $198 \mathrm{ha}$ ). Au nombre des propriétés privées sont comptées celles des temples et des sanctuaires, et celles de l'Institut de recherche sur les forêts de l'université de Kyūshū (depuis la législation de 2004 sur l'autonomie des universités).

L'évolution du système foncier depuis l'époque d'Edo jusqu'à nos jours s'est faite sous la forme d'un renversement paradoxal. En effet, non seulement une partie des propriétaires actuels vit hors de la commune, mais la majorité de ceux qui y résident ne sont pas des usagers des forêts, ne pratiquent pas eux-mêmes l'exploitation, ou l'ont même abandonnée, et n'en vivent pas. À l'inverse, à l'époque d'Edo et jusqu'en I871, c'est l'absence de propriété privée (de type contemporain) qui caractérise le système foncier. Les habitants, s'ils en avaient l'usage, ne détenaient pas de propriétés forestières privées, alors même que les forêts étaient pour eux des ressources vitales. Quant aux terres agricoles, en dehors de quelques gros propriétaires terriens, c'est l'exiguïté voire l'absence de propriété qui était la norme pour la plupart des paysans ${ }^{94}$. Mais tous résidaient sur les terres dont ils vivaient et pour lesquelles ils payaient au fief ce qui légitimait leur statut social : des redevances en riz sous la forme d'un impôt villageois (en plus de celui qu'ils versaient au propriétaire s'ils étaient fermiers) et de services communautaires (creusement et nettoyage des étangs d'irrigation, entretien des ponts et des routes). Ils jouissaient en outre d'un droit d'usage sur les montagnes à fourrage, en échange de devoirs communautaires (surveillance, entretien

91. La distinction juridique de toutes les forêts japonaises en deux catégories domaniales et privées date de la loi sur les forêts de i95I (loi actuelle : I, 2, [3]).

92. Ce pourcentage est supérieur à la moyenne nationale : les forêts privées (17410000ha) représentent $69,36 \%$ du total des forêts japonaises (25100000ha), et les 7690000 ha de forêts domaniales 30,63\% (recensement du Rin.yachō 2007).

93. Système créé en 1888 au moment des premières fusions communales, et perpétué par les législations ultérieures, accordant aux anciens villages la possibilité d'obtenir le statut de propriété privée pour les terres gérées collectivement jusque-là. Il a pour particularité d'imposer un retour des bénéfices tirés de ces secteurs à la collectivité qui les gère, dans la continuité d'une conception et des usages des anciens communaux. C'est l'un des moyens utilisés pour garder les anciens communaux. En 2010, il y avait une totalité de 300000 ha de forêts et de landes en secteurs patrimoniaux sur le territoire national (site du ministère de l'Intérieur, Sōmushō 総移省, document intitulé 《 7-22 todōfuken, shoyū keitai-betsu genkyō sbinrin menseki » 都道府県, 所有形 柋别現況森林面筫.

94. Dans la première moitié du XIX siècle, les propriétaires de plus de 3 chō (environ 3 ha) de rizières et de champs ne représentaient que 1,6 et $2 \%$ de la population des plus gros villages agricoles de Takata et Onaka. Il n'y en avait aucun à Tanaka. En moyenne, ceux qui étaient propriétaires possédaient entre 1 et $4 \tan \left(1000\right.$ à $\left.4000 \mathrm{~m}^{2}\right)$ cultivables. Mais d'autres, sans aucune terre, n'étaient que paysans-domestiques (nago 多子) chez les gros propriétaires ( 7 à Onaka) (Sasaguri chōshi, p. 121-122). Dćbut Meiji, en $1880,5000 \mathrm{~m}^{2}$ était la moyenne des propriétés (rizières et champs secs) dans les dix villages composant l'actuel Sasaguri (Nishi Nihon Bunka kyōkai (éd.), Fukuoka-ken chiri zenshi, vol. 6, cité dans Sasaguri chöshi, p. 219). 


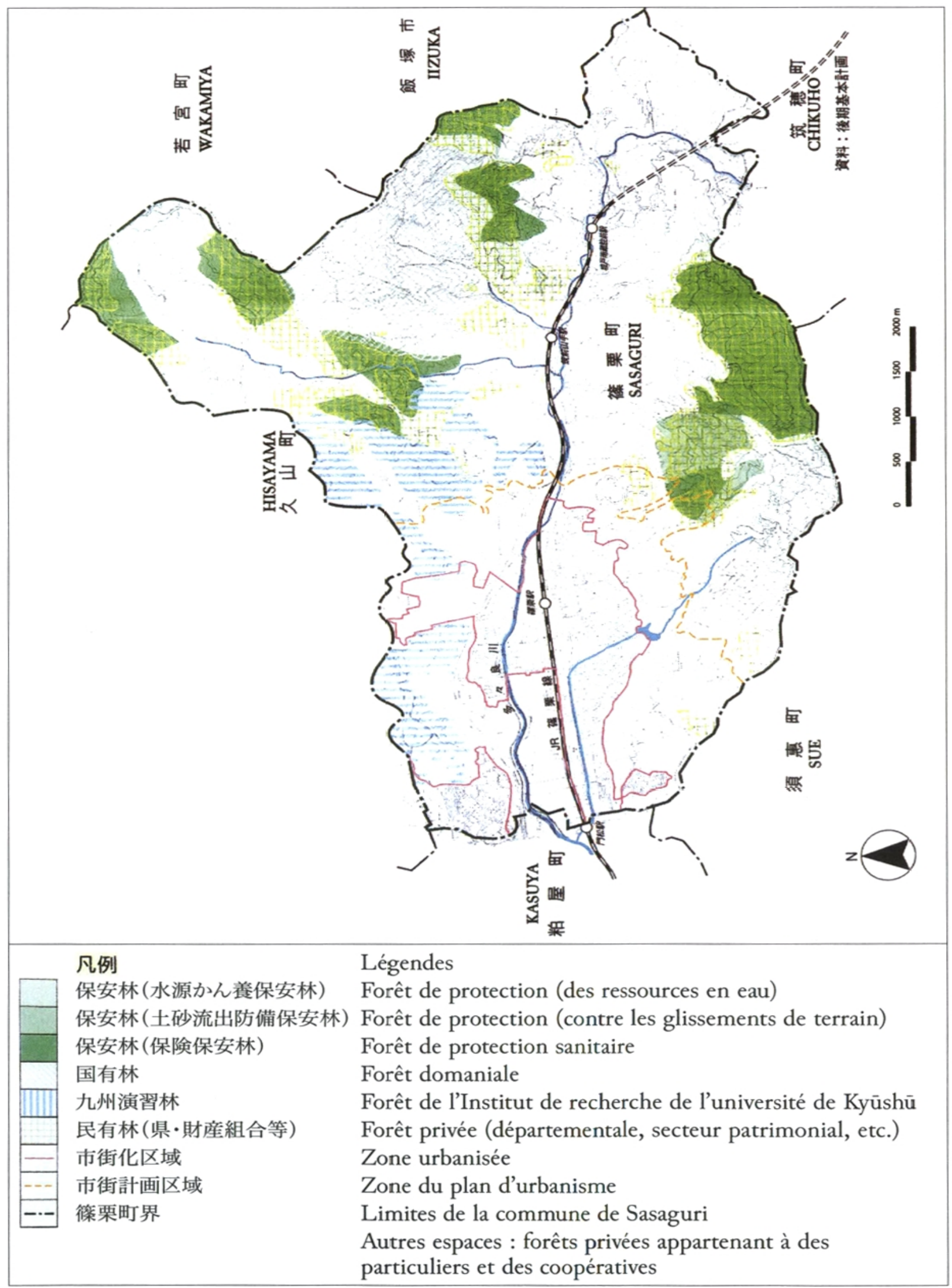

Carte 4 : Les différents types de forêts et de propriétés forestières. (Sasagurichō kikaku kōhōka 箫栗町企画広報課, 2003. Green Oasis Sasaguri. 2003 Dai yoji Sasaguri-cbō kokudo riyō keikaku 第四次籍栗町国土利用計画, Sasaguri : carte 5.) 
des forêts, services lors des chasses du daimyō, etc.) qui garantissaient ces droits. Ne pas remplir ces devoirs était pénalisé par le retrait des droits communautaires.

Ce système généralisé à l'époque d'Edo ne fut que formellement transformé à Meiji avec l'abolition du système féodal, puisque les chefs des anciens fiefs demeurèrent de fait de grands propriétaires rentiers qui, moyennant des redevances en nature, louaient leurs terres aux exploitants fermiers. Il fallut attendre la réforme agraire et l'instauration de la propriété privée réelle de l'après-guerre pour que le système foncier soit véritablement modifiép5.

À Sasaguri, comme dans tous les villages du fief de Fukuoka (dit fief des Kuroda, du nom de la famille des daimyō détenteurs de l'autorité de 1600 à 1871), durant toute l'époque d'Edo les terres de montagne (dites o-[ou mi-]yama 御山, la Montagne) étaient la propriété du fief. Leurs forêts étaient source de revenus importants et faisaient l'objet d'une administration sourcilleuse, d'une surveillance constante et furent parfois surexploitées pour les besoins des daimyō successifs.

Or à Meiji, suite à l'abolition des fiefs, l'État confisque ces anciennes terres féodales, notamment celles des montagnes-forêts. Avec les terres enlevées aux temples et aux sanctuaires, dans un premier temps, celles-ci viennent constituer les forêts " gouvernementales 》 (kan-rin 官林) qui deviendront ensuite les forêts domaniales (kokuyü-rin 国有林 $)^{96}$. En I893, leur superficie totale va jusqu'à s'élever à 19 I8000o ha, soit $64 \%$ de la totalité des forêts japonaises de l'époque ${ }^{97}$. Une partie néanmoins put être conservée par les communautés locales. Ce fut le cas des montagnes qui étaient la propriété des villages, et celles pour lesquelles existe un autre type de droit de propriété. Ce droit concerne tout ce qui pousse sur le sol et peut être détenu par un propriétaire différent du tréfoncier. Il s'agit du kejō-ken 毛上権 «droit sur le végétal du dessus » ${ }^{98}$, ou cbijo-ken 地上権 «droit de superficie », que je traduis par droit (de propriété) arboraire, terme utilisé pour désigner ce même droit ancien dans d'autres sociétés, notamment du pourtour de la Méditerranées. Ancrée dans le droit coutumier ancien, cette particularité est

95. Pour une présentation en français de l'évolution moderne du système foncier, voir Marc Bourdier \& Philippe Pelletier (éd.), L'archipel accaparé. La question foncière au Japon, 2000.

96. Les mesures prises par le gouvernement de Meiji pour règlementer et gérer l'espace forestier du pays sont loin d'avoir été cohérentes au début. Les objectifs mal définis et les excès de la législation ont suscité des révoltes parmi les populations paysannes, des procès engagés par les temples et sanctuaires lésés, ce qui a contraint les législateurs à modifier de nombreuses fois

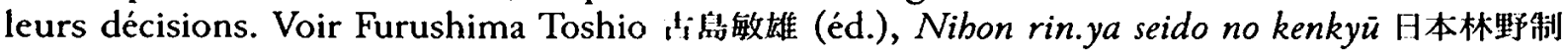
度の研究, 1955 .

97. Soit plus de 2 fois er demi de leur superficie actuelle. Okuchi Shō 奥地正, "Nihon kokuyūrin ni okeru rōdō soshiki no keisei to tenkai »国有林における多働組織の形成と展開 I, Ritsumeiknan keizaigaku 立命飭経济学 23, vol. 4 (1974), p. 447-489.

98. Kejo-ken 毛上権, littéralement « droit sur ce qui se dresse/pousse sur le dessus ». Code civil japonais, article 265 et $s q$. En France, ce droit, qui existait en droit romain, n'est pas réglementé par le Code civil mais par la jurisprudence (art 552 du Code civil) et la Loi du io janvier 1824 concernant le droit de superficie et d'emphytéose.

99. Florence Jean, La propriété arboraire en Corse et dans les pays environnants d'Europe et du pourtour de la Méditerranée, 200I. Diego Moreno, Lada Hordynsky-Caillat, Odile Redon, Silvano Serventi, "Châtaigneraie "historique" et châtaigneraie "traditionnelle". Notes pour l'identification d'une pratique culturale » Médiévales 16, vol. 17 (1989). Ces travaux montrent les similarités entre 
aujourd'hui fixée par la législation japonaise, pour laquelle non seulement le bâti, mais aussi tout ce qui se trouve à la surface du sol fait l'objet d'un droit de propriété distinct de celui du terrain. En outre, le législateur de Meiji reconnut aux communautés locales un droit de communaux ${ }^{100}$. Ces divers droits furent déterminants à Meiji, puisque l'État renonça à accaparer les terres féodales où ils s'exerçaient, et accepta de les attribuer ou de les brader aux collectivités ou individuellement aux paysans, pourvu que leur usage constant dans les périodes antérieures ait pu être prouvé par des documents. Ainsi les habitants de Sasaguri purent-ils acquérir un certain nombre de ces terres en faisant valoir leurs droits d'usage sur celles-ci, d'une part, en tant que communaux (forêts, pâturages pour le fourrage et autres productions végétales) dits iri.ai yama 入会山 (《montagne où l'on entre ensemble ») qui constituaient les ressources indispensables aux communautés villageoises, ou, d'autre part, sur des terres dont les droits d'exploitation forestière leur avaient été remis par le fief, ou par donation.

Les registres fonciers ${ }^{101}$ sont les témoins de ces bouleversements des structures locales contraintes par les changements du régime foncier étatique et la modification de la législation. Mais les critères des relevés ne sont pas toujours clairs ni unifiés. Néanmoins, si l'on reprend le relevé de 1880 , on constate que chaque village a pu conserver une partie des anciennes montagnes-forêts du fief. Il s'agit de montagnes pour lesquelles existaient des documents prouvant que les villageois, paysans et petits guerriers locaux, jouissaient de longue date d'un droit de propriété arboraire. Elles se répartissent en sept catégories. Outre les forêts gouvernementales, cellesci comptaient : les « anciennes montagnes à attestation 》 (moto shömon yama 元証. 文山 - no 3 du tableau p. I63 - avec droit exclusif et nominatif sur demande, moyennant travail de plantation et d'entretien ; système initié au XVII siècle pour replanter les montagnes chauves) ; les « anciennes montagnes confiées à quelqu'un » (moto azukari yarna 元預山 - $\mathrm{n}^{\circ} 4$, confiées sur demande locale adressée au fief, avec droit d'usage pour collectes d'herbes et de bois pour le feu, en échange de l'entretien, avec vérification tous les Io ans par le fief) ; les " anciennes montagnes-tenures » (moto bairyō yama 元搝領山 - $\mathbf{n}^{\circ} 5$, donations à des guerriers) ; les montagnes des sanctuaires (shayama 社山— $\mathrm{n}^{\circ}$ 6) ; les montagnes-landes (noyama 野山 - $\mathrm{n}^{\circ} 7$; avec droit d'usage pour collectes d'herbes et de bois pour le feu) dont les fonctions étaient les mêmes que celles des « montagnes à fourrage » (kusayama 草山 - $\left.\mathrm{n}^{\circ} 2\right)^{102}$.

On remarque l'importance des montagnes à fourrage ( 2 et $7: 32,9 \%$ ) par rapport aux autres $(3,4,5,6)$ dont la totalité ne représente que $7,1 \%$, et celle de l'espace

\footnotetext{
le cas japonais et les très complexes régimes coutumiers médiévaux méditerranéens, notamment pour les châtaigneraies.

IOO. Iri.ai-ken 入会権 date de 1889. Code civil, 263 et 294.

IOI. Succédant en 1947 aux relevés cadastraux (jiyose-chō 地寄帳, « registre des relevés fonciers») (complété par le "registre nominatif [des propriétés] ", nayose-chō 名奇帳), le registre foncier (tochi daichō 土地台帳) subsista jusqu'au changement de législation en 1960, date à partir de laquelle il est remplacé par le registre public des actes civils (tōki-bó 登記簿). Existait également un registre des montagnes, yamane-chō 山作帳.

I02. Sur l'historique de ces appellations dans le fief de Fukuoka, voir Kondō Tenji 近藤

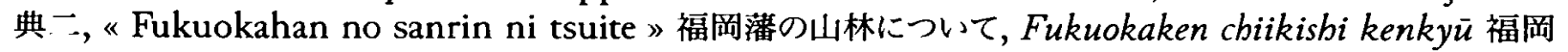
県地域史研究 I2 (1993).
} 
accaparé par l'État (i : 60,7\% des montagnes-forêts). Ces montagnes à fourrage étaient des communaux.

Mais toutes les terres ne purent être récupérées par les habitants. Aussi, comme nous le verrons dans le cas de Wakasugi, à Sasaguri, comme dans de nombreuses autres localités du pays, s'engagèrent alors de très longs procès des communautés locales contre l'État pour en revendiquer la restitution. Peu d'entre elles eurent gain de cause.

Avec le transfert de la propriété aux paysans ${ }^{103}$, l'objectif du nouveau gouvernement était de rompre avec le système Tokugawa qui contrôlait directement les terres. Ayant pour but la construction de l'État-nation, il voulait, par la création d'impôts fonciers en espèces et non plus en nature, établir des finances nationales en mettant à leur fondement la totalité des revenus et des biens. Or, par les modalités mêmes des rapports qu'ils entretenaient avec les montagnes-forêts, les villageois étaient attachés principalement à ce qui poussait sur leurs pentes, beaucoup plus qu'à la propriété des montagnes elles-mêmes. Aussi s'estimèrent-ils lésés d'être obligés de payer pour utiliser des espaces sur lesquels les seuls droits d'usage qu'ils possédaient en commun jusque-là leur suffisaient. Ils cherchèrent donc à conserver sous la forme de propriétés communautaires ces montagnes dont les ressources leur étaient indispensables. Mais c'est précisément ce que les gouvernements successifs, d'abord à Meiji puis au $\mathrm{xx}^{\mathrm{e}}$ siècle, vont s'appliquer à démanteler. Car ce fonctionnement communautaire coutumier a été considéré comme l'un des piliers des structures et solidarités locales qu'il fallait faire éclater pour réorganiser la population sous une autorité centralisée. Dans ce but, tout au long du $\mathrm{xX}^{e}$ siècle, a été menée une politique drastique de restructuration foncière inscrite dans le processus continu de remembrement des communes. La méthode utilisée a été de faire fusionner les anciens villages pour les regrouper en des entités administratives de plus en plus grandes, et, à l'inverse, de multiplier les propriétés privées.

Ce processus s'est déroulé en trois étapes majeures dites des « trois grandes fusions de Meiji, Shōwa (double étape de centralisation) et Heisei (décentralisation) »104. C'est dans ce cadre que, par la réunion en 1889 de six et quatre des anciens villages, sont d'abord formées respectivement les deux unités administratives villageoises de Seto et Sasaguri ; puis, que, en 1955, celles-ci ont été réunies en une seule unité communale prenant le nom de Sasaguri. Mais en 2010, après plusieurs années de concertation avec sept communes et une ville voisines, Sasaguri a renoncé à engager le dernier en date des processus de grande fusion. Lancée par l'État en 2000 , cette fusion de Heisei a pour but de renforcer la décentralisation et l'autonomie des

103. Ordonnance du $18^{e}$ jour du $12^{e}$ mois de 1868 (Meiji I), Dajōkan fukoku 太政官布告.

IO4. I) Loi de I889 (Meiji 22) : les 71314 entités villageoises et urbaines de l'époque d'Edo sont réduites au nombre de 15859 villes, communes et villages. 2) Loi de 1953 (Shōwa 28) : de 9868 , les unités territoriales passent à 3472 (1961). 3) Réforme des administrations locales et décentralisation à partir de 2000 (Heisei 12) : le nombre actuel (janvier 2013) de villes, communes et villages ne s'élève plus qu'à $\mathbf{7} 719$. 


\begin{tabular}{|c|c|c|c|c|c|c|c|c|c|c|c|c|c|c|c|c|}
\hline \multirow{2}{*}{$\begin{array}{r}\text { I880 } \\
\text { Oto.inu }\end{array}$} & \multicolumn{2}{|c|}{$\begin{array}{c}\text { Superficie totale } \\
\text { des montagnes- } \\
\text { forêts } \\
\text { (sanrin } \text { 山林) }\end{array}$} & \multicolumn{2}{|c|}{$\begin{array}{c}\text { I. } \\
\text { forêt } \\
\text { gouvernementale } \\
\text { (kan-rin 官林) }\end{array}$} & \multicolumn{2}{|c|}{$\begin{array}{c}2 . \\
\text { montagne à } \\
\text { fourrage } \\
\text { (kusayama 草山) }\end{array}$} & \multicolumn{2}{|c|}{$\begin{array}{c}3 . \\
\text { ancienne } \\
\text { montagne à } \\
\text { attestation } \\
\text { (moto shömon } \\
\text { yama 元詿文山) }\end{array}$} & \multicolumn{2}{|c|}{$\begin{array}{c}4 . \\
\text { ancienne } \\
\text { montagne } \\
\text { confiée à qqn } \\
\text { (moto azukari } \\
\text { yama 元預山) }\end{array}$} & \multicolumn{2}{|c|}{$\begin{array}{c}5 . \\
\text { ancienne } \\
\text { montagne- } \\
\text { tenure } \\
\text { (moto bairyō } \\
\text { yama 元扯領山) }\end{array}$} & \multicolumn{2}{|c|}{$\begin{array}{c}6 . \\
\text { montagne } \\
\text { du sanctuaire } \\
\text { (shayama } \text { 社山) }\end{array}$} & \multicolumn{2}{|c|}{$\begin{array}{c}7 . \\
\text { montagne-lande } \\
\text { (noyama 野山) }\end{array}$} \\
\hline & $2 c h \bar{o}$ & $3 \tan$ & ochō, & $2 \tan$ & ochō, & 9 tan & ochō, & I4tan & ochō, & stan & & o & ochö, & Itan & & $\mathrm{O}$ \\
\hline Wakasugi & I26 chō, & $6 \tan$ & 79 chō, & $2 \tan$ & $45 \mathrm{cho}$ & & & 0 & I cho, & Itan & & 0 & I $c h \bar{o}$ & $3 \tan$ & & 0 \\
\hline Onaka & schō, & $6 \tan$ & Is chō & & & o & & $4,8 \tan$ & ochō, & Itan & & 0 & ochō, o, & ,or $8 \tan$ & ochō, & Istan \\
\hline Sasaguri & 704 chō & & $427 \mathrm{cho}$ & $3 \tan$ & $228 \mathrm{cho}$ & $9 \tan$ & & $9,8 \tan$ & & o & $35 \mathrm{cho}$, & $2 \tan$ & I chō, & $3 \tan$ & $2 c h o ̄$ & \\
\hline Kana.ide & 3I $c h \bar{o}$ & $7 \tan$ & I6 chō, & stan & 6 chō, & $3 \tan$ & & 0 & $3 \mathrm{cho}$ & $9^{\tan }$ & $4 c h \bar{o}$ & & ochō, & $2 \tan$ & & $\mathrm{O}$ \\
\hline Haginoo & II7chō, & stan & I7 chō, & $4 \tan$ & Ioochō & & & 0 & & 0 & & o & ochō, & Itan & & $\mathrm{O}$ \\
\hline Tanaka & & o & & 0 & & o & & 0 & & $\mathrm{O}$ & & o & & 0 & & $\mathrm{O}$ \\
\hline Takata & $33 c h o \overline{,}$ & $2 \tan$ & I9 chō, & $8 \tan$ & schō & & & 0 & ochō, o & $0,05 \tan$ & 8 chō, & $3 \tan$ & & 0 & & O \\
\hline Tsubakuro & I4I $c h o \bar{o}$ & $6 \tan$ & I34chō, & $3 \tan$ & $7 c b o ̄$ & & & 0 & & o & & 0 & & 0 & & o \\
\hline Wada & 3I $c h \bar{o}$ & $9 \tan$ & 3I $c h \bar{o}$, & Itan & & 0 & ochō, & $4 \tan$ & & $\mathrm{O}$ & & $0,4 \tan$ & & 0 & & o \\
\hline$\%$ & & $100 \%$ & & $60,7 \%$ & & $32,8 \%$ & & $2,4 \%$ & & $0,4 \%$ & & $4 \%$ & & $0,3 \%$ & & O,I $\%$ \\
\hline
\end{tabular}

Nishi Nihon Bunka kyōkai (éd.), Fukuoka-ken cbiri zenshi, vol. 22 (cité dans Sasaguri cböshi, p. 219 ; les chiffres des petites unités ont été arrondis). 
collectivités locales en faisant de celles-ci d'énormes entités administratives urbaines ${ }^{105}$. Les deux premières fusions communales imposées concernaient des entités limitrophes entretenant de longue date des solidarités et des échanges micro-locaux. Elles visèrent à constituer des ensembles d'un minimum de 500 foyers d'abord, puis de 8000 habitants. Mais, tant par les acteurs, les modalités, l'envergure et les enjeux concernés, la dernière en date - réforme structurelle qualifiée de mesure de 《 rognage des régions 》 (cbibō no kirisute saku 地方の切り捨て策) ${ }^{106}$ — est d'un tout autre type. Dans le cas de Sasaguri, le refus de fusionner avec d'autres municipalités pour former une seule méga-entité administrative, peut sans doute être vu comme l'expression d'une résistance locale, qui distingue cette étape des deux précédentes dans les rapports du local au national.

Dans cette double dynamique de dé-/restructuration combinée, les propriétés de montagnes-forêts de Sasaguri ont été soumises à deux mouvements opposés. Le premier est celui d'une privatisation s'intensifiant avec le temps, avec les ventes successives des forêts domaniales de l'État aux communes, puis de celles-ci aux particuliers. Le second est une résistance locale visant à perpétuer, de diverses manières, la forme communautaire des propriétés et de leur gestion. Début Meiji, la superficie des montagnes-forêts des villages composant la commune actuelle (moins Naijū) était donc de $1378,46 \mathrm{ha}^{107}$ ( $1390 c h \bar{o}$ ), dont environ 730 ha de forêts gouvernementales et 485 ha de forêts et landes villageoises. Si les propriétés villageoises apparaissent, elles ne constituaient alors que $40 \%$ de l'ensemble et étaient peu boisées. En 1968, les forêts domaniales s'élèvent à 690 ha, celles de la commune à 499 ha, celles des coopératives à 233 ha et celles des particuliers à I 388 ha, soit une totalité de $2800 \mathrm{ha}^{108}$. Ces chiffres témoignent de la progression importante des plantations forestières, dont l'étendue a doublé en à peine un siècle ; mais aussi de la diminution des forêts domaniales alors que celles des particuliers atteignent la somme des montagnes-forêts possédées 88 ans auparavant par tous les villages réunis. On retrouve aujourd'hui la constance de ces chiffres (totalité de 2395 ha ; dont $\mathrm{I} 376 \mathrm{ha}$ de propriétés particulières) et de ces tendances (diminution des forêts domaniales). L'éloignement contemporain des propriétaires par rapport à leurs forêts semble remettre en question ce processus de développement de la propriété forestière privée. Néanmoins, on peut se demander si la réévaluation très récente de ces territoires sera ou non capable d'entraver ce désintérêt souvent synonyme d'abandon. Enfin, lors de l'examen de ces chiffres, l'attention est attirée par l'existence de 6I3 ha

I05. Le statut de ville n'est plus obtenu qu’à partir de 10000 habitants. Yano Tatsuo 知野 達雄, “Shichōson gappei to iri.ai rin.ya 》市町村合併と人会林野, dans Miyazaki Yoshirō 宮崎幹朗 (éd.), Ebime-ken ni okeru shichōson gappei no tenkai to tenbō 愛媛県における市町村合併の閧開と展望, Matsuyama 松ll (Ehime-ken 愛媛県).

I06. Yano Tatsuo, ibid., p. 252.

107. Chiffres exacts du relevé dans Nishi Nihon Bunka kyōkai (éd.), Fukuoka-ken chiri zenshi I, vol. 22, p. $45^{8-501 . ~ I l s ~ s o n t ~ c i t e ́ s ~ e ́ g a l e m e n t ~ d a n s ~ S a s a g u r i ~ c h o ̄ s b i, ~ p . ~ 218, ~ m a i s ~ l e s ~ c h i f f r e s ~ e ́ t a n t ~}$ arrondis, les sommes y sont légèrement inférieures.

Io8. Minami.ishi Takeshi, Kasuya yōroku, p. 252. 
de propriétés collectives, toutes composantes réunies, qui représentent actuellement $30,8 \%$ des forêts privées et $25,5 \%$ de toutes les forêts.

Dépossession, remembrements, nationalisation, combats pour la récupération et changements des propriétaires, toutes ces étapes des changements et de la complexification de la propriété forestière à Sasaguri se sont faites sous l'impulsion des grands mouvements de réorganisation nationale et locale du territoire depuis la fin de l'époque d'Edo. À ce titre, elles témoignent de la force d'impact du " dehors» sur les réalités internes des communautés locales et sur la notion même de propriété, reformatée sous la contrainte des politiques et des règlementations des pouvoirs régionaux ou centraux. Mais en réponse à ces dernières, toute une gamme de stratégies locales a également été déployée.

\section{À l'articulation des dynamiques du dedans et du dehors : plusieurs configurations locales de gestion pour les espaces forestiers}

En examinant les dispositifs de gestion et d'exploitation collectives des forêts qui révèlent un certain nombre de ces stratégies, mon attention se portera plus particulièrement sur les lignes de continuité entre les modes de relation actuels et le rapport communautaire ancien aux forêts.

\section{Des coopératives de propriétaires ${ }^{109}$}

On constate tout d'abord qu'à Sasaguri les coopératives forestières gèrent $72 \%$ (I439 ha) de toutes les forêts privées (I 989 ha). Le nombre des membres de ces coopératives s'élève à I 239. Ceux-ci se répartissent entre les propriétaires particuliers (détenant 958 ha soit $70 \%$ de l'ensemble des 1376 ha des propriétés particulières) et la commune $(283 \mathrm{ha})$, et les secteurs patrimoniaux (I98 ha) ${ }^{110}$. C'est-à-dire, qu'en dehors d'un certain nombre de propriétaires particuliers, et du bureau des forêts du département de Fukuoka et de l'État, qui gèrent respectivement 132 ha (situés à Haginoo, Naka-machi 中町) et 406 ha (situés à Wakasugi, Yamate et Kido) ne représentant que $5,5 \%$ et $17 \%$ des espaces forestiers de Sasaguri, tous les propriétaires sont associés en coopératives (kumi.ai 組合) pour gérer et exploiter leurs bois (soit eux-mêmes soit en demandant à des tiers). Bien plus, pour l'exploitation de leurs forêts, le département et l'État font aussi appel aux coopératives locales, qui, dans les faits, entretiennent et gèrent donc directement toutes les forêts de la commune.

109. Shinrin kumi.ai 森林組合. Le terme a été traduit en anglais généralement par « cooperative ", mais aussi par « association » (cf. Matsushita Koji et Hirata Kunihito, "Forest Owners' Associations », dans Iwai Yoshiya (éd.), Forestry and the Forest Industry in Japan, zooz. Matsushita Koji, « Recent Problems and New Directions for Forest Producer Cooperatives Established in Common Forest in Japan ", dans Diez J. Julio (éd.), Sustainable Forest Management. Case Studies, 2012.

IIo. Chiffres de février 2012 (mairie de Sasaguri). 
Les coopératives sous une forme ou une autre ont existé dans chaque quartier ${ }^{111}$. $\mathrm{Au}$ tout début du $\mathrm{xx}^{\mathrm{e}}$ siècle, elles se sont multipliées en effet dans le département, suite à l'incitation des autorités départementales à mutualiser les moyens financiers, matériels et financiers ${ }^{112}$. Mais il faut relier ceci à l'importance des structures collectives locales antérieures qui leur servirent d'ancrage. Aujourd'hui, on distingue trois grands types de coopératives qui gèrent soit des propriétés individuelles (qui ne seront pas abordées ici), soit des biens communautaires à l'échelle d'un quartier (ancien village), soit des espaces forestiers dont la propriété terrienne et la propriété arboraire, et éventuellement l'exploitation, sont aux mains de deux ou trois titulaires différents qui peuvent être des groupes de particuliers et/ou des organismes publics.

- Parmi les coopératives qui gèrent des forêts collectives, on trouve celles de Haginoo, Kana.ide et Wakasugi

Haginoo : la «Coopérative de production 》Seisan kumi.ai 生産組合 (qui a succédé il y a une vingtaine d'années à la "Coopérative de production forestière "Seisan shinrin kumi.ai 生産森林組合), à personnalité juridique, gère et exploite une propriété collective qui correspond aux anciens communaux. Ceux-ci, originellement appelés " montagne où l'on entre ensemble " (iri.ai yama 入会山) ou " montagne commune » (kyōōo yama 共同山), puis à partir de Meiji « montagne collective » (kyōyū yama 共有山), sont la propriété de tous les habitants de Haginoo, même si certains habitent aujourd'hui à l'extérieur. Ces terres étaient autrefois des « lieux d'herbes à couper » (kusakariba 草刈り場) formés principalement de landes et de pentes non cultivées. Aujourd'hui elles sont plantées en conifères.

Kana.ide : la «Coopérative des forêts 》 (Airin kumi.ai 愛林組合 ${ }^{113}$ ) gère la propriété collective (plantations de conifères et de feuillus avec rotation de coupes pour la vente), située à l'est du village au-dessus de la forêt du sanctuaire, au nom de tous les membres de la coopérative (40 maisons de propriétaires terriens et forestiers de Kana.ide). Mais aujourd'hui l'exploitation est quasiment abandonnée par ses membres suite à la dévalorisation du bois.

Aujourd'hui disparues, des coopératives de même nom (airin kumi.ai) ont existé à Takata (jusqu'en 2005), à Sannō et à Kido. En cela, Sasaguri est aussi inscrit dans l'histoire nationale. Ayant en effet pour arrière-plan un mouvement national de

III. Les premières coopératives forestières japonaises remontent à la fin du XIXe siècle. Et on verra que Sasaguri a été parmi les premières municipalités a en avoir, puisque celle du canton de Kasuya fut créée en I892. Or selon Matsushita Koji \& Hirata Kunihito, ibid., p. 42, la première aurait été fondée en 1885 dans le département de Toyama 富山. Mais l'essor de ce type de coopératives date de la Loi sur les forêts de $1907\left(\mathrm{n}^{\circ} 43\right)$, dite Seconde loi forestière, qui amendait la Première loi de i897. Selon le recensement ministériel de 2010, il existe aujourd'hui 2261 coopératives forestières au Japon (Ministry of Agriculture, Forestry, and Fisheries, Annual Report on Forest and Forestry, Tōkyō, 2012, p. 31 ; et site du Rin.yachō Heisei 24 nendo sbirrin ringyō bakusho 平成24年度森林・林業白書.

I12. Chikushino shishi hensan iinkai 筑紫野市史編策委員会, Cbikushino shishi 筑紫野市史 民俗編，1999， p. 902.

113. Littéralement : « de nos forêts bien-aimées ». 
reboisement ${ }^{114}$ - lui-même inscrit dans le nationalisme de l'époque - de telles coopératives forestières se sont créées dans tout le pays à partir des années 1925 .

Wakasugi représente un cas particulier qui sera analysé plus loin. À Meiji, les communaux de l'ancien village de Wakasugi sont restés pour une part biens collectifs et pour une autre sont devenus la propriété du sanctuaire Taiso-gū situé sur cette montagne. Mais la gestion et l'exploitation de l'ensemble sont faites par l'association Reihōkai ("Association du sommet sacré 》 霊峰会) dont sont membres toutes les familles de l'ancien village ( 50 maisons au début du $\mathrm{xx}^{c}$ siècle, aujourd'hui 47 ).

Dans tous ces cas, propriété, gestion et exploitation sont communautaires et non séparées, mais chacune des maisons des anciens villages est nominativement propriétaire et membre de la coopérative. Centrée sur l'organisation et les besoins locaux, cette forme de coopérative se situe dans le prolongement direct des pratiques liées aux communaux gérés anciennement par chaque village. La caractéristique en est la continuité des travaux collectifs (débroussaillage annuel, entretien des arbres, coupes, etc.). Mais l'organisation de type coopératif, en formalisant les modalités communautaires anciennes, y a introduit des normes et des valeurs supralocales, tant au niveau de l'exploitation que de la vente.

\section{- La coopérative de patrimoine cantonal}

La "Coopérative de patrimoine du canton de Kasuya " (Kasuya-gun zaisan kumi.ai 糟屋郡財産組合) ${ }^{115}$ a été créée en 1890 (Meiji 23) pour gérer les forêts cantonales de Kasuya (451,50 ha), copropriété de six communes (Sasaguri, Shime, Sue, Hisayama, Kasuya, Umi) et une ville (Fukuoka) du canton de Kasuya, sur le territoire desquelles elles sont plantées. Le centre de gestion se trouve à Sasaguri, car les forêts de Sasaguri en sont à l'origine et sont les plus étendues (201,05 ha à Arata, principalement plantés en cryptomères, certains ayant entre roo et 300 ans). L'historique de cette organisation collective est représentatif d'un aspect du processus de transformation moderne du rapport aux forêts, tel qu'il a dû se construire dans la tension entre les besoins locaux et les législations régionales et nationales successives ${ }^{116}$. Il s'agit d'une longue entreprise jalonnée de combats commencée au XVIII' siècle par Nagahama Shichirō Dayū 長浜七郎太夫 dont, au commencement de cette contribution, je citais les paroles d'affliction devant les montagnes dévastées de Sasaguri. Ces pentes « chauves » situées à Arata (Sannō) et Gō-no-haru (Kido) faisaient partie des terres possédées par le fief,

114. Airin undō 愛林運動 et airin shisō 愛林思想. Mouvement et idéologie à l'origine de la création d'un « jour national de la forêt » en 1934 (airinbi 愛林日) célébré chaque année depuis cette date le 3 avril de façon nationale, qui fut inspiré de l'arbor day lancé par l'Américain J. Sterling Morton qui visita le Japon en 1883 .

115. Appellation abrégée pour "Coopérative du patrimoine de la commune de Sasaguri et autres, soit une ville et six communes du canton de Kasuya " (Kasuya-gun Sasaguri-chō boka isshi roku chō zaisan kumi.ai 糟屋郡篠栗町他一市六町財産組合).

I16. D'après les documents : «Présentation de la coopérative 》 (Kumi.ai no gaiyō 組合の 概要), document interne de la coopérative ; le texte des stèles commémoratives du sanctuaire du Yama no kami 山の神 (dieu de la montagne) d'Arata entretenu par la coopérative cantonale, et le texte "Historique de la montagne communautaire " (Kyōyū-san no yurai 共有山の由来) (cité également dans Sasaguri chōshi, p. 220-227). 
sur lesquelles les habitants avaient des droits d'usages communautaires. En II ans, à partir de 1737, Nagahama Shichirō planta avec ses propres finances 65200 cryptomères et pins. En cela il poursuivait le travail commencé une soixantaine d'années auparavant par son ancêtre Nagahama Shichirō.emon Taketoshi 長浜七郎右衞門武利, guerrier-paysan (gōshi 郷土). Quarante ans plus tard, cette initiative fut continuée sur cinq espaces montagneux de Sannō et Yamate ${ }^{117}$, avec les fonds du fief Kuroda, par Hirai Seijirō 平井清次郎, guerrier de Sasaguri nommé préfet des montagnes du fief. Les autorités féodales de Fukuoka avaient en effet engagé, depuis le milieu du XVII ${ }^{e}$ siècle, une politique de surveillance et d'entretien des forêts sur tout leur territoire. Cette campagne de plantation, commencée en 1784 avec lc concours des habitants de 48 villages du canton, mais principalement ceux de Sasaguri, aboutit en 1799 à la plantation d'une totalité de 1045839 arbres (cryptomères, cyprès, pins) ${ }^{118}$. À la suite de quoi, par un document officiel de 1789 (Tenmei 天明 9), le fief reconnut aux villages qui avaient participé à l'entreprise, et dont les habitants continuaient à entretenir cette forêt, le droit de faire des coupes d'éclaircies dans ces plantations et d'en utiliser les bénéfices de vente pour assurer leur subsistance en cas de disette ou situation de crise ${ }^{119}$. Ces villages purent ainsi sortir de la crise économique locale dans laquelle les avait plongés une série de catastrophes socio-environnementales entre 1828 et $1869^{120}$. Ce document permit également d'obtenir de l'administration de Meiji que ces espaces forestiers (devenus terres d'État en 1868) acquièrent le statut de copropriété cantonale privée, lorsqu'en 1875 , lors de la réforme des taxes foncières (Meiji 8), celui-ci voulut les partager en forêts domaniales et en terres à vendre à des particuliers. Cette bataille autour des statuts : domanial, privé-particulier et privécollectif fut âpre. Et ce d'autant plus que, pour les habitants de Sasaguri, elle se doubla d'un conflit avec les villages du canton de Kasuya à propos de la participation à la copropriété. Dans ces turbulences, des acteurs locaux eurent un rôle capital. Ainsi les pourparlers avec l'État et le canton purent aboutir favorablement pour Sasaguri grâce à un acteur local de premier plan, Tō Kinsaku 藤金作 (1844-1933), responsable du village (shōya 住屋), lui-même propriétaire de forêts et négociant en bois, ainsi qu'homme politique de carrure nationale (sept fois élu membre de la chambre des représentants au Parlement) qui s'occupa notamment des forêts domaniales ${ }^{121}$. En 1876, le statut de terre privée, en tant que « montagne cantonale » (gunzan 郡山) fut acquis, et en 1879 fut signée une convention de partage entre Sasaguri et le canton, dans laquelle, afin de protéger les ressources en eau, les deux parties s'engageaient

I17. Ja-ga-tani 蛇谷, Kaya-ga-tani 行力谷, Kome no yama 米のll!, Hiyamizu-dani 冷水行, Taniguchi 行ll. Dans ce contexte, l'ensemble est appelé «montagne de Sasaguri ».

I 8. D'autres compilations locales non publiées des archives des familles Nagahama et Hirai donnent pour ces plantations les chiffres suivants : à partir de 1670, Nagahama Shichirō Uemon Taketoshi : 70000 cryptomères, 70000 cyprès ; à partir de 1737, Nagahama Shichirō : 1650.00 cryptomères ; à partir de 1784, Hirai Seijirō : 1045839 cryptomères (Kurose Shigefumi, Wakasugi yama ösugi bunpu chōsa, daigoji đi杉l1大スギ分布調第5次, 2009, p. 3).

119. Sasaguri chöshi, p. 222.

120. De mauvaises récoltes causées par le typhon de 1828 (Bunsei 文政 II), les ravages d'insectes en 1836 (Tenpō 大保 7), la sécheresse de I852 (Kaei 获水 5 ), le mauvais climat en 1869 (Meiji 2).

121. Kiyohara Dabutsurō 消原陀佛䬦 (éd.), Tō Kinsaku ō 藤全作翁, 1935, notamment p. 244-271. 
à ne pas faire de coupes abusives et à replanter après chaque coupe. C'est dans ce contexte que, dès 1876 , un autre acteur local, Kurose Enkichi 黒瀬門吉, fut l'initiateur de la dynamique qui conduisit à la fondation en 1892 de la coopérative de ce patrimoine commun (kyōyū zaisan kumi.ai 共有財浐組合), dont il mit en place les règles de gestion. L'objectif était de protéger cette montagne-forêt comme un bien communautaire en imposant une gestion faite à partir de délibérations collectives, notamment à propos des coupes et des partages de bénéfices. Un lieu de surveillance fut implanté dans la montagne à Ja-ga-tani 蛇ヶ谷. Les forêts furent ainsi surveillées en permanence et les plantations furent régulièrement étendues. Avec les transformations administratives successives, les copropriétaires passèrent de 48 villages du canton, à II, puis à 9 communes et villages, enfin, en 1958 , à l'état actuel d'une ville et six communes (toutes ces entités étant représentées au conseil d'administration à chacun de ces stades). Aujourd'hui, le bureau de gestion de Sasaguri emploie pour l'administration trois personnes (respectivement de Sasaguri, de Sue et de Hisayama) et neuf autres pour les travaux forestiers (deux de Haginoo, une de Sasaguri, quatre de Wakamiya 若宮 et deux de Hisayama). La volonté actuelle est de retourner à une conception à long terme de la foresterie, refusant les objectifs économiques immédiats et réévaluant la forêt comme un capital social.

Le point de départ de cette coopérative cantonale est le même que celui des coopératives du type précédent (Haginoo, Kana.ide, Wakasugi). Mais, à la différence de celles-ci restées centrées sur le micro-local, la coopérative cantonale s'est construite à partir d'une double tension entre acteurs locaux (de Sasaguri et du canton de Kasuya) et entre ceux-ci et les autorités féodales d'abord, puis nationales à partir de Meiji. C'est l'intervention, aux niveaux politique, économique et administratif, de personnalités locales se situant à l'interface de tous ces registres qui permit d'assurer la conservation locale de la propriété et de sa gestion, en obtenant, de la part des autorités, le changement de statut des forêts, et, de la part des habitants, la modification de leur conception même des pratiques forestières. En outre, les membres de ces deux types de coopératives sont à la fois propriétaires forestiers, bien que de statuts distincts, et également gestionnaires et exploitants, bien que l'intérêt et la participation soient directs pour les premiers, mais indirects et délégués pour les seconds.

\section{Gestion collective et communaux}

- Les «forêts à bénéfices partagés» (bunshūrin 分收林) et l'《 unification des landes et forêtts» Entre les deux formes de coopératives précédentes, il existe un autre type d'organisation qui se situe de façon différente à l'articulation du micro-local et du régional/national. Ce sont les coopératives qui gèrent les «forêts à bénéfices partagés ». Ce mode de gestion repose sur la distinction des propriétés foncières et arboraires, dont les propriétaires respectifs sont différents et se partagent les revenus des ventes du bois selon un pourcentage convenu (à Sasaguri, de $30 \%$ pour le tréfoncier, et $70 \%$ pour le propriétaire des arbres, mais selon les quartiers le pourcentage peut varier). Le propriétaire terrien en est la commune, et les propriétaires des arbres, qui en sont aussi les exploitants, sont les quartiers dont les habitants sont réunis en coopératives 
de gestion-exploitation. De telles coopératives ne subsistent actuellement que dans les quartiers de Naka-machi, Shimo-machi 下町, Wakasugi, alors que, auparavant, des parcelles gérées de cette façon existaient un peu partout. Mais aujourd'hui, suite à la crise du bois, les villageois ont tendance à se détourner de ce système. Dans ce cas, gestion et exploitation reviennent alors au tréfoncier, c'est-à-dire à la commune ${ }^{122}$. Aujourd'hui la superficie des forêts gérées selon ce système s'est réduite à 31,33 ha.

Le système des forêts à bénéfices partagés est né et s'est répandu dans le pays au cours des restructurations successives des communaux depuis la fin du XIX ${ }^{c}$ siècle. À Meiji, au début de la réforme des taxes foncières (1875), les anciens communaux (iri.ai-chi 入会地), ainsi qu'un certain nombre d'espaces de forêts et de landcs, sont d'abord devenus la propriété collective de chacun des villages (mura kyōyu-cbi 村共 有地) dont les habitants avaient eu jusque-là l'usage des terres. Mais la réunion de ces villages dans les deux unités administratives englobantes (o..aza 大字, " grande circonscription ») de Sasaguri et Seto en I889, puis la mesure nationale d'« unification des forêts et landes propriétés des villages » (buraku-yū rin.ya tōitsu 部落有林野統一) lancée en I910, portèrent atteinte à la gestion des communaux. Jusque-là, celle-ci était en effet restée communautaire et aux mains de ses usagers, sur le modèle antérieur à Meiji. Les enquêtes cadastrales menées par le gouvernement au début de Meiji avaient en effet mis en lumière l'importance de la superficie de ces communaux. Et cette mesure d'" unification "visait en fait à faire récupérer les communaux par les entités administratives englobant plusieurs villages pour en faire cesser la gestion communautaire, et transformer ceux-ci en un capital foncier et forestier, comme support des finances de ces municipalités. Pour faciliter le processus, face aux mouvements de résistance des habitants contraints à un abandon de leurs droits communautaires, des subventions gouvernementales furent accordées aux villages qui acceptaient cette perte. Les villages qui composaient Sasaguri et Seto n'abandonnèrent pas à ces unités administratives toutes leurs forêts et landes communautaires, dont une partie, comme on l'a vu précédemment, a subsisté jusqu'à aujourd'hui sous la forme de propriété collective des habitants. Ces terres représentaient une surface importante. Ainsi, avant cette « unification »-cession à l'unité administrative de Sasaguri, constituée en I889 comme ō.aza par la fusion des quatre villages Sasaguri, Kana.ide, Takata, Haginoo, ces derniers possédaient une totalité de $83,56 c h \overline{~ d e}$ forêts et de 590,72 chō de landes et communaux. (Cette propriété du village était bien sûr inférieure à l'ensemble de l'étendue de forêts et landes existant sur leurs territoires respectifs, puisqu'il y avait d'autres formes de propriétés depuis Meiji) ${ }^{123}$. Par rapport à la totalité de l'étendue

122. La forme inverse existe également. Les «forêts plantées par l'État " (kankō zōrin 它行 造林) sur d'anciens communaux devenus propriétés des grandes entités administratives (dans ce cas les pourcentages sont inversés). Dans ce cadre, l'État a fait des plantations dès 1923 sur 130 chō de Sasaguri. Cf. Kasuya-gun yakusho 糟屋郡役所 (éd.), Kasuyagun-shi 糟屋郡志, 1972 ( $\mathrm{I}^{\mathrm{rc}}$ éd. 1924), p. 402. Ce système a existé ici jusqu'à la fin du Xxe siècle, mais le département est devenu l'exploitant (kenkō zōrin 県行造林). Les bénéfices sont alors partagés à parts égales.

123. Les chiffres de ce paragraphe viennent du document manuscrit Buraku-yū rin.ya tōitsu ni kansuru keii 部落有林野統一に関する経䋘 (date non indiquée mais estimée vers 1930) communiqué par la mairie de Sasaguri, et Kasuya-gun yakusho, ibid., p. 399-408. 
des forêts (I I43,07chō) et landes $(868,02 c h \bar{o})$ situées sur le territoire des 4 villages de l'ō.aza Sasaguri, ces propriétés des villages représentaient respectivement seulement $7,31 \%$ de la totalité des forêts, mais $68,05 \%$ de la totalité des landes et communaux. Ce sont ces derniers espaces, devenus alors incultes, qui étaient au cour des conflits et des négociations. D’un côté, ils étaient l'objet des stratégies développées par les habitants des villages dans le but de conserver leurs propriétés et droits d'usage et, de l'autre, ils étaient visés par les politiques imposées d'une " capitalisation » communale par plantations systématiques. Ceci était encore complexifié par le fait que les mêmes espaces pouvaient avoir été utilisés de façon coutumière par plusieurs villages, dont certains se trouvaient désormais hors des nouvelles limites administratives de Sasaguri et de Seto. Finalement, de ces forêts et landes des villages furent cédés $723 c h \bar{o}$ à Sasaguri en 1920 et I25 chō à Seto en 192I. C'est ainsi qu'en 192I, la propriété de forêts et landes de Sasaguri (en tant qu'ōaza entité administrative) s'éleva à I 407 chō, et celle de Seto à 17,3 chō. Cette récupération communale se fit à titre gratuit, mais avec un certain nombre de conditions. La première d'entre elles fut le contrat des forêts à bénéfices partagés. Établi entre l'unité administrative concernée (Sasaguri et Seto) et les villages, il instaurait un droit de propriété arboraire aux villageois pour ces espaces sur lesquels ils faisaient des plantations et entretenaient des bois de feuillus autorégénérés. Sur les terres non englobées dans ce contrat était accordé, moyennant finances, un droit de collecte d'herbes à fourrage, fruits sauvages, champignons, etc. Des coopératives furent alors créées pour exploiter ces forêts à bénéfices partagés. Bien qu'initiées par les autorités publiques, celles-ci furent adoptées par les villageois comme une forme de parade collective moderne à la volonté gouvernementale de supprimer les organisations communautaires.

Paradoxalement, on retrouve en effet ici, dans des cadres législatifs et économiques tout à fait différents et avec des objectifs distincts, le principe de séparation de la propriété et de l'exploitation-usage qui existait à l'époque féodale. La forêt à bénéfices partagés a pu ainsi être vue comme une forme modifiée des anciens communaux, iri. $a i^{124}$. L'« unification» des terres communautaires se poursuivit et, à chaque étape des fusions communales, la propriété passa à l'entité administrative englobante (en 1927 à Sasaguri devenue commune, Sasaguri-machi, avec ces mêmes 4 villages, puis en 1955 à Sasaguri-chō résultant de sa fusion avec Seto) ${ }^{125}$. Mais les terres gérées collectivement n'ont jamais disparu à Sasaguri, ce qui montre bien l'attachement à cette modalité de rapport collectif aux forêts et aux landes. La mise en ouvre du système des forêts à bénéfices partagés met ainsi en lumière tous les problèmes à l'arrière-plan de la politique d'« unification » des terres communautaires, prises entre deux dynamiques

124. Yano Tatsuo, "Shichōson gappei to iri.ai rin.ya », 2007, p. 259.

125. En outre, depuis la chute des prix du bois, l'explosion du secteur tertiaire et l'augmentation du nombre des habitants travaillant à l'extérieur de Sasaguri, et ce surtout depuis I998, la gestion ou même la propriété d'un certain nombre de forêts de particuliers dans l'incapacité de s'en occuper ou de collectifs (ainsi, celle de petits espaces forestiers qui étaient au nom des quartiers de Kami-, Naka- et Shimo-machi, Haginoo, Kido, Yamate, Sannō, Kana.ide) ont été remises à la commune. Aujourd'hui, celle-ci gère donc des espaces forestiers de petite envergure disséminés sur tout le territoire communal. 
contraires : celle de la résistance des pratiques communautaires et celle des mesures administratives nationales de fusion des communes visant à supprimer propriétés et gestions communautaires au profit de celles de type public ou individuel. La clef de compréhension des stratégies adoptées ici est à chercher dans la valorisation des anciens communaux, iri.ai, perpétuée par la communauté villageoise, non pas seulement pour des raisons d'économie, mais bien de mode de vie et d'identité locale.

- De iri.ai-chi, " terre où l'on entre ensemble ", à commons " : récréer le communautaire Les effets positifs de la politique nationale de récupération des communaux par les municipalités ont été analysés de longue date par les économistes et les agronomes (gestion rationnelle des forêts assurant le reboisement des espaces laissés en friches, protection contre les catastrophes, préservation des ressources en eau et de la biodiversité, etc.). Mais historiens, légistes, géographes et ethnologues ont également souligné leurs effets négatifs sur la société locale. Car il ne s'agit pas seulement de changements structurels, mais aussi de bouleversements de modes de sociabilité, de sens et de valeurs.

Comme nous l'avons vu précédemment, ces anciens communaux étaient le fondement de l'économie familiale et villageoise autarcique. Ils étaient gérés par l'ensemble de la communauté du village, dont les membres devaient respecter des règles d'usage très strictes pour pouvoir utiliser ces espaces, principalement pour le fourrage et les engrais. Ces règles permirent aussi à des villages, comme Wada, Tanaka, Tsubakuro et Onaka - et à d'autres villages limitrophes, aujourd'hui hors de la commune de Sasaguri - qui n'avaient aucun ou très peu de communaux, d'avoir accès aux communaux de ceux qui en disposaient. Jours et heures de collecte, chemins d'accès, délimitation des espaces à utiliser, quantité et grandeur des produits autorisés, tout y était réglementé par des règles fixées par écrit. Cela n'empêcha pas de nombreux conflits entre villages, notamment lorsque les bornes et les limites finissaient par être perdues.

Les archives villageoises témoignent de tels contentieux, comme par exemple celui qui, en 1672 (Kanbun 筧文 I2), opposa Takata à Tsubakuro au sujet de l'usage de coupes de fourrage accordé de façon coutumière à Tsubakuro sur des communaux déjà partagés entre Takata et Tanaka. Le litige se termina avec la décision prise en commun par les responsables (shöya) des trois villages de fixer, à l'aide de repères, les limites à l'intérieur desquelles chacune des trois collectivités pouvait faire ses coupes. Mais il reprit 150 ans plus tard (1824, Bunsei 文政 7), et cette fois le village de Wada y fut mêlé. Suite à des plantations de pins sur une partie des communaux, la surface ayant diminué et les repères ayant à nouveau disparu, il fallut renouveler la procédure. Celle-ci fut ratifiée par le préfet du canton et ainsi définitivement entérinée ${ }^{126}$. C'est là un exemple, mais les archives des autres villages en contiennent bien d'autres tout à fait similaires ${ }^{127}$.

126. Sasaguri chöshi, p. 182-187 et archives manuscrites Fujiki (Sasaguri shiryo shitsu).

127. Archives manuscrites: Ōtani 大谷 $\mathrm{n}^{\circ}{ }_{1}, \mathrm{Chō}$ 髟 ${ }^{\circ s} 29$ (1872), 62 (1795) (idem, Sasaguri shiryō shitsu). 
Néanmoins ce qui ressort de ceci, c'est, d'une part, la capacité à gérer collectivement, par-delà les conflits inévitables, des ressources naturelles limitées sans que celles-ci finissent par être épuisées ; et, d'autre part, celle de les partager, alors même qu'elles sont restreintes, par le biais d'un accord collectif, obtenu par des négociations menées au nom de l'ensemble de villageois reconnaissant communément les mêmes règles. L'organisation et les règles présidant à l'usage des communaux faisaient partie de ce qui structurait non seulement les pratiques, mais aussi et surtout le vivre ensemble local. C'était aussi une parade au régime de propriété féodal et au manque d'espace et de forêts. De tels faits infirment la théorie de Garriett Hardin sur la " tragédie des communaux ", ou des biens communs, qui seraient nécessairement objets de surexploitation et de dévastation ${ }^{128}$. Cette théorie a eu le mérite de focaliser l'attention sur la part déterminante des relations entre groupes sociaux dans les relations à l'environnement naturel. Elle a été cependant critiquée dès les années 1970 par des auteurs occidentaux et japonais ${ }^{129}$ comme étant biaisée par la confusion entre propriété et usage, et non représentative des multiples réalités locales, donc loin de représenter une fatalité. Bien plus, située à l'opposé du communautaire, c'est plutôt la gestion moderne nationale ou privée, visant la rentabilité à tout prix de plantations monotypes à vaste échelle, sujettes à la fluctuation des marchés et de la demande, qui, actuellement, est accusée d'être la cause directe de la surexploitation de l'environnement et de sa détérioration.

«Les communaux (montagnes à fourrage et à bois), les forêts du village et l'agriculture sans machines impliquaient des droits et des devoirs communs, des travaux collectifs, des réunions, des rites annuels et des loisirs communautaires qui aujourd'hui ont disparu ", reconnaît Matsuo Kenji, responsable de la coopérative forestière de Haginoo. Pour lui, la solidarité du village n'est plus ce qu'elle était, alors même que les habitants extérieurs à ce quartier de Sasaguri considèrent que Haginoo a conservé des liens communautaires particulièrement forts ( danketsu ga tsuyoi » 団結が強い). De fait, Haginoo est, avec Wakasugi, l'un des anciens villages qui ont gardé, sous forme de coopérative, une gestion collective de ce qui fut leurs communaux. C'est là aussi que se trouve une part importante des forêts de la commune. L'inverse est également vrai : moins il y a d'espaces forestiers, et moins il est question de gestion collective. La rupture provoquée par les remaniements administratifs successifs des communaux se situe non seulement au niveau des rapports avec l'environnement et des systèmes de propriété, mais aussi à celui de tous les dispositifs collectifs de gestion et de la relation même à ce " patrimoine »

128. Garrett Hardin, "The Tragedy of the Commons ", Science 162 (1968).

129. En trop grand nombre pour être cités ici, les débats et recherches sur cette question développés dans de nombreuses disciplines depuis la théorie de Hardin, ont été repris et synthétisés dans des travaux récents dont ceux de F. Berkes et al., "The Benefits of the Commons ", Nature 340 (1989) et « The Tragedy of the Commons: Twenty-Two Years Later », Human Ecology I8, vol. I (1990), (où l'on trouve une bibliographie très complète); et Miya.uchi Taisuke 宮内 泰介, «Komonzu no shakaigaku. Shizen kankyō no shoyū, riyō, kanri wo megutte » コモンズの 社会学——自然環境の所有・利用·管理をめぐって, dans Torigoe Hiroyuki 鳥越皓之 (éd.), Shizen kankyō to kankyō bunka 自然環境と㻴境文化, 200I. 
à la fois matériel (spatial et végétal), structurel, culturel et social. Tout ceci met bien en lumière le poids décisif du lien entre modalités du vivre ensemble local et préservation des espaces forestiers, et l'impact réciproque des bouleversements qui les touchent.

La disparité qui existe sur ce point à l'échelle de la commune de Sasaguri se retrouve partout ailleurs. Suite aux fusions communales et notamment à celle de Heisei en cours, quatre grands types de solutions semblent aujourd'hui possibles : la conservation de la gestion communautaire de ces terres restées propriété du groupe local ; le retour à la communauté villageoise des propriétés devenues individuelles auX XIX ${ }^{e}$ et $\mathrm{XX}^{e}$ siècle ; la création de secteurs patrimoniaux ; la totale disparition des formes collectives de propriété (avec ou sans instauration d'un droit arboraire) sur terres et forêts qui sont soit partagées en propriétés individuelles, soit récupérées par les municipalités ayant fusionné. Les deux premières sont en diminution tandis que la dernière est en augmentation ${ }^{130}$. Mais ceci concerne le foncier sur le plan du droit. Or, comme nous l'avons vu avec les différents types de coopératives et autres modes collectifs de gestion forestière, il existe en fait de multiples possibilités de combinaison de ces trois paramètres : propriété, gestion et exploitation. Et ces combinaisons ont pour arrière-plan une ambiguïté sur la notion de "bien commun " qui remonte aux usages coutumiers des anciens communaux.

C'est ce que mettent aussi en évidence de récents travaux. Commencées dès Meiji, notamment par les juristes, les agronomes et les économistes ${ }^{131}$, les études sur les communaux et leurs transformations sont très nombreuses. Mais les plus récentes dans le champ ethnologique et sociologique ${ }^{132}$ réexaminent leur histoire et leurs réalités en attirant l'attention sur cette ambiguïté. Pour ce faire ils utilisent la notion « commons » ${ }^{133}$ (komonzu コモンズ) comme équivalent de la notion-clé sōyū 総有 《 le bien communautaire »- différent de l'acception qui est faite du terme sōyū par le droit moderne - et défini comme étant « la chose de tous/ce qui appartient

130. Yano Tatsuo, «Shichōson gappei to iri.ai rin.ya » et Mitsumata Gaku 三俣学, «Shichōson gappei to kyūson zaisan ni kansuru ichi kōsatsu. Kankyō hozen-komyuniti saikō no jidai no shichōson gappei no giron ni mukete 》市町村合併と旧村財産に関する一考察——環境保全・コミュ 二ティ再考の時代の市町村合併の議論にむけて, Nibon minzokugaku 日本民俗学 245 (2006) (numéro spécial de la revue des études d'ethnologie du Japon consacré à la fusion des communes).

131. Pour une bibliographie des ouvrages anciens, voir par exemple Furushima Toshio (éd.), Nibon rin.ya seido no kenkyü.

132. Akimichi Tomoya 秋道智洂, Komonzu no chikyūshi. Gurōbalu-ka jidai no kyōyūron ni mukete コモンズの地球史一一グローバル化時代の共有論に向けて, 20Io. Miya.uchi Taisuke 宮内泰介 (éd.), Komonzu wo sasaeru shikumi コモンズをささえるしくみ, 2006. Ino.ue Makoto 井上真, Miya.uchi Taisuke (éd.), Komonzu no shakaigaku. Mori, kawa, umi no shigen kyōdō kanri wo kangaeru コモ ンズの社会学———森・川・海の資源共同管理を考える, Shirīzu Kankyō shakaigaku シリーズ罢境社 会学 2, 2001. Nishida Yoshikazu 西田彦一, Iri.ai rin.ya to sbüben shakai 入会林野と周辺社会, 2009.

133. De l'anglais commons. Notion définie comme désignant les ressources dont la gestion et l'usage sont partagés entre une pluralité de sujets, ainsi que ce système de gestion et d'usage (Suga Yutaka 菅豊, “Komonzu to seitōsei »コモンズと正当性, Kankyō shakaigaku kenkyū 環境社 会学研究 2 (2005), p. 24). 
à tous " (minna no mono みんなのむの) ${ }^{134}$. Cette notion d'appartenance est au cour de l'ambiguïté car, avant de désigner une propriété - ce que n'étaient pas les communaux avant Meiji pour les communautés locales - elle signifie un lien territorial partagé dans les usages communautaires. Kyōyu 共有, "le bien commun/collectif », autre notion-clé, est à l'articulation entre le sōyū et la notion de propriété moderne. Cette dernière, l'ethnographie le prouve, peut être contaminée par l'ambiguïté du communautaire (groupe au-delà des individus/ensemble non partageable, où la totalité est insécable) qui n'est pas le collectif (ensemble d'individus/d'éléments individuels séparables). En effet, dans les villages, la propriété forestière particulière reste bien souvent aujourd'hui encore considérée comme continuant à faire partie de ce «bien de tous ", et, dans les faits, il peut être difficile pour un particulier d'en disposer librement. La question posée ici est celle de la possibilité d'action et des droits du groupe et de l'individu par rapport au communautaire et au collectif. Et certains soulignent que ce «bien communautaire » assurait le «droit de vivre des "faibles" ${ }^{135}$. Le grand apport de ces recherches est la mise en lumière de toutes les variabilités des modalités des usages communautaires (directs, partagés, contractuels, etc.) selon les lieux, les groupes et le type de ressources naturelles; ou encore l'éclairage sur le cumul d'usages multi-strates des ressources naturelles des villages (à usages communautaires libres, sous gestion contractuelle villageoise, objet de limitations temporelles ou instrumentales, particulières) qui peuvent coexister dans un même espace-temps ${ }^{136}$. Tous ces travaux, grâce à une riche ethnographie des modes d'accès aux ressources naturelles, de leurs usages et modalités de gestion, montrent en quoi ce «bien communautaire » est défini par l'histoire des relations entretenues par un groupe avec un territoire, histoire qui en retour contribue aussi à définir ce bien communautaire lui-même. À ce titre, le sentiment d'appartenance - avec toute l'ambiguïté quc comportc ccttc notion - cst réciproque (on appartient au village comme son territoire nous appartient) et c'est ce qui différencie ce qui est intérieur au village de ce qui lui est extérieur. Mais il est clair aussi que le communautaire n'est pas seulement une question du «dedans». Il n'a cessé de se construire à l'articulation de celui-ci avec le «dehors » - les pouvoirs en place, les autres collectivités - par le biais de moyens divers qui le légitiment des deux côtés (impôts, accords mutuels ou autre) ${ }^{137}$. Nombreux sont les auteurs pour qui, aujourd'hui, l'écrasement du communautaire tel que les autorités l'ont longtemps pensé n'est plus à l'ordre du jour, et pour qui le bien commun est à revaloriser ${ }^{138}$. La question est ainsi placée au centre de débats

134. Fujimura Miho 藤村美穂, “"Minna no mono” to wa nanika »「みんなのもの」とは何か, Ino.ue, Miya.uchi (éd.), Komonzu no shakaigaku.

135. Torigoe Hiroyuki 鳥越皓之, « Komonzu no riyōken wo kyōju suru mono 》 コモンズの利 用権を享受する者, Kankyō shakaigaku kenkyū 環境社会学研究 3.

136. Kada Yukiko 嘉田由紀子, “Seikatsu jissen kara tsumugi dasareru jūsōteki shoyūkan » 生活车践からつむぎ出される重層的所有観, Kankyō shakaigaku kenkyū 環境社会学研究 3, p. 72-85.

137. Suga Yutaka, "Komonzu to seitōsei », 2005, p. 22-39.

138. Parmi beaucoup d'autres, Mitsumata Gaku, "Shichōson gappei to kyūson zaisan ni kan suru ichi kōsatsu ", 2006. 
où s'affrontent les interprétations du droit moderne et du droit coutumier ${ }^{139}$. Si elle a commencé par les espaces forestiers, la revalorisation ethnologique et sociologique du communautaire ne s'y est pas limitée. Les mêmes problématiques s'appliquent aujourd'hui sur d'autres espaces de ressources naturelles comme les fleuves ${ }^{140}$, la mer $^{141}$, les lacs ${ }^{142}$. Cette approche des sciences humaines et sociales coïncide avec la «vogue » de la «montagne du village » (sato-yama) évoquée précédemment, qui, pour certains auteurs, n'est autre que la version contemporaine des anciens communaux ${ }^{143}$. Et, dans ce mouvement de « redécouverte» du bien commun, on a vu aussi émerger dans les médias et le discours public des expressions, faites sur le modèle de la montagne du village (sato-yama), telles que «mer du village» (sato-umi 里海) ou « fleuve du village» (sato-gawa 里川) pour revendiquer droits et devoirs collectifs envers ces espaces.

La multiplication des structures de gestion collective des espaces forestiers de Sasaguri, qui montre le recours préférentiel et efficace au collectif micro-local, repose aussi sur toutes ces logiques. Or, gestion et usages y ont été également associés à un facteur qui apparaît peu, voire pas du tout, dans les travaux cités précédemment, le facteur religieux. En dernier lieu, j'examinerai la place du religieux dans les rapports communautaires aux forêts.

\section{Forêts, sanctuaire tutélaire et communauté locale : les cryptomères de Wakasugi}

Comme dans tous les autres quartiers de Sasaguri, la gestion et l'entretien des forêts de Wakasugi sont directement liés à la cohésion du groupe local qui s'est construit autour de ces activités. Mais ici, intervient en plus un ensemble de dynamiques ancrées dans le religieux. Je ne veux pas parler des éléments religieux coutumiers liés aux pratiques forestières, qui ne sont pas une particularité de Wakasugi. Ils existent également partout et ce, jusqu'à aujourd'hui. Les arbres étant le support privilégié des divinités, les sanctuaires, comme nous l'avons vu, sont entourés de leurs forêts. Le dieu de la montagne, qui est aussi maître des forêts, est partout objet de célébrations annuelles faites par le groupe local. Et, avant de commencer les coupes forestières, ceux qui en sont chargés n'oublient pas d'accomplir au pied d'un arbre un bref rite d'invocation au dieu de la montagne (yama no kami 山の神), rite valant pour demande d'« autorisation » et de protection, et un autre de remerciement sur

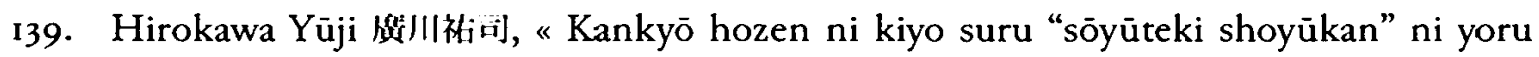
kōkyōteki tochi riyō chitsujo no keisei 》環境保全に寄なすする「総伯的所们観」による公共的土地利用 秩序の形成, Kökyō kenkyū 公共研究 8, vol. I (2012), p. 130-170.

140. Suga Yutaka, "Komonzu to seitōsei » et Suga Yutaka, Kawa wa dare no mono ka. Hito to kankyō no minzoku-gaku 川は誰のものか一人と噮境の民俗学, 2006.

141. Nakamura Hisashi 中村尚司, et al. (éd.), Komonzu no umi コモンズの海, 1995.

142. Kada Yukiko, "Gyorō to kankyō hozen. Biwako no sesshō kindan to gyogyōken wo meguru shinsei no rekishi kara saguru 》漁労と環境保全——琵琶湖の殺生禁断と洦業権をめぐる心 性の禁史から探る, dans Torigoe Hiroyuki (éd.), Shizen kankyō to kankyō bunka, 200I, p. 47-76.

I43. Miya.uchi Taisuke, «Komonzu no shakaigaku. Shizen kankyō no shoyū, riyō, kanri wo megutte ", 200I. 
une souche coupée lorsqu'ils ont terminé. Le religieux en question ici est celui qui a structuré historiquement le groupe social.

De même que Kido, avec le « nouveau pèlerinage des 88 temples », est le pôle religieux majeur de l'ancien village de Sasaguri, Wakasugi représente le pôle religieux historique du côté de Seto. Le pèlerinage de Kido et Wakasugi sont tous deux situés au coeur des espaces forestiers. Mais la fondation du premier remonte à l'époque moderne $\left(1835^{144}\right)$, et il est structuré d'une part par l'action collective de laïcs locaux et d'autre part par l'intensification de la circulation sur l'axe est-ouest reliant Kido à la région périphérique; tandis que, comme on l'a vu précédemment ${ }^{145}$, les structures religieuses de Wakasugi remontent à l'époque ancienne et à des groupes bouddhiques et shugen liés aux pouvoirs politiques et religieux régionaux et nationaux ; quant aux axes de circulation de cette partie sud de Sasaguri, ils sont orientés nord-sud (Satani, shugen du mont Hōman et Daizaifu) et est-ouest (Fukuoka, Hakata, la mer, le reste du pays). Non seulement sanctuaire tutélaire de Wakasugi, mais aussi centre rituel régional (sōsha 総社) depuis la période d'Edo, le sanctuaire Taiso-gū situé dans les forêts au sommet du mont Wakasugi avait la charge d'accomplir, sur demande des autorités féodales ou villageoises, des rites pour la pluie, pour le beau temps et contre les vents, en cas d'aléa climatique mettant en danger les récoltes ${ }^{146}$. Les pèlerins y venaient de toute la région avoisinante. Du début du XVII ${ }^{\mathrm{e}}$ siècle (sur volonté de Nagamasa 長政 [1568-1623], premier des daimyō Kuroda) jusqu'à 1872 (date de l'interdiction du shugendō à Meiji), le desservant de ce sanctuaire fut le yamabushi-moine ${ }^{147}$ de l'Ishii-bō, temple sbugen ${ }^{148}$ situé dans le village de Wakasugi, qui était responsable de la gestion du Taiso-gū en tant que bettō-dera 別当寺149. L'Ishii-bō était aussi le représentant en chef du shugen du mont Hōman pour toute la partie orientale de la sphère d'influence de ce dernier dans la région. Il était en cela assisté par la communauté locale qui entretenait les forĉts dc la montagne. C'est cette structure socioreligieuse ancienne, avec les implications politiques, administratives des pouvoirs féodaux puis nationaux, qui eut une importance particulièrement déterminante pour l'existence même des forêts de Wakasugi. Les conflits autour de

144. Voir dans ce volume Nakayama Kazuhisa, "La dynamique de création, réplication et déclin des lieux de pèlerinage ", p. 29I.

145. Voir ci-dessus p. 124-129, la section "Communautés par-delà les montagnes ", et dans ce même volume de Suzuki Masataka, "Continuités et transformations de la société locale ", p. 29-36, et Mori Hiroko, "Le mont Wakasugi, son sanctuaire et le shugen ", p. 209-215.

146. Cf. les registres des rites annuels tenus par les responsables successifs de l'Ishii-bō 石井坊 de 1693 à 1855 (archives Ishii-bō, nos 109,140 à I58), ainsi que les notifications de rituels entre 1794 et 1891 (archives Ishii-bō, $n^{o s} 227$ à 394) (archives conservées et classées par le Sasaguri shiryō shitsu).

147. Dit aussi moine du sanctuaire, sbasō 社僧 (Cbikuzen no kuni zoku fudoki furoku 筑前国 続風土記付録 cité dans Gōya Takeshiro, Chikuzen Wakasugi kyōdoshi, p. 36).

148. Appellation plus complète : Taiso-zan Ennen-ji Ishii-bō 太祖山延年寺石井坊.

149. Configuration cultuelle mixte, courante jusqu'à Meiji, dans laquelle temple bouddhique, sanctuaire des dieux et organisation shugen sont réunis en une seule structurc constitućc de plusieurs groupes hiérarchisés et articulés entre eux. Ici, le responsable de l'Ishii-bō était le seul et unique desservant shugen pour gérer l'ensemble. Voir dans ce volume Mori Hiroko, « Le mont Wakasugi, son sanctuaire et le shugen ", p. 232-248. 
cette montagne-forêt, notamment, mettent en lumière la façon dont cet élément religieux a joué le rôle d'opérateur efficace.

Pour défendre les forêts de cryptomères contre les coupes abusives ou les appropriations extérieures, les habitants de ce village - situé sur les flancs même de la montagne et vivant principalement des activités forestières - ont toujours eu recours à l'argument de l'histoire mythique de ces arbres (l'acte fondateur de l'impératrice Jingū et le "cryptomère partagé ») et de leur valorisation religieuse historique (Taiso-gū, Oku-no-in et la légende de ses fondateurs prestigieux ; parcours rituel du shugen du mont Hōman), en s'appuyant notamment sur les textes anciens relatant les origines du sanctuaire ${ }^{150}$. Cette légitimité de la protection d'un périmètre attribué au sanctuaire a été reconnue par les daimyō. Dès 1544 , ces droits sont garantis par la promulgation de l'interdiction d'y couper les arbres, imposée par les Ô.uchi 大内 aux villageois et aux guerriers ${ }^{151}$. Les Kuroda l'ont perpétuée. Bien plus, ils n'ont cessé d'entretenir et reconstruire le sanctuaire du sommet ( 23 fois entre 1647 et $1832^{152}$ ) et de faire des donations. Au début du XVII ${ }^{\mathrm{e}}$ siècle, ils ont fait don au Taiso-gū des 3000 tsubo (environ I ha) $)^{153}$ où se dressent les « arbres divins » et où se situent tous les lieux cultuels du sommet (Oku-no-in et sa source d'eau lustrale, etc.). Cette montagne, comme toutes les terres, était en effet propriété du fief. Et les Kuroda se sont employés dès le début du XVII ${ }^{e}$ siècle à protéger les forêts en promulguant des obligations de planter des cryptomères, des interdits sur toute coupe dans les montagnes et sur le gibier. Pour couper un seul arbre, il fallait une autorisation du fief ${ }^{154}$. Toute violation était punie d'exil $^{155}$. Par-delà la protection des forêts du sanctuaire, leurs objectifs étaient très pragmatiques. Ils y prélevaient du bois pour leurs besoins, mais de façon raisonnée et avec replantation immédiate ${ }^{156}$. Les cryptomères étaient réservés à l'usage du daimyō, les cyprès à la construction ou reconstruction des bâtiments du Taiso-gū, et les feuillus pouvaient être utilisés communautairement par les villageois. En outre, grâce à ses forêts, la montagne de Wakasugi constituait une chasse réservée au daimyō, appelée

150. Cbiku no zenshū Kasuyagun Taiso gongen gūki 筑之前州粕屋郡大祖権現容記 (1597), Chikuzen Kasuyagun Wakasugi Taiso jinja engi 筑前糟屋郡分杉大祖神社縁起 (1692), Chikuzen no kuni Omote Kasuyagun Taisozan denki 筑前國表糟屋郡太祖山伝記 (XVIII'), traditions anciennes condensées, fin du Xvir - début XvIII ${ }^{e}$ siècle par Kaibara Ekiken dans le Cbikuzen no kuni zoku fudoki 筑前国続風土記, p. 403). Voir dans ce volume les analyses de Suzuki Masataka, «Continuités et transformations de la société locale ", p. 52-55, et de Mori Hiroko, p. 209-215.

I5I. «Utilisation des arbres et bambous de la montagne Taiso [...] : dorénavant, il est formellement interdit aux hommes armés et à toute personne d'y adopter une conduite violente et d'y couper tout arbre ou bambou, et notamment, les cryptomères, qui sont des arbres "divins" (shinboku) du sanctuaire Taiso, et sont donc particulièrement interdits de coupe ", Sasaguri chöshi, p. 241.

152. Archives Ishii-bō conservées au Sasaguri shiryō shitsu ; dates compilées également dans Kyūshū rekishi shiryōkan (éd.), Chikuzen Kasuya. Wakasugi yama no bukkyô iseki, p. 32.

153. Archives Ishii-bō, $\mathbf{n}^{\circ}$ 37-2. Et Sasaguri chöshi, p. 241 .

154. Archives Ishii-bō, $\mathrm{n}^{\text {os }}{ }_{172}, 175,176,177,178$ (1744-1772).

155. À Meiji, le vol de bois dans la montagne était encore puni de coups de fouet par les gardes (Gōya Takeshiro, Chikuzen Wakasugi kyōdoshi, p. IOz).

156. Kondō Tenji, "Fukuokahan no sanrin ni tsuite ». 
" grenier aux cerfs ${ }^{157}$ " (kakura 鹿倉), que devaient entretenir les villageois, rabatteurs lors des chasses annuelles (et constamment confrontés aux déprédations causées par les animaux en surnombre). À la fin du XVII ${ }^{\mathbf{c}}$ siècle, Kaibara Ekiken écrivait : «Aujourd'hui sur cette montagne (Wakasugi) se dressent de nombreux cryptomères communs (yonotsune 世の常). Etant très grands, ils sont magnifiques ${ }^{158}$. Il précise qu'ils étaient « communs », c'est-à-dire qu'ils n'étaient pas des ayasugi comme l'arbre du premier bouturage mythique. On ne sait si cette espèce de peuplement d'ayasugi a réellement existé à Wakasugi. Car selon les enquêtes ADN récentes, le seul (20 m de haut, $6 \mathrm{~m}$ de circonférence) qui porte aujourd'hui ce nom n'en est génétiquement pas un. Mais ce qui est sûr, c'est que, à part les quelques individus exceptionnels encore debout aujourd'hui évoqués précédemment dans la seconde partie, tous les arbres les plus anciens ont été coupés au XVI ${ }^{e}$ siècle. En effet, comme le relate aussi Ekiken, un siècle auparavant des coupes dévastatrices avaient été faites par le seul des daimyō qui ait violé les interdits, Kobayakawa Hideaki 小早川秀秋 (I582-1602), pour fournir le bois de construction de la flotte avec laquelle Hideyoshi envahit par deux fois la Corée (I592 et I597). Ces arbres poussaient sur le lieu dit « champ des ayasugi », dont la tradition locale attribue la plantation à l'impératrice Jingū. Hideaki fut donc accusé d'avoir coupé les arbres dédiés aux dieux. Craignant leur châtiment (ou étant ensuite tombé malade, selon les traditions), il fit faire des boutures de ces cryptomères et replanter la montagne (mais il mourut jeune et sans descendance, ce qui a été interprété comme un châtiment des dieux). Et c'est ensuite que les Kuroda, daimyō qui lui succédèrent dans le fief de Fukuoka, pratiquèrent une politique de reboisement. Les forêts de Wakasugi de la fin du Xvir ${ }^{e}$ siècle résultaient donc de ces campagnes de plantations d'Edo. Mais, à plusieurs reprises au cours de l'histoire récente, ces arbres déclarés divins ont été à nouveau coupés ou mis en danger par les pouvoirs publics. En effet, la forêt domaniale située sur la ligne de crêtes ouest de la montagne, là où se dressent les plus vieux cryptomères, fit l'objet de coupes importantes durant la guerre du Pacifique (pour la marine) ${ }^{159}$, et depuis l'après-guerre par l'Office des forêts ${ }^{160}$. Pour compenser ces pertes, les habitants firent alors des plantations sur toutes les montagnes qui étaient propriété du village ou de la coopérative cantonale.

157. Shika 鹿 (Cervus nippon) : cerf Sika. Cervidé plus petit que le cerf et le daim, à robe tachetée comme celle du daim, dont les bois peuvent dépasser 8 cors.

158. Kaibara Ekiken, Cbikuzen no kuni zoku fudoki, p. 403.

159. Cf. le texte d'invitation à la cérémonie d'« ouverture des yeux » de la statue de l'oratoire de Kannon de Wakasugi faite par le sculpteur Tominaga Chōdō 富永朝常堂 le 18 avril 1952 : « Si nous avons fait ce projet de sculpter une statue du bodhisattva Kannon en utilisant un cryptomère sans égal dans tout le pays, c'est parce que, déplorant profondément la disparition de nos vieux cryptomères immenses, si rares dans l'ouest du Japon, coupés année après année, nous avons voulu par là donner la possibilité aux générations futures de se souvenir longtemps de l'âme de ces arbres superbes. Nous avons voulu aussi apaiser les âmes de nos morts héroïques qui ont été les précieuses victimes de leur participarion à la seconde guerre mondiale [...] ", Gōya Takeshiro, Chikuzen Wakasugi kyōdoshi, p. 68. Voir aussi dans ce même volume Suzuki Masataka, "Continuités et transformations de la société locale », p. 94.

I60. Gòya Takeshiro, ibid., p. II. 
Ceci est une conséquence de la restructuration foncière de Meiji. Comme partout ailleurs au Japon, où celle-ci donna lieu à de longs litiges et procès pour la récupération des forêts villageoises, les habitants de Wakasugi eurent à se battre pour leurs forêts. En I875, les terres de Wakasugi, ancienne propriété du fief, furent accaparées en partie comme forêt domaniale par l'État, qui partagea le reste entre le village et des particuliers. La saisie de cette forêt domaniale (située au sommet du mont Wakasugi, sur les lieux-dits Gongen 権現, Sugi-daira 杉平, Kagari-daira 籍平, d'une superficie de 94 chō 4 tan $6 s e 28$ bu, environ Ioo chō, soit à peu près Iooha) fut à l'origine d'un triple contentieux : entre Kuroda Nagashige 黒田長成 (1867-1939), descendant des Kuroda qui réclamait la restitution de ces terres en tant qu'héritage familial et propriété privée lui revenant de droit, et l'Office des forêts ; entre les sept villages de Kasuya situés tout autour du mont Wakasugi ${ }^{161}$, qui revendiquaient la nécessité de conserver le statut de forêt domaniale à ces espaces forestiers représentant les ressources en eau indispensables à la vie de leurs 3700 foyers et à l'irrigation de leurs 1500 ha de rizières, et l'héritier des Kuroda ; et entre le village de Wakasugi, réclamant la restitution du périmètre du sanctuaire Haut (Jōgū 上宮), ancienne donation faite aux villageois par les Kuroda, qui avait été intégré par l'État dans la forêt domaniale, et l'Office des forêts ${ }^{162}$. Dans cet affrontement de logiques opposées - nationales, communautaires locales et privées - autour des forêts, deux stratégies furent déployées par les villages. D'une part, contre la privatisation et pour le maintien du statut national de ces forêts, fut mise en avant ce que l'on peut appeler la " communauté de l'eau » existant entre les villages au pied d'une même montagne (par-delà toute limite administrative), et entre ceux-ci et les espaces forestiers dont leurs réserves en eau dépendent. L'argument avancé était que, si la montagne devenait une propriété privée il deviendrait impossible d'empêcher l'abattage des arbres à grande échelle, ce qui mettrait en danger les ressources en eau. D'autre part, contre la saisie par l'État et pour la restitution au village du périmètre forestier du sanctuaire, fut défendue la cause de la communauté cultuelle de Wakasugi. L'argument était que celle-ci se trouvait dans l'incapacité d'accomplir correctement les rites en son sanctuaire tutélaire, car l'enceinte en avait été réduite à $297,52 \mathrm{~m}^{2}$ autour du seul bâtiment principal, alors que le périmètre ancien d'I ha était une donation légitimée depuis le $\mathrm{XVII}^{\mathrm{e}}$ siècle ${ }^{163}$. Les deux lignes de combat avaient le même objectif socio-environnemental : la préservation des forêts du mont Wakasugi, et par là la garantie de toutes les activités des communautés locales. Mais elles reposaient respectivement sur des arguments séculiers et religieux. Pour l'État, ces contentieux n'étaient pas du même ordre, puisqu'il s'agissait d'un côté de conserver 100 ha de forêts comme bien national dont bénéficiaient les communautés locales, et de l'autre d'en restituer i ha à un village. Mais son autorité était en jeu dans les deux cas. Le poids de la légitimité des Kuroda fut utilisé de façon antinomique, contre les villages par Kuroda, et pour le village par

161. À leur tête était Seto, l'unité administrative formée en 1889 par la réunion de 6 villages, dont Wakasugi, que Seto représente dans ce conflit.

162. Sasaguri chöshi, p. 228-229.

163. Archives Ishii-bō $n^{\circ s} 8,16,25,30,37^{-2}$ (don de 3000 tsubo, et mesures du périmètre). 
Wakasugi, mais contre l'État par les deux parties, l'État étant aussi l'arbitre. Dès I89I, des démarches sans résultats avaient été engagées, dont des requêtes adressées au ministre de l'Intérieur et au ministre de l'Agriculture, par l'entremise du préfet. Des archives écrites furent produites des deux côtés ${ }^{164}$. Mais la question locale était contaminée par le procès engagé entre Kuroda et l'État. C'est l'argument religieux qui, le premier, obtint gain de cause, en 1918 après quatre ans de procédure intensive et de démarches collectives jusqu'à la capitale. Alors que le procès de Kuroda Nagashige durait depuis 23 ans sans avancée significative, c'est le village de Wakasugi qui eut gain de cause le premier. En 1914, une action auprès de l'Office des forêts est lancée par le prêtre du Taiso-gū, Sassa Tamotsu 佐々保, assisté de l'ancien desservant du sanctuaire, Ishii Tamaki 石井環, fils du prêtre-moine shugen destitué à Meiji, et deux autres représentants des villageois, tous en tant que fidèles du sanctuaire (ujiko 氏子) ${ }^{165}$. Ici, c'est la force de la solidarité locale autour de ce pôle religieux majeur, qui, dépassant les clivages de séparation des cultes des dieux et des bouddhas imposée par le gouvernement de Meiji, a joué un rôle déterminant. Notamment l'action décisive et conjointe d'Ishii Tamaki, l'ancien prêtre du sanctuaire, et de Sassa Tamotsu, le nouveau. Wakasugi récupéra donc l'enceinte de son sanctuaire. La forêt domaniale fut, quant à elle, finalement préservée en tant que telle, à part cette surface d'i ha restituée aux villageois. Le sanctuaire et sa communauté cultuelle, d'un côté, et de l'autre l'État, eurent le même poids comme garants et défenseurs du bien commun. Cet exemple confirme la force et l'efficacité du religieux dans la défense de la montagne-forêt, lieu d'ancrage ancien des structures de culte. Et ce, à une époque où l'emprise étatique sur le religieux était sévère. En cela, le cas du Taiso-gū est à rapprocher de tous les conflits similaires qui se déroulèrent autour des forêts des sanctuaires au même moment ${ }^{166}$. Malgré la diminution générale des enceintes boisées des temples et sanctuaires, celles qui ont ainsi été préservées ou récupérées à Meiji sont aujourd'hui bien souvent des sites classés et comptent parmi les derniers espaces urbains ayant conservé des arbres anciens.

Par la suite, avec les fusions des villages, le statut foncier et la répartition des terres des forêts de Wakasugi changèrent plusieurs fois. Mais la forêt domaniale et le périmètre du sanctuaire n'ont pas bougé jusqu'à nos jours. Aujourd'hui, le Taiso-gū est propriétaire d'une totalité de si ha sur la montagne. Dans ceux-ci sont compris l'enceinte des sanctuaires Haut (où se trouve également l'Oku-no-in) et Bas (Gegū 下宮), quelques espaces forestiers sur la montagne et les anciens communaux du mont du Riz (Kome no yama, 22,5 ha) qui étaient anciennement la propriété du quartier de Wakasugi ${ }^{167}$. Indépendamment de cette propriété du sanctuaire et de la

164. Archives Ishii-bō nos 8, 9, I4, III, II8, 527-I, 528, 25, 29.

165. Cf. l'éloge funèbre fait par Ishii Tamaki aux obsèques de Sassa Tamotsu en 1943 (Kokubetsusbiki-ji 告別式辞, daté du 7 février 1943 ; manuscrit conservé par la famille Sassa).

166. Ainsi par exemple, le mouvement de protection des forêts mené Minakata Kumagusu pour les forĉts des sanctuaircs. Voir Torigoc Hiroyuki 鳥越兟之, “ Minakata Kumagusu to Meiji makki no shinrin hogo undō »南方熊楠と明治末期の森林保護運動, Kansai gakuin daigaku shakaigakubu kiyō 関两学院大学社会学部紀要 63 (1991).

167. D'après le registre foncier de Wakasugi, en 1989. 
forêt domaniale (Iooha), les particuliers, le village ( 3 ha de forêts collectives) et la commune de Sasaguri $\left(5 \mathrm{ha}^{168}\right)$ possèdent également des forêts sur le mont Wakasugi. Jusqu’à Meiji, la gestion de la totalité de ces forêts a été assurée par les 70 maisons (ou 6o, selon les époques) du village en accord avec le fief. Originellement, c'était le groupe des maîtres de ces maisons (répartis en 5 sections territoriales), qui, en tant que «siège du sanctuaire» (miyaza 宮座), servait à la fois le Taiso-gũ en assistant le shugen-prêtre pour les rites et la gestion, et entretenait aussi tous les espaces forestiers. C'était alors le pilier central de l'organisation villageoise. En 1923, ces mêmes membres formèrent l'Association pour le développement de Wakasugi (Wakasugi hatten kiseikai 若杉発展期成会), dont le responsable en chef était aussi le maire du village. Effet de l'époque, la fonction de ce conseil fut alors réduite à la seule gestion forestière. Après guerre, en 1954, ce conseil prit le nom de Reihōkai $^{169}$ (《Association du sommet sacré 》 霊峰会), marquant par là, comme l'indiquent ses règlements ${ }^{170}$, sa volonté de renouer avec la fonction historique qui cumulait la gestion des affaires religieuses et forestières. Le Reihō-kai conserva le mode d'accès restrictif limitant ses membres actifs aux chefs des plus anciennes familles du « village d'origine » (bonmura 本村) ${ }^{171}$. En 2010, ils étaient au nombre de 47 (les autres familles ayant quitté le village). Les fonctions actuelles du Reihō-kai sont le travail de gestion et d'entretien (coupes d'éclaircies, détourage, débroussaillage, coupes cycliques, replantation) de la quasi totalité de l'espace forestier du mont Wakasugi, c'est-à-dire la montagne du sanctuaire, la forêt domaniale et la forêt collective de Wakasugi qui sont entièrement plantées en cryptomères (deux tiers) et en cyprès (un tiers) ; l'entretien de l'enceinte du Taiso-gũ ; la gestion de l'oratoire bouddhique de l'Oku-no-in ${ }^{172}$ du sommet (face bouddhique de l'ancienne structure shintō-bouddhique du Taiso-gū) avec ses boutiques de souvenirs et sa buvette, et dont le fonctionnement est toute l'année assuré par des femmes du village qui s'y relaient à tour de rôle. Les revenus du Reihō-kai et de ses membres proviennent

168. Dont une partie est constituée par l'ancienne forêt à bénéfices partagés (bunshürin) entre l'État et 30 particuliers de Wakasugi (datant de 1929), dont les parts ont été rachetées par la commune en 2006 , suite à la chute de la valeur du bois.

169. Association ayant le statut de personnalité juridique.

170. Mentionnés dans les deux premiers alinéas des Règlements du Wakasugi Reibō-kai (Wakasugi reibō-kai kiyaku 若杉霊峰会規約) de 1954, les trois objectifs du Reibō-kai sont d'assurer le "développement du mont Wakasugi-parc régional » et la " promotion de la grandeur du sanctuaire, des temples et lieux saints situés dans cet espace ", ainsi que de "faciliter l'accès aux pèlerins et randonneurs » (Registre du Reibō-kai -I, I954-1973, conservé par le responsable en fonction). Réécrits en 2003 et adaptés au xxI siècle, les Règlements reformulent les objectifs comme étant le «développement des ressources industrielles et touristiques du mont Wakasugi »; et pour ce faire, les membres doivent connaître leurs devoirs et droits concernant «la totalité des biens communautaires et des usages traditionnels de gestion et de société hérités de leurs ancêtres "; et tout en visant l'amélioration de leurs conditions économiques et sociales, ils doivent « renforcer la protection du paysage, des lieux saints et des sites historiques ».

171. Pour les détails de cet historique, voir dans ce même volume, Suzuki Masataka, "Continuités et transformations de la société locale », p. 80.

172. Le service bouddhique de l'Oku-no-in est à la charge quotidienne du temple Kongōchō-in 金剛顶院, temple de Wakasugi fondé en 1926 pour remplacer l'Ishii-bō et situé en dessous du Taiso-gū. 
de toutes ces activités. Une partie est constituée par la rémunération versée par l'Office des forêts pour l'entretien des Ioo ha de forêts domaniales ${ }^{173}$. Si aujourd'hui le Reihō-kai ne s'occupe pas de l'organisation des rites annuels du sanctuaire, il faut néanmoins préciser que les villageois qui ont la charge de ceux-ci, en tant qu'acteurs principaux des rites (dont le prêtre n'est que l'officiant) et membres de l'Association de conservation du kagura 神楽 (danse sacrée) du Taiso ${ }^{174}$, font aussi tous partie de tous ces groupes. Les mêmes personnes remplissent tous ces rôles successivement ou conjointement. Ce sont eux qui, par leur position et dans leurs actes, fédèrent les liens qu'ils entretiennent avec ces différents pôles.

Bien que, suite à la dévalorisation du bois, les activités forestières aient actuellement beaucoup diminué, Wakasugi reste encore un village dont les habitants se considèrent comme des gens de la montagne. Ils pensent et disent que la montagne et ses forêts sont le «bien des membres du Reihō-kai » ${ }^{175}$. Par là, ils mettent l'accent sur le fait que — indépendamment des titres de propriété - cette forêt est pour eux le patrimoine collectif des 47 maisons, noyau identitaire du village, et que ce lien a pour troisième terme le pôle religieux majeur de Wakasugi, dédié aux puissances qui en assurent la protection. Pour eux, entretenir cet espace forestier c'est aussi entretenir la cohésion du village, et vice-versa. La montagne-forêt est ici le point de convergence et d'ancrage des intérêts sociaux, économiques, politiques, culturels et environnementaux, et de toutes les pratiques qui leur sont associées. Tous ces pôles se renforcent et s'éclairent réciproquement. Le sanctuaire, parce qu'il représente aussi une propriété forestière, est à la fois l'enjeu qui concrétise toutes ces tensions, le ressort et le garant de la cohésion de cet ensemble. À ce titre, il est le référent légitimant toutes les démarches et les actes collectifs. Par là on constate que, malgré les « séparations » formelles et les ruptures dans les législations, il y a, aujourd'hui, continuité non seulement de l'imbrication du laïc et du religieux dans lc fonctionnement du groupe villageois qui gère toutes ces structures et activités à la fois, mais aussi - sous une forme contemporaine - de la fusion shintō-bouddhique. Par là, l'exemple de Wakasugi procure un éclairage sur les réalités de ce religieux pluriel, dont une caractéristique me semble être ce que j'ai appelé sa territorialite ${ }^{176}$, c'est-

173. À ceci s'ajoutent une subvention accordée à la forêt ayant le statut de réserve des ressources en eau, et la location de terrains au sommet pour plusieurs antennes de radio et télécommunication.

174. Taiso kagura bozonkai 太祖神楽保存会, association fondée en 1976 pour remplacer l'ancien " siège du kagura »(kaguraza 神楽座), qui détient et transmet les danses rituelles exécutées lors de la fête du sanctuaire et leur accompagnement musical. Les membres en sont tous les aînés des 47 familles actuelles de Wakasugi.

175. Ceci est reflété dans les Règlements du Wakasugi Reibö-kai de 2003 (p. I) : «Ce que ces Règlements appellent bien communautaire (kyōyū zaisan 共有財産), c'est l'ensemble de tous les biens communautaires réels dont le Reihō-kai a hérité de façon coutumière et traditionnelle des époques précédentes ; il en va de même pour les terres et les forêts dont les titres de propriété sont, de façon purement formelle, au nom du sanctuaire et du temple, et qui constituent dans un sens large le patrimoine communautaire. »

176. Anne Bouchy, "Et le culte sera-t-il shintô ou bouddhique ? », Cipango II (2003). Cette territorialisation est bien sûr la forme locale d'un fond de conceptions et d'usages largement partagés par toute la société. 
à-dire son ancrage dans un territoire, un groupe social et ses pratiques. Les uns et les autres se renforçant et se colorant mutuellement. Ici, les forêts de Wakasugi ont directement bénéficié de cette convergence forte, concrétisée par la défense et la gestion collective du sanctuaire Taiso-gū.

Pourtant, plusieurs dangers menacent aujourd'hui cet équilibre. La dévalorisation actuelle du bois provoquant du retard dans les coupes cycliques et la diminution progressive des familles constituant le noyau du village peuvent, à long terme, remettre en question cet équilibre socio-environnemental ancré dans l'histoire. En outre, l'organisation de la gestion autonome de Wakasugi a été bouleversée en 2008. Jusque-là, les 17 maisons du "village originel » (bonmura) avaient continué à constituer un corps administratif indépendant des deux autres entités formées à la suite de la construction, dans la deuxième moitié du $\mathrm{Xx}^{\mathrm{e}}$ siècle, de deux nouveaux quartiers résidentiels dans le bas de Wakasugi (Wakasugi danchi 若杉団地 et Imazato danchi 今里団地), qui possédaient chacun un organisme d'autogestion. Les habitants de ces deux derniers, en tant que nouveaux arrivés étant exclus du groupe des 47 familles placées sous la protection du Taiso-gū (et donc interdits de rôles actifs dans les rites) ainsi que de la gestion des montagnes, constituent en quelque sorte un "dehors du dedans », dont le «village originel » est le «dedans du dedans ». Mais depuis 2008, les normes administratives de l'autonomie locale ont contraint les trois entités à fusionner et à se répartir à tour de rôle les trois fonctions de gestion du quartier (maire, adjoint au maire, agent comptable). Dans les faits, le « village originel » cumule aujourd'hui deux structures. La nouvelle, dans laquelle il n'est qu'une des trois parties constitutives d'un ensemble dont les deux autres composantes détiennent des droits égaux de gestion; et l'ancienne (bonmura) avec son propre maire et ses responsables (comptabilité, organisation économique, gestion de la montagne, des structures religieuses et des rites annuels). La garde de la gestion exclusive du sanctuaire Taiso-gū, de ses rites et danses comme aussi de son enceinte de cryptomères, reste le noyau identitaire et légitimant de la communauté ancienne. Mais, d'une entité forte, unie dans la gestion collective des biens communs, Wakasugi est devenu pluriel. Sa cohésion peut désormais être remise en question, car les deux autres entités, qui représentent une totalité de 200 familles, devraient pouvoir - théoriquement - demander à intervenir, notamment dans les comptes et la gestion des forêts.

L'histoire ancienne et moderne des forêts communautaires de Wakasugi montre une fois encore que, si l'existence de celles-ci repose bien sur la volonté du «dedans » à les défendre, cette volonté se trouve sans cesse en interaction avec les politiques, décisions et apports du dehors qui n'ont cessé de la contrecarrer ou de la renforcer, comme aussi avec les modifications d'un dedans loin d'être statique.

Tous les exemples précédents témoignent de l'existence, à Sasaguri, d'une continuitć dans lcs modalités communautaires et collectives de gestion des espaces forestiers, par-delà tous les bouleversements survenus entre la fin du XIX ${ }^{c}$ et le début du $\mathrm{XXI}^{\mathrm{e}}$ siècle aussi bien dans les systèmes politiques, législatifs, sociaux que dans 
les usages et l'accès aux ressources. On a pu voir que la diversité des modalités de gestion adoptées correspond à la diversité des solutions trouvées pour articuler, de façon viable, les exigences socio-environnementales internes et externes prévalant à un moment donné. Mais, malgré ou à cause de cette diversité, c'est cette continuité collective, ancrée dans la cohésion villageoise, qui a été l'opérateur déterminant dans le jeu constant entre les dynamiques de l'intérieur et de l'extérieur pour la maîtrise des forêts. Ceci semble avoir été la garantie première de la pérennité forestière sur ce territoire communal qui, jusque récemment, a offert, à cet égard, des conditions privilégiées. À l'opposé, la nationalisation et la privatisation des forêts voulues par les gouvernements successifs, ont été objet de méfiance. Car elles sont apparues localement comme une perte de relations entre les communautés villageoises et leurs forêts, et signifiant, à plus ou moins brève échéance, la disparition de cellesci. Lorsqu'elles ont été appliquées, il n'est pas rare, comme on l'a vu, que de façon détournée ou ouverte, le collectif continue à garder sa mainmise sur la gestion réelle des montagnes-forêts. Il ne s'agit pas de survaloriser la gestion traditionnelle des espaces forestiers. L'histoire micro-locale montre bien que cette gestion a sans cesse été tiraillée entre de multiples intérêts et qu'elle a aussi été scandée par des échecs, des catastrophes et des ruptures. Mais cette histoire vient aussi confirmer que la gestion communautaire est bien considérée par une majorité de ses acteurs comme le meilleur garant d'un équilibre des interrelations entre toutes les composantes de cet espace partagé. Tout ceci fait des forêts de Sasaguri un exemple particulièrement riche, non pas de simples oppositions dedans-dehors, mais bien des interactions socio-environnementales multiples, de leurs entrelacs ou « maillage ».

Néanmoins, à la fin du $\mathrm{Xx}^{e}$ et en ce début du $\mathrm{XXI}^{\mathrm{e}}$ siècle, cette continuité a été remise fortement en question, à la fois par les conditions internes (transformations des communautés villageoises) et externes (globalisation des marchés et perte de la valeur du bois, notamment) entraînant des ruptures dans les structures et leur fonctionnement, dans les besoins et les usages. Au niveau des forêts, cela s'est manifesté par l'apparition de phénomènes nouveaux, comme l'abandon des espaces forestiers et de leur entretien. L'« ensauvagement " qui en a résulté a été cause ici comme ailleurs ${ }^{177}$ de lourdes catastrophes socio-environnementales. Les bambouseraies invasives, et les glissements de terrain dans les forêts de conifères non entretenues, en sont les manifestations les plus critiques. Les bambous géants laissés à eux-mêmes détruisent les sols et les chemins, constituent des lieux impénétrables et dangereux à cause des chutes de leurs cannes enchevêtrées. Quant aux arbres abandonnés, ils sont à l'origine de glissements de terrain. L'un des plus récents exemples fut celui qui eut lieu à Yamate en 2009, suite au typhon $\mathrm{n}^{\circ} 6$ (du 16 au 21 juillet) qui a provoqué des chutes de pluies torrentielles entre le 24 et le 29 juillet ( $560 \mathrm{~mm}$ en trois jours, record de pluviométrie à ce jour). Des coulées de boue ont dévalé des sommets jusqu'au bas des pentes des montagnes situées à l'est de Yamate. Elles provenaient des plantations des forêts domaniales qui ne sont ni entretenues

177. John Knight, "When Timber Grows Wild: The Desocialisation of Japanese Mountain Forests ", dans Philippe Descola \& Gísli Pálson (éd.), Nature and Society. Anthropological Perspectives, 1996. 


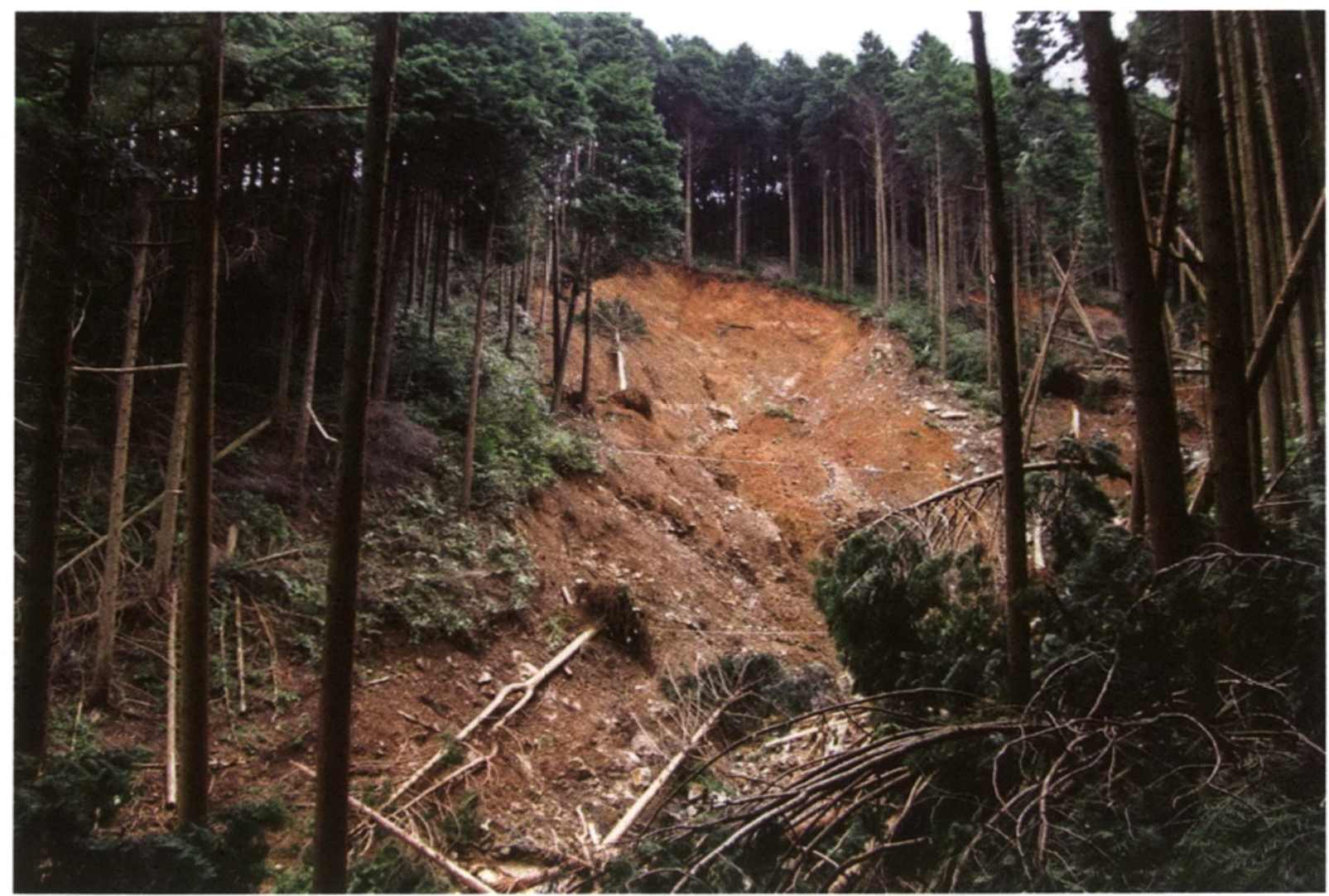

Fig. 12 : Point initial du glissement de terrain lors du typhon des 16-2x juillet 2009 : plantations de cyprès aux racines peu profondes (Yamate).

ni coupées depuis la dévalorisation du bois. Les racines très superficielles de conifères devenus beaucoup trop grands n'ont pu retenir le poids des arbres qui ont été arrachés, libérant des tonnes de boue mêlée de troncs et de rochers qui ont tout détruit sur leur passage (fig. I2 et 13). Ceci fut la cause de deux décès et de dégâts matériels considérables (destruction de routes et ponts, bâtiments emportés, etc.). L'invasion des bambous repose principalement sur le délaissement des terres par les habitants. Le non-entretien des forêts est attribué à la mauvaise gestion de l'Office national et régional des forêts.

Face à cette situation qui ne fait qu'empirer, s'est manifestée récemment une réaction collective positive pour l'environnement végétal. Depuis 2008 une politique de gestion qui s'est fixée des objectifs concrets a été mise en place par la commune, soutenue par l'aide financière, logistique et humaine des habitants. Parmi ces objectifs, certains sont déjà en cours de réalisation : la conservation des plus anciennes forêts de cryptomères du mont Wakasugi (sur le sommet et autour du sanctuaire) et leur classement en tant que patrimoine culturel pour l'ancienneté de leur plantation ; l'arrachage de toutes les bambouseraies ${ }^{178}$ pour en replanter l'espace en feuillus (des

178. Les bambous sont réduits en poudre et celle-ci est utilisée comme engrais dans les rizières ou épandue sur les chemins. 


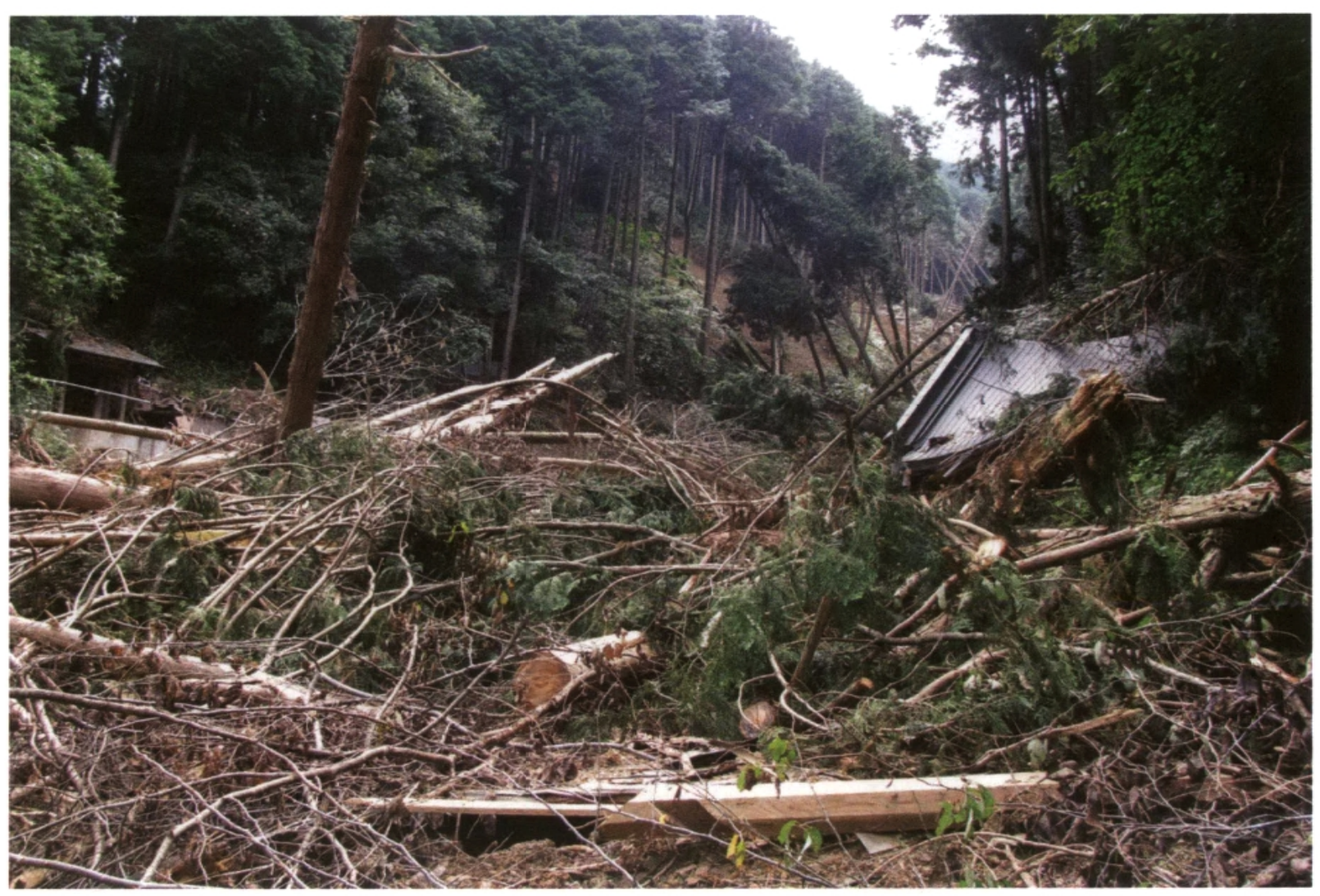

Fig. I3 : Après le passage de la vague de boue, de roches et d'eau : les cyprès renversés (Yamate, au dessus du temple Ichi-no-taki-ji) (août 2009).

dons ont été faits par les riverains, notamment par les temples du circuit de pèlerinage des 88 lieux saints, dont une partie des chemins envahie par les bambous était devenue impraticable); grâce à l'impôt départemental sur les forêts ${ }^{179}$, l'entretien, la conservation voire le rachat des forêts plantées par des particuliers qui ne s'en occupent plus, ainsi que l'acquisition (par rachat ou cession par les descendants) des terres appartenant à des particuliers dans les montagnes-forêts (notamment dans les fonds de Kido) dont les propriétaires ont disparu, et leur replantation en feuillus ; des battues aux sangliers - attirés par l'ensauvagement forestier et ravageant les espaces cultivés - assurées régulièrement par l'association des chasseurs. Ces mesures sont un retour à la prise en charge collective des forêts, non plus par les anciens villages, mais sous la forme élargie de la commune. Mais la plus originale de toutes ces entreprises reste la « thérapie forestière " évoquée au tout début de ce travail.

179. Depuis 2008, le département de Fukuoka prélève un impôt sur les forêts (shinrin kankyōzei 森林環境税) de 500 yens par contribuable imposé en tant qu'agriculteur. Il est reversé à chaque commune concernée qui utilise ces fonds pour la gestion des forêts. 
Le label de thérapie forestière (Shinrin Serapī 森林セラピー) a été obtenu par la commune de Sasaguri en mars 2009 auprès de la Société de thérapie forestière ${ }^{180}$, organisme qui dirige ce programme forestier à l'échelle nationale. Le coût - élevé - de l'enregistrement est de cinq millions de yens. Celui-ci donne droit à l'utilisation du label et du sigle (fig. 14) sur toutes les plaquettes publicitaires et brochures de promotion de Sasaguri, ainsi que sur les cartes de visite des élus. La commune fut la $36^{\mathrm{c}}$ du pays et la $4^{\mathrm{c}}$ de Kyūshū à accéder à cette labellisation, qui, en 2013, a été accordée à un total de 53 municipalités. Comme ailleurs, l'objectif de la municipalité est d'abord d'attirer les visiteurs en espérant des retombées économiques, et de trouver par ce biais de quoi soutenir sa nouvelle politique de protection des forêts. Mais la particularité de ce qui s'est passé ici à la suite de cette initiative communale est la façon dont l'ensemble des parties concernées dans Sasaguri a saisi cette occasion, d'une part, pour se regrouper autour du projet et, d'autre part, pour en faire l'instrument de redynamisation de divers éléments existants mais qui étaient en perte de vitesse ou en voie de disparition. Dans la mouvance de l'éco-tourisme et du tourisme vert, des circuits de loisir, de tourisme, de santé avaient été mis en place plus ou moins récemment. Mais ils n'ont pas bénéficié d'une telle mobilisation. Autour de la « thérapie forestière " se sont regroupés pour agir, non seulement les structures d'hébergement, de restauration et les entreprises municipales, mais aussi les temples du « nouveau pèlerinage de Shikoku » dont les fidèles sont en baisse régulière depuis un certain temps, ou encore les autres structures et organisations religieuses locales, des groupes religieux de l'extérieur, et plus généralement les habitants. En effet, outre la qualité et le déploiement de ses espaces forestiers, Sasaguri bénéficie de l'existence de circuits déjà présents (le parcours des 88 temples) avec leurs infrastructures (notamment des toilettes publiques). Les parcours pédestres de la «thérapie forestière » réutilisent ces chemins, en grande partie abandonnés depuis l'essor de la pratique du pèlerinage en automobile. Ils ont ainsi pu être remis en état, ce qui fut aussi l'occasion de nettoyer les espaces forestiers qu'ils traversent.

180. Shinrin Serapī sosaeti 森林セラピーソサエティ, société fondée en 2008, qui regroupe, sous l'égide de l'Office des forêts (ministère de l'Agriculture et des Forêts), des offices gouvernementaux (dont l'Organisation pour la promotion du reverdissement du territoire national, Kokudo ryokka suishin kikō 国土緑化推進機構), des organismes de recherche et des entreprises (<http://www. fo-society.jp/> [date du dernier accès 21/o5/2013]). Elle a remplacé le Groupe de recherche sur la thérapie forestière, formé en 2004 , suite à la reconnaissance par les pouvoirs publics des effets bienfaisants des forêts sur la santé humaine, démontrés par les chercheurs dès les années 1980 . Initialement, c'est sous le nom de «bains de forêt " (shinrin yoku) que ce contact bénéfique avec les forêts fut promu auprès du grand public. L'accent mis sur l'aspect scientifique et thérapeutique démarque ses actions de celles d'organismes qui s'appuient sur le seul argument de l'environnement. L'objectif est de combiner la préservation de l'environnement forestier en augmentant les sites labellisés (selon un ensemble de critères vérifiés régulièrement) et d'y développer un ensemble de pratiques (marche, repos, gymnastique et autres) bénéfiques pour la santé et libératrices du stress pour les citadins. Les conditions de labellisation impliquent la qualité des structures d'accueil, la facilité de l'accès pour les citadins, la possibilité d'organiser des circuits dans les forêts, la qualité des arbres et de leur entretien, etc., le tout étant analysé régulièrement à partir de critères physiologiques, psychologiques et physiques mesurables. Le mot-clé est iyashi 㴓 L, " apaisement, cure, guérison ». Les municipalités ayant reçu cet agrément proposent des sites et des chemins. 


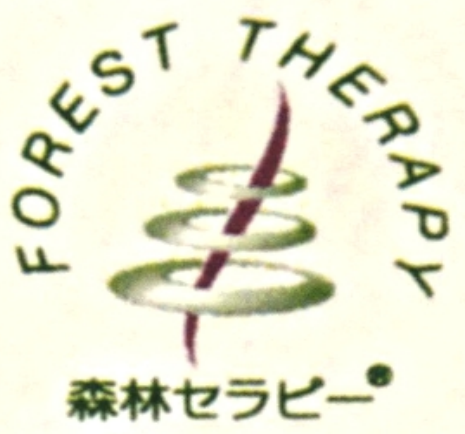

Fig. 14 : Sigle de la thérapie forestière.

C'est-à-dire aussi de mettre à contribution les communautés des anciens villages sur le territoire desquels ces sentiers passent. Les responsables des 88 temples et oratoires, dont les bâtiments se trouvent, pour une majeure partie d'entre eux, au cœur ou en bordure des forêts, ouvrent leurs espaces aux visiteurs voulant se reposer, ou pratiquer méditation et yoga. Ceci est l'occasion d'engager un nouveau type de dialogue et de relations de part et d'autre. On assiste ainsi au renouvellement de la formule quelque peu dépassée de l'ancien pèlerinage des 88 temples, par le biais d'initiatives internes et externes, adaptées à la demande actuelle, mais utilisant toutes les facilités des structures antérieures. Et cette réalisation, en retour, a permis à certains supérieurs de temples de créer des formes différentes de pèlerinage dans et hors de la commune. Pour l'hébergement, les lieux d'accueil des temples, ainsi que 2 I auberges et hôtels situés dans les montagnes sont partie prenante du projet. La thérapie forestière est ainsi à l'origine de multiples effets interactionnels.

Les circuits de thérapie forestière avec guides et programmes d'activités diverses ont été ouverts au public en avril 2010. Or c'est l'ensemble des chemins de Sasaguri qui a en fait bénéficié de cette initiative. Aujourd'hui, 13 parcours pédestres ( $90,125 \mathrm{~km}$ balisés et entretenus), dont quatre portant le label de thérapie forestière, sont proposés aux randonneurs comme la possibilité d'un « rafraîchissement du corps et de l'esprit dans la verdure " (fig. I5). Si les premiers s'étendent sur toute la commune en joignant tous les lieux du pèlerinage et traversant ses parcs et espaces verts aménagés ${ }^{181}$, les quatre parcours de thérapie forestière (représentant une totalité de $25,95 \mathrm{~km}$ ) sont tous situés dans les forêts de cryptomères sur les pentes du mont Wakasugi, et aboutissent à son sommet, c'est-à-dire au sanctuaire Taiso-gū. Ce dernier, avec l'Oku-no-in où jaillit la source sacrée, a été considéré de longue date comme l'Oku-no-in (le point d'aboutissement et de référence) général pour tout le pèlerinage du nouveau Shikoku à Sasaguri. Or, avec la thérapie forestière, il a pris une dimension supplémentaire, bien vue par le Reihō-kai qui gère ses structures d'accueil. 52 candidats ont répondu à la demande de la commune pour une formation de guides ${ }^{182}$, alors qu'on n'en espérait qu'une trentaine. On compte aujourd'hui 29 guides ( 14 femmes et 15 hommes entre 30 et 60 ans), qui vivent à Sasaguri, mais dont la majorité est originaire d'autres localités. Toute l'année, ils organisent deux ou trois excursions de thérapie forestière par mois à participation payante. Les prix varient selon les prestations ajoutées à la marche (repas, hébergement, enseignement de yoga, marche nordique, etc.). Selon les dates, sont programmées des activités entrant dans le cadre religieux (visite de temple, participation à des rites religieux, rencontre avec un supérieur, méditation, ascèse de la cascade, transcription de

I8I. Ci-dessus II, I P. I47-I49.

182. Ils doivent acquérir l'accréditation de "guide " ou de « thérapeute forestier ». 


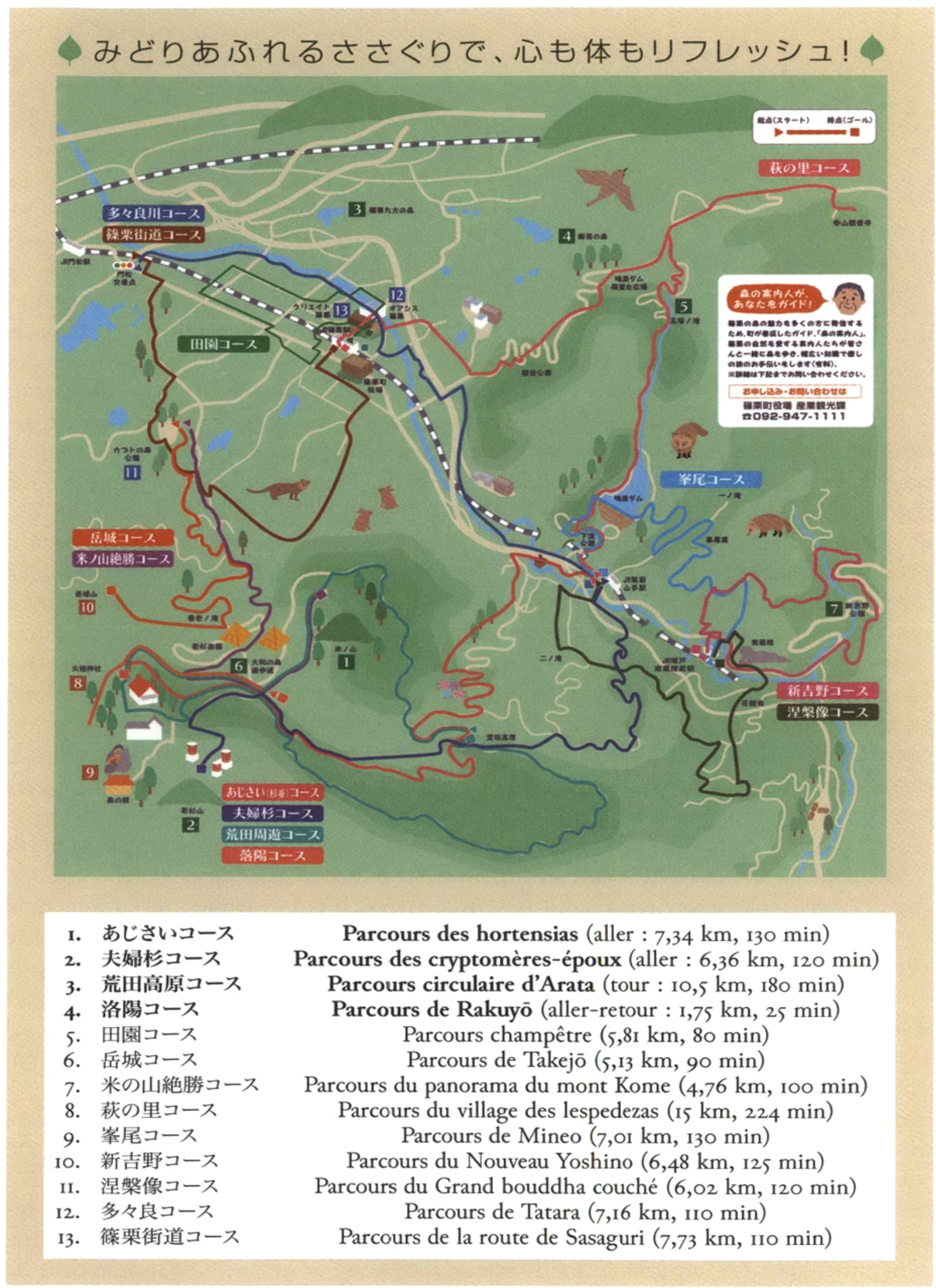

Fig. 15 : Les 13 parcours pédestres de Sasaguri, dont 4 sont des chemins de thérapie forestière. 
sūtra, etc.). Les guides suivent une formation supplémentaire auprès des temples afin de maîtriser les pratiques de pèlerinage et de culte particulières à Sasaguri ${ }^{183}$. Le site internet de la commune héberge une page de thérapie forestière très richement documentée sur ses circuits, ses programmes, et propose des formulaires d'inscription à ces excursions ${ }^{184}$. On y trouve des comptes rendus sous forme de journaux personnels et des tweets envoyés par les participants, majoritairement des citadins (principalement des femmes au foyer d'une cinquantaine d'années et de jeunes employés ou sportifs), qui viennent de la région environnante, et surtout de Fukuoka. Leur attente combine une volonté de (re-)découvrir la « nature » et leur propre corps avec ses ressources sensibles et spirituelles, et celle d'acquérir, dans ce but, des connaissances sur les plantes, leurs usages thérapeutiques et culinaires, et plus globalement un ensemble de savoirs traditionnels sur le vivant et le vécu. Cette demande correspond à celle qui est exprimée par toute une frange importante de la société qui, manquant de modèles et s'étant détournée des modalités religieuses traditionnelles, cherche des ouvertures, tant vers les nouveaux mouvements spirituels que vers tout autre moyen nouveau, pour accéder à une sagesse de la vie passant par le contact avec le monde naturel et l'expérience intime. Â cet égard, les circuits de Sasaguri, avec l'enchâssement des sites religieux dans ses forêts et les prestations proposées, offrent un « plus » qui dépasse le seul registre végétal.

On peut voir ainsi dans la thérapie forestière expérimentée à Sasaguri, à la fois une nouvelle forme d'un religieux diffus associée à des modalités renouvelées des pratiques de pèlerinage, et aussi la plus récente formulation de la relation communautaire aux forêts dans ce territoire communal. Nouvelle appropriation de l'espace coutumier par le biais d'une demande du dehors articulée aux besoins de la commune, cette initiative a conduit les gens du dedans à voir et gérer encore autrement les milieux dans lesquels ils vivent. Tant par la diversité de ses acteurs internes et externes que par les multiples dimensions - naturelles, culturelles, économiques, administratives, politiques, logistiques - qui s'y déploient, cette relation éclaire bien l'entrelacs complexe qui préside ici à la destinée des espaces forestiers, aujourd'hui comme autrefois. La demande extérieure joue un rôle déterminant dans la revalorisation, voire dans la pérennité, d'un patrimoine local naturel et culturel acculé au vieillissement. Il est intéressant de constater le poids des valeurs extérieures, " universellement " reconnues, comme la science et la médicalisation de l'expérience, dans cet engouement réactivé pour les forêts de Sasaguri. L'un des arguments fondamentaux étant la preuve scientifique de leur efficacité « thérapeutique " à réduire le taux de cortisol, hormone de stress, et améliorer les réactions du système nerveux sympathique. On peut y déceler sans doute la forme la plus actuelle de la socialisation de ces forêts, qui continuent ainsi à être un milieu hautement anthropisé.

Par-delà les ruptures d'époque et de ton, les habitants de Sasaguri montrent ici leur capacité à renouveler les formules anciennes, en créant des modalités de gestion

183. Voir dans ce volume Nakayama Kazuhisa, «La dynamique de création, réplication et déclin des lieux de pèlerinage ", p. 284-287.

184. Sasaguri-chō sbinrin serapī kicbi 簙栗町森林セラピー基地 <http://www.sasaguri-therapy. $\mathrm{jp} />$ [date du dernier accès 21.5.2013]. 
dans lesquelles communauté micro-locale et collectivité élargie jusqu'à l'extérieur du territoire communal collaborent pour entretenir, par là, la continuité dans les relations aux forêts et dans les représentations qu'ils en ont. En effet, tout ce qui précède montre bien que la perception des forêts et son expression actuelle reflètent, aujourd'hui comme hier, une ambiguïté dans la définition des notions (modernes) de «culture » et de « nature " qui s'y trouvent étroitement imbriquées. À l'inverse, une telle nature culturalisée, mais vue et donnée comme éminemment "naturelle », s'oppose à ce qui est déclaré sauvage. L'embroussaillement n'est pas nécessairement un état « sauvage ", car il peut être temporaire et maitrisé (jachères, retour cyclique des bois et broussailles sur brûlis, etc.). Ce qui est « sauvage », c'est l'espace dont la gestion n'est pas ou plus assurée par un groupe social, et ainsi que l'on ne connaît plus parce que l'on ne s'y rend pas. Â ce titre, à Sasaguri, que pourtant elles recouvrent sans grands interstices sur plus de la moitié du territoire, les forêts ne représentent pas l'élément "sauvage ». Si elles le sont devenues en certains lieux, on a vu qu'il s'agit d'un phénomène récent, redouté de tous, car cause de catastrophes. Les forêts ensauvagées ont pour origine le délaissement, dû à la perte de maîtrise de gestion par ceux qui en sont les riverains immédiats et donc les premiers concernés par leur devenir (à la suite soit du morcellement entre des particuliers, soit de l'accaparement par l'État). Prises en charge par le village ou d'autres formes contemporaines de collectivité locale complexe ou élargie, les montagnes-forêts entrent au contraire dans la catégorie du territoire et du végétal socialisés. Ainsi leur gestion communautaire ou collective impliquant les gens du lieu s'avère être et avoir été le meilleur garant de leur protection et de leur entretien. Car une telle gestion prend en compte les risques et les bénéfices réels de l'exploitation des forêts par rapport à l'ensemble des facettes de la vie locale. Ceci empêche que les espaces forestiers ne soient considérés comme un bien dont chacun pourrait disposer à sa guise, et contribue au contraire à ce qu'ils soient considérés et gérés comme l'environnement par excellence de la vie sociale, auquel on se sent non seulement matériellement mais aussi socialement et moralement lié. C'est sur cet arrière-plan que les forêts sont déclarées à la fois « nature » et « patrimoine culturel».

Le choix du cryptomère du mont Wakasugi comme arbre-symbole de la commune de Sasaguri et de ses milieux « naturels » est ainsi hautement significatif. Ce n'est pas la laurisylve de Kana.ide, les forêts auto-générées les plus anciennes de la commune, qui ont été érigées en signe représentatif de la communauté locale, mais bien le cryptomère, dont l'existence à cet endroit est, en fait, témoignage d'« artificialité ». Comme on l'a vu précédemment, ce choix s'appuie sur deux éléments qui définissent la présence même de cet arbre comme due à l'intervention humaine : le cryptomère ne peut exister sur ces montagnes que s'il est planté par bouturage; et la référence première avancée en est la légende fondatrice éponyme du mont Wakasugi, citée dans toutes les présentations et brochures de Sasaguri : la transplantation par l'impératrice Jingū d'une branche de l'aya-sugi, arbre sacré du sanctuaire de Kashii (Fukuoka), au retour de sa victoire sur les Trois royaumes de Corée. La valorisation repose ici sur le jeu dehors-dedans qui intègre la micro-histoirc dans l'histoire (mythique) nationale. D’où le nom de " parcours pédestre du Yamato » (Yamato no mori yūho-dō 大和の森遊歩道) au milieu des cryptomères du mont Wakasugi, 
qui évoque à la fois le Japon, la lignée impériale, la haute Antiquité. Or cette valorisation hyperbolique de l'ancienneté du processus de culturalisation de la nature et des arbres eux-mêmes sert à mettre en avant le naturel de la forêt. On voit ici par quels cheminements, reliant le passé et le futur dans les réalisations présentes, la montagne-forêt culturalisée et socialisée est pensée et donnée à penser comme étant la « nature ». Et c'est ainsi qu'elle est proposée aux citadins à la recherche des bienfaits de la «thérapie forestière ». 


\section{Bibliographie}

\section{AкIMICHI Tomoya 秋道智彌}

2010 Komonzu no chikyūshi. Gurōbalu-ka jidai no kyōyūron ni mukete コモンズ の地球史一一グローバル化時代の共有論に向けて, Tōkyō, Iwanami Shoten 岩波書店.

ARIOKA Toshiyuki 有岡利幸

2004 Sato-yama II 里山 2, coll. «Mono to ningen no bunkashi » II8, vol. 2, Tōkyō, Hōsei-daigaku shuppan-kyoku.

BERKES, F. et al.

1989 "The Benefits of the Commons ", Nature 340, p. 91-93.

1990 "The Tragedy of the Commons: Twenty-Two Years Later », Human Ecology i8, vol. I, p. I-I9.

Bouchy, Anne

2000 «La cascade et l'écritoire - Dynamique de l'histoire du fait religieux et de l'ethnologie du Japon : le cas du shugendô — ", BEFEO 87, vol. I, p. $34 \mathrm{I}-366$.

2003 «Et le culte sera-t-il shintô ou bouddhique ?", Cipango II, p. 45-98.

2006 «De l'ethnologie du Japon : par qui, où, comment ? », Ateliers 30, p. 63-99.

2012 "'Initiatic landscape' and Shugendō mountain-entry ", Proceedings 148, Londres, The Japan Society, p. 73-99.

Bourdier, Marc \& Pelletier, Philippe (éd.)

2000 L'archipel accaparé. La question foncière au Japon, coll. «Série Études japonaises ", Paris, EHESS, vol. 3.

\section{ChiBA Tokuji 千葉徳爾}

1973 Hage yama no bunka はげ山の文化, coll. «Nihon no rekishi chiri 》日本の 歴史地理 4, Tōkyō, Gakuseisha 学生社, vol. 4 .

\section{FujIMURA Miho 藤村美穂}

2001 “"Minna no mono” to wa nanika »みんなのもの」とは何か, dans Ino.ue Makoto 井上真, Miya.uchi Taisuke 宮内泰介 (éd.), Komonzu no sbakaigaku. Mori, kawa, umi no sbigen kyōdō kanri wo kangaeru コモンズの社会学——森・ 川・海の資源共同管理を考える, Shirizu Kankyō shakaigaku シリーズ環境社 会学 2, Tōkyō, Shin.yōsha 新曜社, vol. 2, p. 32-54.

Furushima Toshio 南島敏雄 (éd.)

1955 Nibon rin.ya seido no kenkyū 日本林野制度の研究, Tōkyō, Tōkyō daigaku shuppankai 東京大学出版会.

\section{Hardin, Garrett}

1968 "The Tragedy of the Commons », Science 162, p. 1243-1248.

\section{HiroKawa Yūji 廣川鿆司}

2012 «Kankyō hozen ni kiyo suru "sōyūteki shoyūkan" ni yoru kōkyōteki tochi riyō chitsujo no keisei » 噮境保全に笴与する「総有的所有観」による公共的土 地利用秩序の形成, Kökyō kenkyū 公共研究 8, vol. I, p. 130-170. 
Houdart, Sophie

2002a « L'image ou sa dissolution au moment de la préparation de l'Exposition internationale japonaise de 2005 ", Autrepart 24 p. 141-166.

2002b « Paysages et jardins : un autre regard " Japon, hiver 2001-2002, Ebisu 28, p. 243-254.

2003 «Exposer la nature d'hier, construire la nature de demain. L'Exposition internationale japonaise de 2005 ", $1^{\text {er }}$ Congrès du Réseau Asie, 2003, Thématique v, Atelier 14, L'Exposition internationale japonaise de 2005 », I $^{\text {er }}$ Congrès du Réseau Asie, 2003. <http://www.reseau-asie.com/colloque/ thematiques-du-Ier-congres-2003/atelier-14-representation-du-milieunaturel/> [dernier accès le 17 octobre 2014]

2004 «Le sato-yama comme arène politique au moment de la préparation de l'Exposition internationale japonaise, Aichi 2005 ", Japon pluriel, Actes du colloque de la SFEJ, p. 259-269.

2006 " 'Un chaos savamment ordonné...' L'élaboration conceptuelle de l'Exposition internationale japonaise de 2005 ", Ateliers 30, p. $35^{-62}$.

INGOLD, Tim

2000 The Perception of the Environment: Essays on Livelibood, Dwelling and Skill, Londres, Routledge.

2007 Lines: A Brief History, Londres et New York, Routledge.

2011 Being Alive: Essays on Movement, Knowledge and Description, Londres, Routledge.

INO.UE Makoto 井上真, MiYA.UCHI Taisuke 宮内泰介 (éd.)

2001 Komonzu no shakaigaku. Mori, kawa, umi no shigen kyōdō kanri wo kangaeru コモンズの社会学——森・川・海の資源共同管理を考える, Shirīzu Kankyō shakaigakuシリーズ環境社会学 2, Tōkyō, Shin.yōsha 新曜社, vol. 2.

JEAN, Florence

2001 La propriété arboraire en Corse et dans les pays environnants d'Europe et du pourtour de la Méditerranée, thèse de droit, Corte.

KADA Yukiko 嘉田由紀子

1997 《Seikatsu jissen kara tsumugi dasareru jūsōteki shoyūkan 》生活実践か らつむぎ出される重層的所有観, Kankyō sbakaigaku kenkyū 環境社会学研究, $\mathrm{n}^{\circ}$ 3, p. $72-85$.

2001 «Gyorō to kankyō hozen. Biwako no sesshō kindan to gyogyōken wo meguru shinsei no rekishi kara saguru 》漁労と環境保全——琵琶湖の殺 生禁断と漁業権をめぐる心性の歴史から探る, dans Torigoe Hiroyuki 鳥越晧之 (éd.), Sbizen kankyō to kankyō bunka 自然瓄境亡環境文化, Kōza Kankyō shakaigaku 請座:環境社会学 3, Tōkyō, Yūhikaku, 有斐閣, vol. 3, p. 47-76.

KNIGHT, John

1996 «When Timber Grows Wild: The Desocialisation of Japanese Mountain Forests », dans Philippe Descola \& Gísli Pálson (éd.), Nature and Society. Anthropological Perspectives, Londres et New York, Routledge, p. 221-239. 
Kondō Tenji 近藤典二

1993 《Fukuokahan no sanrin ni tsuite 》福岡藩の山林について, Fukuokaken chiikishi kenkyū 福岡県地域史研究 $\mathbf{I}$, p. 78-III.

LEFEBVRE, Henri

2000 La production de l'espace, Paris, Anthropos [ $\mathrm{I}^{\mathrm{e}}$ éd. 1974].

LeroI-Gourhan, André

2004 Pages oubliées sur le Japon, Grenoble, Jérôme Millon.

Matsushrta Koji

2012 «Recent Problems and New Directions for Forest Producer Cooperatives Established in Common Forest in Japan ", dans Diez J. Julio (éd.), Sustainable Forest Management. Case Studies, Rijekia (Croatia), InTech, p. 162-I82.

Matsushita Koji \& Hirata Kunihito

2002 "Forest Owners' Associations », dans Iwai Yoshiya (éd.), Forestry and the Forest Industry in Japan, Vancouver, UBC Press, p. 41-66.

Mitsumata Gaku 三俣学

2006 "Shichōson gappei to kyūson zaisan ni kansuru ichi kōsatsu. Kankyō hozen-komyuniti saikō no jidai no shichōson gappei no giron ni mukete » 市町村合併と旧村財産に関する一考察——環境保全・コミュニティ再考の時代の 市町村合併の議論にむけて, Nibon minzokugaku 日本民俗学 245 (numéro spécial de la revue des études d'ethnologie du Japon consacré à la fusion des communes), p. 68-98.

MiYajima Hiroshi 宮島寛

1994 Nibon no tennen sugi 日本の天然スギ, texte non publié de la conférence au Symposium "Yakushima de katarō “Nihon no sugi” " 屋久島で語ろう:日 本の柇 (26 novembre 1994), Yaku-chōritsu Yaku-sugi shizen-kan 屋久町 立屋久杉自然館.

MIYA.UCHI Taisuke 宮内泰介

2001 "Komonzu no shakaigaku. Shizen kankyō no shoyū, riyō, kanri wo megutte 》コモンズの社会学——自然環境の所有・利用·管理をめぐって, dans Torigoe Hiroyuki 鳥越皓之 (éd.), Shizen kankyō to kankyō bunka 自然環境 と環境文化, Kōza Kankyō shakaigaku 講座:環境社会学 3, Tōkyō, Yūhikaku, 有斐閣, vol. 3, p. $25-46$.

MiYA.UCHI Taisuke 宮内泰介 (éd.)

2006 Komonzu wo sasaeru shikumi コモンズをささえるしくみ, Tōkyō, 新翟社. Shin. yōsha.

Moreno, Diego, Hordynsky-Caillat, Lada, Redon, Odile, Serventi, Silvano 1989 "Châtaigneraie "historique" et châtaigneraie "traditionnelle". Notes pour l'identification d'une pratique culturale », Médiévales 16, vol. 17, p. I47-161.

MORI Hiroko 森弘子

2008 Hōman-zan no kankyō rekishi gaku-teki kenkyū 宝満【小環境歴史学的研究， Tōkyō, Iwata shoin 岩出書院. 
NAKamura Hisashi 中村尚司, et al. (éd.)

1995 Komonzu no umi コモンズの海, Tōkyō, Gakuyō shobō 学晹書房.

NisHida Yoshikazu 西田彦..

2009 Iri.ai rin.ya to shüben shakai 入会林野と風辺社会, Kyōto, Nakanishiya shuppan ナカニシヤ出版.

Nomoto Kan.ichi 野本寛一

1987 Seitai minzokugaku josetsu 生態民俗学序説, Tōkyō, Hakusuisha 日水社.

Nomoto Kan.ichi 野本寛一, FukuTA Ajio 福田アジオ et al., éd.

1996 Kankyō no minzoku 罟境()民俗, Koza Nihon minzokugaku 满坐:呠本の民 俗学 4, Tōkyō, Yūzankaku shuppan 雄山閣出版, vol. 4 .

OKUCHI Shō 奥地正

1974 《Nihon kokuyūrin ni okeru rōdō soshiki no keisei to tenkai »国有林に おける労働組織の形成と展開 I, Ritsumeiknan keizaigaku 许命館経済学 23, vol. 4 , p. 447-489.

ORIKUCHI Shinobu 折口信夫

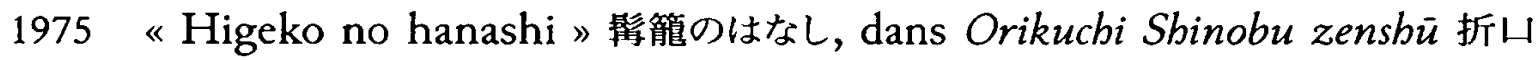
信大全集 2, Tōkyō, Chūō kōronsha 中央公論社, vol. 2, p. 182-2II [ I re éd. I915].

PÉREZ, Patrick

2012 «Elements of an American Landscape: The Arizona Hopi », dans Arnar Àrnason et al. (éd.), Landscapes beyond Land: New Etbnographies of Landscape and Environment, New York, Berghahn Books, p. 83-97.

2013 "Ce que les Hopi m’ont appris sur le paysage », Les Annales de Géograpbie 69ı, p. $243^{-265}$.

SASAKI Kōmei 佐々木高明

1971 Inasaku izen 稲作以前, coll. «NHK Books », Tōkyō, Nihon hōsō shuppan kyōkai 日本放送出版協会.

1982 Shōyojurin bunka no michi 照葉樹林文化の道, coll. « NHK Books », Tōkyō, Nihon hōsō shuppan kyōkai 日本放送出版協会.

2009 Nibon bunka no tayōsei 日本文化の多様性, Tōkyō, Shogakkan 小学館.

SASAKI Tetsuya 佐々木哲哉

2007 No no kioku. Hito to kurashi no genzō 野の記憶一一人暮らしの原像, Fukuoka, Genshobō 弦書房.

SHIDEI Tsunahide 四手井綱英

1985 Shinrin 森林, coll. « Mono to ningen no bunkashi »ものと人間の文化史 53, vol. I, Tōkyō, Hōsei-daigaku shuppan-kyoku 法政大学出版局.

1998 Sbinrin II 森林 II, coll. «Mono to ningen no bunkashi » 53, vol. 2, Tōkyō, Hōsei-daigaku shuppan-kyoku.

2000 Shinrin III 森林 III, coll. «Mono to ningen no bunkashi » 53, vol. 3, Tōkyō, Hōsei-daigaku shuppan-kyoku. 
2006 Shinrin wa mori ya hayasbi dewanai. Watashi no shinrinron 森林はモリや八 ヤシではない——私の森林論, Kyōto, Nakanishiya shuppan ナカニシヤ出版.

SHINOHARA Tōru 篠原徹

1990 Sbizen to minzoku 自然と民俗, Tōkyō, Nihon Editāsukūru shuppan-bu 日本エディタースクール出版部.

SugA Yutaka 营豊

2001 "Shizen. Shizen wo meguru minzoku kenkyū no mittsu no chōryū » 自然:自然をめぐる民俗研究の三つの潮流, Nibon minzokugaku 日本民俗学 227, p. $14-29$.

2005 《Komonzu to seitōsei »コモンズと证当性, Kankyō shakaigaku kenkyū 環境 社会学研究 2, p. 22-39.

2006 Kawa wa dare no mono ka. Hito to kankyō no minzoku-gaku 川は誰のも のか一一人と環境の民俗学, Tōkyō, Yoshikawa Kōbunkan 吉川弘文館.

TORIGOE Hiroyuki 鳥越皓之

1997 《Komonzu no riyōken wo kyōju suru mono »コモンズの利用権を字受す る者, Kankyō sbakaigaku kenkyū 環境社会学研究 3, p. 5-14.

1991 《Minakata Kumagusu to Meiji makki no shinrin hogo undō 》南方熊楠 と明治末期の森林保謢運動, Kansai gakuin daigaku sbakai-gakubu kiyō 関四 学院大学社会学部紀要 63, p. 277-297.

TORIgoe Hiroyuki 鳥越皓之 (éd.)

1994 Kokoromi toshite no kankyō minzokugaku 試みとしての環境民俗学, Tōkyō, Yūzankaku shuppan.

TsuboI Hirofumi 坪井洋文

1979 Imo to Nibonjin イモと日本人, Tōkyō, Miraisha 未来社.

1982 Ine wo eranda Nibonji 稻を選んだ日本人, Tōkyō, Miraisha 未来社.

YANO Tatsuo 矢野達雄

2007 《Shichōson gappei to iri.ai rin.ya »市町村合併と入会林野, dans Miyazaki Yoshirō 宮崎幹朗 (éd.), Ebime-ken ni okeru shichōson gappei no tenkai to tenbō 愛媛県における市町村合併の展開と展望, Matsuyama 松山 (Ehime-ken 愛媛県), Ehime daigaku bungaku-bu sōgō seisaku gakka 愛媛大学文学部総 合政策研究学科, p. 247-266 (peut être consulté aussi à <http://nsi.shudo-u. ac.jp/-tyano/gappei \%20to\%2orinya.pdfs [date du dernier accès 08/02/2013]).

Monographies, historiographies locales et documents

AOYAGI Tanenobu 青柳種信 (comp.)

1993 Cbikuzen no kuni zoku fudoki sbūi 筑前国続風土記拾遺, Tōkyō, Bunken shuppan 文献出版 ( $\mathrm{I}^{\mathrm{e}}$ éd. $1814-64$ ).

Buraku-yū rin.ya tōitsu ni kansuru keii 部落有林野統一に関する経緯 (date non indiquée mais estimée vers 1930) communiqué par la mairie de Sasaguri.

Chikushino shishi hensan iinkai 筑紫野市史編さん委員会 (éd.)

1999 Chikusbino sbisbi 筑紫野书史民俗編, Chikushino-shi 筑紫野市 (Fukuoka-ken). 
Cbikuzen Kasuyagun Wakasugi Taiso jinja engi 筑前糟屋郡分杉大祖神社緣起 [Genroku 元禄 5, 1692]. Kyūshū rekishi shiryōkan 九州歴史資料館, éd., Chikuzen Kasuya. Wakasugi yama no bukkyô iseki 筑前粕屋 若杉山の仏教遺跡, coll. Kyūshū no jisha shirīu 九州の寺社シリーズ 8, Dazaifu 太宰府, Kyūshū rekishi shiryōkan, I986, vol. 8, p. 38-44.

Gōya Takeshiro 合屋武城

1957 Cbikuzen Wakasugi kyōdoshi 筑前若杉郷土誌, Sasaguri, éd. privée.

Hacbiman Usagū On-takusensbū 八幡宇佐宮御䚾宣集 (XII ${ }^{\mathrm{e}}$ siècle) Shigematsu Akihisa 重松明久, éd., 1986. Hachiman Usagū On-takusenshū 八幡宇佐宮 御託宣集, Tōkyō, Gendai shinchōsha 現代思潮社.

KaIBARA Ekiken 貝原益軒 (comp.)

1977 Cbikuzen no kuni zoku fudoki 筑前国続風土記, Fukuoka-ken shi shiryō zoku dai yon shū. Chishi ben $I$ 福岡県史資料続第四輯: 地誌編I, Tōkyō, Bunken shuppan 文献出版 (comp. 1688-1710).

Kasuya-gun yakusho 糟屋郡役所 (éd.)

1972 Kasuyagun-shi 糟屋郡志, Tōkyō, Meicho shuppan 名著出版 ( ${ }^{\mathrm{e}}$ éd. I924).

KIYOHARA Dabutsurō 清原陀佛郎 (éd.)

1935 Tō Kinsaku ō 藤金作翁, Fukuoka (hors commerce).

Kurose Shigefumi 黒瀬茂文

2009 Wakasugi yama osugi bunpu cbōsa, daigoji, 若杉山大スギ分布調查, 第5次, Sasaguri, Sasaguri-chō kyōiku-iinkai 篠栗町教育委具会.

KYūsHū rekishi shiryōkan 九州歴史資料館 (éd.)

1986 Cbikuzen Kasuya. Wakasugi yama no bukkyō iseki 筑前粕屋 若杉山の仏教 遺跡, coll. Kyūshū no jisha shirīzyu 九州の寺社シリーズ 8, Dazaifu 人草拊.

Minami.ISHI Takeshi 南石武 (éd.)

1968 Kasuya yōroku 粕屋要録, Fukuoka, Shiseki hozon dōkōkai 史跡保存同好会.

Nishi Nihon Bunka kyōkai 西日本文化協会 (éd.)

1988 Fukuoka-ken chiri zensbi 福岡県地理全誌 I, Kindai shiryō-ben 近代史料編 de Fukuoka-ken-shi 福岡県史 22, Fukuoka, Fukuoka-ken 福岡県, vol. 6, 22.

Nōrin suisan-shō 農林水産省 (Ministry of Agriculture, Forestry, and Fisheries)

2012 Annual Report on Forest and Forestry, Tōkyō, Ministry of Agriculture, Forestry, and Fisheries (peut être consulté aussi à <http://www.rinya. maff.go.jp/j/kikaku/hakusyo/24hakusyo/pdf/h24summary.pdf $>$ [date du dernier accès 28/os/2012]).

Sasaguri-chō bunkazai senmon-iinkai 篠栗町文化財專門委員会 (éd.)

1982 Sasaguri chōshi. Rekishi-ben 篠栗町誌:歷史編, Sasaguri, Sasaguri-chō yakuba 篠栗町役場 (abrégé en Sasaguri chōshi).

1990 Sasaguri cbōshi. Minzoku-ben 篠栗町誌:民俗編, Sasaguri, Sasaguri-chö yakuba 策栗町役場. 
Sasaguri-chō kikaku kōhōka 篠栗町企画広報課 (éd.)

2003 Green Oasis Sasaguri. 2003 Dai niji Sasaguri-cbō kokudo riyō keikaku 第:. 次篠栗町国土利用計画, Sasaguri, Sasaguri-chō yakuba 篠栗町役場.

2006 Sasaguri-chō. Chōsei yōran, shiryōben 策栗町:町势要覧・資料編, Sasaguri, Sasaguri-chō 篠栗町.

2008 Green Oasis Sasaguri, 2008-2012 Dai yoji Sasaguri-cbō sōgō keikaku. Kōki kibon keikaku 第 4 次篠栗町総合計画, 後期基本計画, Sasaguri, Sasaguri-chō yakuba 篠栗町役場.

Sasaguri-chō 篠栗町 (éd.)

1964 Sasaguri. Chōsei yōran ささぐり:町勢要覧, Sasaguri, Sasaguri-chō 篠栗町.

TAKAHASHI Katsubee 高橋勝兵衛 (éd.)

1908 Kasuyagun Seto-son sonze, kan 糟屋郡勢門村々是・完 (Archives du Centre de documents historiques et matériaux ethnographiques, Sasaguri rekishi minzoku shiryō shitsu 篠栗歴史民俗資料室).

Tō Kihachirō 藤喜八郎 (éd.)

1907 Kasuyagun Sasaguri-son sonze, kan 糟屋郡策栗村々是・完 (Archives du Centre de documents historiques et matériaux ethnographiques, Sasaguri rekishi minzoku shiryō shitsu 篗栗歴史民俗資料室).

Wakasugi reibō-kai kiyaku 若杉霊峰会規約 (Règlements du Wakasugi Reibō-kai), 1954. Wakasugi reibō-kai kiyaku 若杉霊峰会規約 (Règlements du Wakasugi Reibō-kai), 2003.

Ressources internet

Fukuoka-kenchō 福岡県庁 (site officiel du département de Fukuoka). <http://www. pref.fukuoka.lg.jp> [date du dernier accès 03/05/2012].

Kokusei chōsa 国勢調査 (recensements nationaux) [2007] (site de l'Agence des statistiques du ministère de l'Intérieur, Sōmu-shō tōkei-kyoku 総務省統 計局). <http://www.stat.go.jp/data/nenkan/indexi.htm> [date du dernier accès $11 / 08 / 2012]$.

Rin.yachō 林野行 (site officiel de l'Office de la sylviculture). <http://www.rinya. maff.go.jp> [date du dernier accès II /08/20I2].

Rin.yachō 林野宁, Heisei 24 nendo sbinrin ringyō bakusbo平成 24 年度 森林・林業白書. <http://www.rinya.maff.go.jp/j/kikaku/hakusyo/24hakusyo> [date du dernier accès 28/os/2012].

Sasaguri-chō shinrin serapi kichi 篠栗町森林セラピー基地. <http://www.sasaguri-therapy. $\mathrm{jp} />$ [date du dernier accès 21/05/2013].

Shinrin serapi sosaeti 森林セラピーソサエティ<http://www.fo-society.jp/> [date du dernier accès 21/05/2013].

Shinrin sbigen no genkyō 森林資源の現況, 2007, site du Rin.yachō (site officiel de l'Office de la sylviculture). <http://www.rinya.maff.go.jp/j/keikaku/ genkyou/hrg/2_i.html> [date du dernier accès II/08/2012]. 
Sōmushō 総務省, 《7-22 todōfuken, shoyū keitai-betsu genkyō sbinrin menseki » 都道 府県, 所有形態別現況森林面積.<http://www.stat.go.jp/data/nihon/zuhyou/ no702200.xls> [date du dernier accès 25/05/2013]. 


\section{Résumé en japonais/H本語要旨}

外部の政策と内部の戦略の間に摇れる地域共同体と森林空間の関係性-D—篠柴の川林

当共同研究計画の一賟として、本稿では、篠栗町の地域社会が町面程の 60 \%以上を占めている 山林空間といかに向き合い、相互に関係してきたかという問題に焦点をあてて考察した。この相互関 係のありようがこれまでどのように形成され、また現在もどのように形成され続けているかを検討するこ とで、筆者は、地域共闰体の「内」と「外」の次元を形作っては壊してゆく国家や国際的な政治・社会、 経済の変動という文脈の中で、自然環境や地域共同体の変遷を支配してきた複雑な力学を明らかに することを試みる。それによって、篠栗の森林がどのような意味で、そして、なにゅえに、「自然」であると 同時に「文化遺産」であると言えるのかを、よりよく理解することが可能になる。

このために、筆者は、はじめに篠栗奵の行政区域をこの地域の地理的・社会的な全休像のなかに 仿置づけ、の地域がさまざまな交通路によっていかに外部に開かれているかを示す。またこの論考 の位置づけを明らかにするため、日本の民俗学や社会人類学の概念や方法論に関する背景を簡単 に説明する。また篠栗の自然・植物環境の多様性を示した上で、第2部では、政策や近年の経済危 機によって何度も激変してきた民俗資源としての「山林」の使用法についてまとめ、所有権制度の変 化による構造的な影響を明らかにする。こで問題となっているのは、まず管理と利用のための共同 体の装置であり、また物質的・文化的かつ社会的な「遺涯」としての「里山」に対する共同体の関係 でもあり、さらに、より公い意味でこれらの森林空閒が形成する環境そのものでもある。第3部では、 森林組合や複数の行政的・社会的組織体の間で形成されている「分収森」などの森林の共同運営 のあり方の諸夕イプを分析する。この考察は、人会地」(コモンズ)の近代における変遷や、それらの 今日的な再評価に関して、地域社会の文脈だけではなく、人文科学の文脈からも光を当てることに も結びつくことになる。最後に、若杉村落の事例を分析することによって、これらすべての小学を一つ に結びつけ、さらに森林に対する地域共同体の関係のなかで決定的な役㓶を果すオペレーターと しての宗教性を、諸問題のなかに組み入れることが可能になる。篠栗町が 2009 年に獲得した「森 林セラピー」の国家認証は、地域のアイデンティテイ形成の中心となる森林との関わり方に関する、 最も新しい様式であるように思われる。 